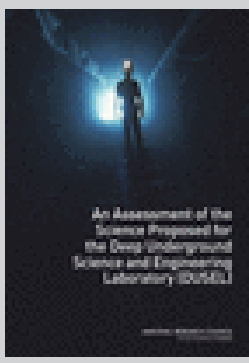

\title{
An Assessment of the Deep Underground Science and Engineering Laboratory
}

ISBN

978-0-309-21723-1

94 pages

$7 \times 10$

PAPERBACK (2011)
Ad Hoc Committee to Assess the Science Proposed for a Deep Underground Science and Engineering Laboratory (DUSEL); National Research Council

\section{Visit the National Academies Press online and register for...}

Instant access to free PDF downloads of titles from the

NATIONAL ACADEMY OF SCIENCES

- NATIONAL ACADEMY OF ENGINEERING

INSTITUTE OF MEDICINE

nATIONAL RESEARCH COUNCIL

$10 \%$ off print titles

Custom notification of new releases in your field of interest

Special offers and discounts

Distribution, posting, or copying of this PDF is strictly prohibited without written permission of the National Academies Press. Unless otherwise indicated, all materials in this PDF are copyrighted by the National Academy of Sciences.

Request reprint permission for this book 


\section{An Assessment of the Science Proposed for the Deep Underground Science and Engineering Laboratory (DUSEL)}

Ad Hoc Committee to Assess the Science Proposed for a Deep Underground Science and Engineering Laboratory (DUSEL)

Board on Physics and Astronomy

Division on Engineering and Physical Sciences

NATIONAL RESEARCH COUNCIL

OF THE NATIONAL ACADEMIES

THE NATIONAL ACADEMIES PRESS

Washington, D.C.

www.nap.edu 


\section{THE NATIONAL ACADEMIES PRESS 500 Fifth Street, N.W. Washington, DC 20001}

NOTICE: The project that is the subject of this report was approved by the Governing Board of the National Research Council, whose members are drawn from the councils of the National Academy of Sciences, the National Academy of Engineering, and the Institute of Medicine. The members of the committee responsible for the report were chosen for their special competences and with regard for appropriate balance.

This study was supported by Grant No. PHY1016162 between the National Academy of Sciences and the National Science Foundation and by Grant No. DE-SE00004240 between the National Academy of Sciences and the U.S. Department of Energy. Any opinions, findings, conclusions, or recommendations expressed in this publication are those of the author(s) and do not necessarily reflect the views of the organizations or agencies that provided support for the project.

International Standard Book Number-13: 978-0-309-21723-1

International Standard Book Number-10: 0-309-21723-7

Additional copies of this report are available from the National Academies Press, 500 Fifth Street, N.W., Lockbox 285, Washington, DC 20055; (800) 624-6242 or (202) 334-3313 (in the Washington metropolitan area); Internet, http://www.nap.edu; and the Board on Physics and Astronomy, National Research Council, 500 Fifth Street, N.W., Washington, DC 20001; Internet, http://www.nationalacademies.org/bpa.

Copyright 2012 by the National Academy of Sciences. All rights reserved.

Printed in the United States of America 


\section{THE NATIONAL ACADEMIES Advisers to the Nation on Science, Engineering, and Medicine}

The National Academy of Sciences is a private, nonprofit, self-perpetuating society of distinguished scholars engaged in scientific and engineering research, dedicated to the furtherance of science and technology and to their use for the general welfare. Upon the authority of the charter granted to it by the Congress in 1863, the Academy has a mandate that requires it to advise the federal government on scientific and technical matters. Dr. Ralph J. Cicerone is president of the National Academy of Sciences.

The National Academy of Engineering was established in 1964, under the charter of the National Academy of Sciences, as a parallel organization of outstanding engineers. It is autonomous in its administration and in the selection of its members, sharing with the National Academy of Sciences the responsibility for advising the federal government. The National Academy of Engineering also sponsors engineering programs aimed at meeting national needs, encourages education and research, and recognizes the superior achievements of engineers. Dr. Charles M. Vest is president of the National Academy of Engineering.

The Institute of Medicine was established in 1970 by the National Academy of Sciences to secure the services of eminent members of appropriate professions in the examination of policy matters pertaining to the health of the public. The Institute acts under the responsibility given to the National Academy of Sciences by its congressional charter to be an adviser to the federal government and, upon its own initiative, to identify issues of medical care, research, and education. Dr. Harvey V. Fineberg is president of the Institute of Medicine.

The National Research Council was organized by the National Academy of Sciences in 1916 to associate the broad community of science and technology with the Academy's purposes of furthering knowledge and advising the federal government. Functioning in accordance with general policies determined by the Academy, the Council has become the principal operating agency of both the National Academy of Sciences and the National Academy of Engineering in providing services to the government, the public, and the scientific and engineering communities. The Council is administered jointly by both Academies and the Institute of Medicine. Dr. Ralph J. Cicerone and Dr. Charles M. Vest are chair and vice chair, respectively, of the National Research Council. 
Copyright (C) National Academy of Sciences. All rights reserved. 


\title{
COMMITTEE TO ASSESS THE SCIENCE PROPOSED FOR A DEEP UNDERGROUND SCIENCE AND ENGINEERING LABORATORY
}

\author{
ANDREW J. LANKFORD, University of California at Irvine, Chair \\ YORAM ALHASSID, Yale University \\ EUGENIO COCCIA, University of Rome "Tor Vergata” \\ CHARLES FAIRHURST, Itasca Consulting Group, Inc. \\ BRADLEY W. FILIPPONE, California Institute of Technology \\ PETER FISHER, Massachusetts Institute of Technology \\ TAKAAKI KAJITA, University of Tokyo \\ STEPHEN E. LAUBACH, University of Texas at Austin \\ ANN NELSON, University of Washington \\ RENE A. ONG, University of California at Los Angeles \\ FRANK J. SCIULLI, Columbia University \\ MARJORIE SHAPIRO, University of California at Berkeley and E.O. Lawrence \\ Berkeley National Laboratory \\ JAMES M. TIEDJE, Michigan State University \\ DAVID WARK, Imperial College London
}

Staff

DONALD C. SHAPERO, Director

JAMES C. LANCASTER, Program Officer

CARYN J. KNUTSEN, Associate Program Officer TERI G. THOROWGOOD, Administrative Coordinator BETH DOLAN, Financial Associate 


\section{BOARD ON PHYSICS AND ASTRONOMY}

ADAM S. BURROWS, Princeton University, Chair

PHILIP H. BUCKSBAUM, Stanford University, Vice Chair

RICCARDO BETTI, University of Rochester

JAMES DRAKE, University of Maryland

JAMES EISENSTEIN, California Institute of Technology

DEBRA ELMEGREEN, Vassar College

PAUL FLEURY, Yale University

PETER F. GREEN, University of Michigan

LAURA H. GREENE, University of Illinois at Urbana-Champaign

MARTHA P. HAYNES, Cornell University

JOSEPH HEZIR, EOP Group, Inc.

MARC A. KASTNER, Massachusetts Institute of Technology

MARK B. KETCHEN, IBM Thomas J. Watson Research Center

JOSEPH LYKKEN, Fermi National Accelerator Laboratory

PIERRE MEYSTRE, University of Arizona

HOMER A. NEAL, University of Michigan

MONICA OLVERA DE LA CRUZ, Northwestern University

JOSE N. ONUCHIC, University of California at San Diego

LISA J. RANDALL, Harvard University

MICHAEL S. TURNER, University of Chicago

MICHAEL C.F. WIESCHER, University of Notre Dame

Staff

DONALD C. SHAPERO, Director

ROBERT L. RIEMER, Senior Program Officer

JAMES C. LANCASTER, Program Officer

DAVID B. LANG, Program Officer

CARYN J. KNUTSEN, Associate Program Officer

TERI G. THOROWGOOD, Administrative Coordinator

BETH DOLAN, Financial Associate 


\section{Preface}

The size, complexity, and costs of the instruments of scientific inquiry are almost as diverse as the questions addressed in those inquiries. They range from atomic physics experiments that rest on a tabletop and might be assembled for less than a quarter of a million dollars, to accelerator complexes that spread over tens of square kilometers and cost billions of dollars to build. Underground laboratories are a relatively recent addition to this array of facilities. Built to shield extremely sensitive detectors from the noise of their surrounds and the signals associated with cosmic rays, underground facilities have been established over the last 30 years at a number of sites worldwide. To date, the United States' efforts to develop such facilities have been modest and consist primarily of a small underground laboratory in Soudan, Minnesota, and the Sanford Underground Laboratory, a developmental research laboratory at the site of the proposed Deep Underground Science and Engineering Laboratory (DUSEL) in Lead, South Dakota. Researchers from the United States who are interested in pursuing experiments that require these ultraquiet spaces have typically worked in collaboration with others in large underground facilities built in Europe, Japan, and Canada.

Over the past 50 years, the U.S. underground science community, principally researchers in nuclear and particle physics, has pushed for a larger underground laboratory on the scale of the major facilities located in other countries. This push gained significant support during the long-range planning process of the nuclear and physics communities. The nuclear physics community placed the building of an underground facility as a top priority for its field, and the particle physics community recognized the importance of such a facility for achieving several of 
the scientific goals in its field. ${ }^{1}$ Proceeding with the development of a major underground research facility was also encouraged and supported through workshops and advisory committees sponsored by the Department of Energy (DOE) and the National Science Foundation (NSF). Consequently, the NSF, working with DOE and the science community, formally evaluated the science that might take place at such a facility, developed an initial suite of experiments, and then selected a potential site for those experiments, an abandoned mine in Lead, South Dakota (the "DUSEL facility").

During the final deliberations on whether to proceed with the program, DOE and NSF approached the National Research Council (NRC) and requested that it provide an independent assessment of the science possibilities associated with construction of the DUSEL facility and how the program proposed for the facility would impact both the stewardship of the research communities involved and broader public interests. The statement of task for the committee that was assembled to carry out this effort - the Committee to Assess the Science Proposed for the Deep Underground Science and Engineering Laboratory (DUSEL) — is as follows:

The committee will undertake an assessment of the proposed DUSEL program, including:

- An assessment of the major physics questions that could be addressed with the proposed DUSEL and associated physics experiments,

- An assessment of the impact of the DUSEL infrastructure on research in fields other than physics,

- An assessment of the impact of the proposed program on the stewardship of the research communities involved,

- An assessment of the need to develop such a program in the U.S., in the context of similar science programs in other regions of the world,

- An assessment of broader impacts of such an activity, including but not limited to education and outreach to the public.

Shortly before the committee's first meeting in mid-December 2010, the National Science Board (NSB), which is the governing board of the NSF, decided not to provide an interim \$29 million for bridge funding to support the further development of the DUSEL facility until it would enter its final design stage in the summer of 2011. As described in the FY2012 budget request for NSF's Directorate for Mathematical and Physical Science submitted several months later, the decision not to provide the bridge funding was part of a larger determination by the NSB that because the scope and likely cost of the project were outside of NSF's core mission responsibilities, NSF would not be the principal steward of the DUSEL

${ }^{1}$ DOE/NSF. 2007. The Frontiers of Nuclear Science: A Long Range Plan. Report of the Nuclear Science Advisory Committee, p. 7; DOE/NSF. 2008. US Particle Physics: Scientific Opportunities: A Strategic Plan for the Next Ten Years, Report of the Particle Physics Project Prioritization Panel, p. 2. 
facility. ${ }^{2}$ From the time of that December 2010 decision through the time this report was written, DOE and NSF have been discussing whether to proceed with some or all of the program described in the material developed for the DUSEL project. Those discussions notwithstanding, the path that will be taken for developing an integrated underground research facility, or even whether such a facility will be built, has not been decided.

The NSB's decision not to proceed as steward of the program had an immediate impact on the approach that this committee needed to take in addressing its charge. Obviously, the "proposed DUSEL program" referred to in the statement of task did not have the same meaning after the NSB's decision as it had had before. For any underground laboratory, the facility itself is part of the experiment, and much of the science that can be pursued depends on the specifics of that facility-among them, how deep it is; how large its experimental chambers are; how structurally sound it is; and how far it is from other facilities with which it intends to jointly pursue research. Before NSB's December 2010 decision, a specific suite of experiments was to take place at specific underground locations in a specific site for the committee to evaluate. Since that decision, the scope of the DUSEL program has become much more amorphous. Nonetheless, for purposes of this report, the committee chose to treat the slate of experiments described to it at the first meeting as the "proposed DUSEL program."3 Furthermore, it understands that those experiments will be included in a preliminary design report to be submitted to the NSF by the project's principal investigator. Although the committee understands that the DUSEL program envisioned when this committee was assembled is not proceeding, the science to be accomplished in the principal physics experiments proposed for that program remains compelling and argues for the value of developing a U.S. facility, not necessarily on the scale of the originally proposed DUSEL, where these crucial experiments can be conducted and resources shared.

As a final note, at the committee's first meeting, representatives of the NSF asked the committee to address issues such as whether the NSF-DOE partnership, as it then existed, is necessary and is appropriately structured and whether the DUSEL program merits the investment required to accomplish it. Because these issues are only tangentially, if at all, related to the science questions to be addressed, the committee respectfully declined to make these judgments. ${ }^{4}$

\footnotetext{
${ }^{2}$ Available at http://www.nsf.gov/about/budget/fy2012/pdf/fy2012_rollup.pdf, page Overview-9. Last accessed on July 6, 2011.

${ }^{3}$ See Statement of Task, Appendix A.

${ }^{4}$ As pointed out in a recent NRC study, Assessment of Impediments to Interagency Collaboration on Space and Earth Science Missions (Washington, D.C.: National Academy Press, 2010), deciding whether or not to pursue a multiagency collaboration and, then, if one chooses to proceed, how to do so effectively, raises many difficult issues and challenges. That report provides guidance for addressing those issues.
} 
At its first two meetings, the committee heard from a number of people who are either formally or informally involved in helping to develop the DUSEL facility and program. The committee is grateful to these individuals for their information and insights; the materials they provided were a valuable resource for the committee's work. Finally, I am particularly grateful to the members of this committee and to the staff who worked diligently on a demanding schedule to produce this report.

Andrew Lankford, Chair

Committee to Assess the Science Proposed for the Deep

Underground Science and Engineering Laboratory (DUSEL) 


\section{Acknowledgment of Reviewers}

This report has been reviewed in draft form by individuals chosen for their diverse perspectives and technical expertise, in accordance with procedures approved by the National Research Council's (NRC's) Report Review Committee. The purpose of this independent review is to provide candid and critical comments that will assist the institution in making its published report as sound as possible and to ensure that the report meets institutional standards for objectivity, evidence, and responsiveness to the study charge. The review comments and draft manuscript remain confidential to protect the integrity of the deliberative process. We wish to thank the following individuals for their review of this report:

Lisa Alvarez-Cohen, University of California at Berkeley,

Frank Calaprice, Princeton University,

Francis Halzen, University of Wisconsin at Madison,

Wick Haxton, University of California at Berkeley,

Ernest Henley, University of Washington,

Joseph Hezir, EOP Group, Inc.,

Mark Peters, Argonne National Laboratory,

John Schiffer, Argonne National Laboratory, and

Yannis Yortsos, University of Southern California

Although the reviewers listed above have provided many constructive comments and suggestions, they were not asked to endorse the conclusions or 
recommendations, nor did they see the final draft of the report before its release. The review of this report was overseen by Julia Phillips, Sandia National Laboratories, as monitor, and James Brau, University of Oregon, as coordinator. Appointed by the NRC, they were responsible for making certain that an independent examination of this report was carried out in accordance with institutional procedures and that all review comments were carefully considered. Responsibility for the final content of this report rests entirely with the authoring committee and the institution. 


\section{Contents}

1 OVERVIEW

Study Background, 5

The DUSEL Program, 5

This Study, 7

Science Overview, 9

Physics Experiments, 9

Nonphysics Experiments, 14

Broader Impacts, 15

Co-location of Experiments, 15

National Facility, 16

2 DESCRIPTION OF UNDERGROUND FACILITIES

General Characteristics of Underground Laboratories, 19

Survey of Selected Laboratories, 21

Deep Underground Science and Engineering Laboratory/Sanford Underground Laboratory (United States), 22

Soudan Underground Laboratory (United States), 23

Sudbury Neutrino Observation Laboratory (Canada), 24

Laboratori Nazionali del Gran Sasso (Italy), 24

Kamioka Observatory (Japan), 25

China Deep Underground Science and Engineering

Laboratory (China), 26 
3 SCIENCE ASSESSMENTS

Physics Program, 29

Dark Matter, 29

Tests of Grand Unification Theories, 38

Neutrino Physics Overview, 41

The Nature of Neutrinos-Oscillations (Long-Baseline

Neutrino Experiment), 43

The Nature of Neutrinos-Antiparticles, Mass Scale

(Neutrinoless Double-Beta Decay), 51

Proton Decay, 58

Nuclear Astrophysics, 63

Neutrino Astrophysics, 69

Nonphysics Science and Engineering Programs, 72

Overview, 72

Subsurface Engineering Challenges, 74

Geoscience Challenges, 76

Bioscience Challenges, 79

Limitations, 80

Experimental Details, 81

Potential Future Lines of Inquiry, 86

4 IMPACTS OF A NATIONAL UNDERGROUND FACILITY

Shared Infrastructure and Integrated Oversight, 88

Stewardship for the Research Communities, 90

Need to Develop Such a Program in the United States, 95

General Considerations, 95

Specific Considerations, 97

Summary of the Need, 100

Broader Impacts, 101

Visibility of the U.S. Scientific Accomplishment, 101

Educational and Outreach Opportunities, 102

\section{APPENDIXES}

$\begin{array}{llr}\text { A } & \text { STATEMENT OF TASK } & 107\end{array}$

$\begin{array}{ll}\text { B MEETING AGENDAS } & 108\end{array}$

C BIOGRAPHIES OF COMMITTEE MEMBERS 112

D SURVEY OF THE PRINCIPAL UNDERGROUND LABORATORIES 118 


\section{Summary}

Underground laboratories are a relatively new kind of research facility, developed primarily because they provide the extremely quiet environment needed to study rare events such as proton decay and the faint signals associated with neutrinos-ghostly particles with very little mass and no net charge that only weakly engage with most "normal" matter. As weak or rare as those signals are, their study will have profound implications; breakthroughs in any of the leading physics experiments that study these signals will be the foundations upon which a significant portion of the physics community builds for decades to come.

Because of the importance of these studies, a number of underground research facilities have been built around the world, including a modest facility in the United States. Led by the National Science Foundation (NSF) and working in conjunction with the Department of Energy (DOE), the research communities that engage in underground science in the United States developed an integrated research program centered around a major underground facility to be located in South Dakota: the Deep Underground Science and Engineering Laboratory (DUSEL). As part of the process of developing DUSEL and the program associated with it, NSF and DOE jointly commissioned this study. The principal charge to the committee was to independently assess the physics questions that could be addressed with the proposed program, how such a program would impact the stewardship of the research communities involved, and whether there was a need to develop such a program in the United States, given similar science programs elsewhere. The committee also was charged with assessing the potential impact of this facility on research in nonphysics fields and on broader interests such as education and public outreach. 
In response to this charge, the committee concludes that three of the proposed physics experiments-(1) a direct detection dark matter experiment on a scale of one to tens of tons, (2) a long-baseline neutrino oscillation experiment, and (3) a ton-scale, neutrinoless double-beta decay experiment-are of paramount and comparable scientific importance. Each of these experiments addresses at least one crucial question upon which the tenets of our understanding of the Universe depend. A direct detection dark matter experiment (1) would seek to learn the nature of the mysterious dark matter that makes up approximately 80 percent of the material Universe, a subject of enormous significance to astrophysics and particle physics. A long-baseline neutrino oscillation experiment (2) would significantly advance the study of neutrino properties, particularly if it is coupled with a neutrino beam produced using a new high-intensity proton source at Fermilab. It would also provide increased sensitivity for the possible detection of proton decay and neutrinos from supernovas, phenomena whose observation would be momentous for science. A neutrinoless double-beta decay experiment (3) could determine whether neutrinos are their own antiparticles, the answer to which will help us understand how the Universe has evolved. Each of the three experiments is the central component of an ongoing scientific program and could result in a breakthrough discovery upon which particle physics, nuclear physics, and astrophysics will build. The committee concludes that exceptional opportunities will result from proceeding with plans to build in the United States a world-leading long-baseline neutrino experiment and developing within the United States both one direct dark matter detection experiment on the ton to multiton scale and one neutrinoless double-beta decay experiment on the ton scale for installation at a U.S. site or, if such a site is not available, at an appropriate overseas facility. Pursuing this program would not only allow us to address scientific questions of paramount importance but, as discussed below, would also have a significant positive impact on the stewardship of the particle and nuclear physics research communities and would result in the United States assuming a visible leadership role in the expanding field of underground science.

The neutrino oscillation experiment (2) would be a significant improvement over existing experiments in another respect as well: its sensitivity to the detection of proton decay, another consequential physics experiment that has been proposed for DUSEL. The stability of the proton is a crucial issue that will provide a direct window onto the grand unification of forces and the origin of matter. Nonetheless, while the added potential of the experiment would be welcome, the ability to search for evidence of proton decay should not be the primary factor in selecting the neutrino detector technology or in siting the experiment.

The neutrino oscillation detector (2) also would contribute to the study of supernovas, one of the most important astrophysical phenomena. These are sufficiently rare occurrences - approximately two per century within our galaxy — that 
it is possible none will occur during the long lifetime of the experiment. However, the information gained by studying such an event with the detectors under consideration for DUSEL would give us enormous insight into events that are essential in galaxy formation and in the determination of the elemental composition of solar systems such as ours. The committee concludes that the ability to study these rare events adds great value to the neutrino oscillation experiment but should not be a significant consideration in choosing the neutrino detector technology or siting.

The committee found, moreover, that a fourth physics experiment, a nuclear astrophysics study to measure low-energy nuclear cross sections relevant to astrophysical processes, would be scientifically important. These cross sections are quite small, and efforts to measure them need the protected environment provided by underground laboratory space to filter out competing signals. Construction of a small underground accelerator facility would enable these scientifically important measurements.

The proposed DUSEL facility would provide unique opportunities for fields outside of physics — the geosciences and subsurface engineering — to explore in situ the physical and mechanical properties of rock at depths and over areas and times not currently available to them. Among the proposed experiments are regulated studies of the influence of fracture systems on rock response to applied loads and of the interdependence of the thermo-hydromechanical-chemical-biologic aspects of subsurface systems, and efforts to make rock more "transparent" by developing imaging techniques that would allow the exploration of subsurface material at a distance despite its opacity. Enabling the geoscience and subsurface engineering fields to conduct such studies would be an important step forward for these fields. The subsurface environment would also give biology researchers an opportunity to explore life in extreme environments and to learn how biological systems manage to live in the conditions that exist deep underground.

Co-locating the three main underground physics experiments at a single site would allow infrastructure, personnel, and expertise to be shared. Co-location would also contribute to stewardship by fostering synergy among the communities and by offering an existing infrastructure for future experiments, either extensions of the original research program or new research initiatives. By developing a facility where these experiments are co-located, the United States would be seen as a leader in the expanding field of underground science. Lastly, the existence of such a facility would allow the above-mentioned small underground accelerator facility for studying processes of nuclear astrophysics to benefit from the shared infrastructure, personnel, and expertise.

In light of the valuable experiments in subsurface engineering, the geosciences, and biosciences that could be enabled by an underground research facility, the committee recommends the development of a mechanism to allow scientists 
in fields other than physics to perform research at an underground physics facility in the United States.

Finally, the report assesses how access to a national facility for underground research would advance the current set of studies and also provide opportunities for future studies. The committee concludes that such a facility would be of longterm benefit to a substantial portion of the physics community and other scientific communities and that it would guarantee the United States a leadership role in the expanding global field of underground science generally and on the "intensity frontier" of the particle physics community in particular. 


\section{1 \\ Overview}

According to the big bang theory, our Universe began in a state of unimaginably high energy and density, contained in a space of subatomic dimensions. At that time, unlike today, the fundamental forces of nature were presumably unified and the particles present were interacting at energies not attainable by present-day accelerators. The features of the Universe we observe today, like the large-scale distribution of luminous and dark matter and the preponderance of matter over antimatter, resulted from the behaviour of unknown elementary particles in that primordial epoch. The physics of this earliest of states can be assessed through the search for certain spontaneous but very rare phenomena in matter and through the detection of the weak effects of highly elusive particles. Underground laboratories provide the conditions needed to investigate these processes and have succeeded in discovering the first clear evidence for physics beyond the Standard Model: namely, that those extremely elusive particles_-neutrinos-are massive and that flavor lepton numbers are not conserved. These laboratories now appear to be the gateway to understanding the physics of the grand unification of the forces of nature.

\section{STUDY BACKGROUND}

\section{The DUSEL Program}

Within the confines of the existing underground mines and laboratories in the United States and abroad, the U.S. research community has always played a leading 
role in underground science. Examples of landmark experiments in this country include the first observation of solar neutrino oscillations by the Brookhaven solar neutrino experiment begun in the 1960s in the Homestake mine in Lead, South Dakota; ${ }^{1}$ the first limits imposed by the Grand Unification Theory (GUT) on proton decay at the Irvine-Michigan-Brookhaven experiment in a Morton salt mine; ${ }^{2}$ and the pioneering solid-state direct-detection dark matter experiment, Cryogenic Dark Matter Search, in the Soudan mine. ${ }^{3}$ As the required sensitivity and scale of underground experiments grow, the need for new underground laboratory space has drawn the attention and proposals of research communities around the world (see Chapter 2). The U.S. particle and nuclear physics communities have identified certain underground experiments as a top priority for their fields in their longrange plans. Efforts to develop a major facility in the United States have resulted in a proposal for a facility, the Deep Underground Science and Engineering Laboratory (DUSEL), to be located in the abandoned Homestake gold mine. ${ }^{4}$

The research to take place at DUSEL is described by the proponents as being built upon "four pillars," or four physics quests of critical scientific importancethe search for dark matter, the study of neutrino oscillations, and investigations into whether protons decay and whether atoms can undergo neutrinoless double-beta decay. In the proposed initial suite of experiments, these four quests are addressed by the apparatus of three experiments (see Chapter 3 ). The proponents of DUSEL also describe three research tenets-that the facility provide opportunities for a diverse set of research efforts in subsurface engineering, the geosciences, and biosciences; that it allow other well-motivated experiments to take advantage of the unique capabilities of a world-class underground research facility; and that it provide a significant education and outreach program for visitors and the communities near the laboratory.

The principal underground laboratory space is to be located at $4,850 \mathrm{ft}$, where plans call for installing five or six physics experiments and at least one earth science experiment. The proponents' plans also call for a deeper site, at 7,400 ft, where two smaller physics experiments and an earth science experiment would be located. Other research facilities could be installed at other levels, depending upon requirements of the experiments. Recently plans were developed that would allow for the installation of a liquid argon detector for the neutrino oscillation experiment at

\footnotetext{
${ }^{1}$ R. Davis, Jr. 1964. Solar Neutrinos: II. Experimental. Physical Review Letters 12: 303.

${ }^{2}$ R. Becker-Szendy, C.B. Bratton, D.R. Cady, et al. 1990. Search for proton decay into $e^{+}+\pi^{0}$ in the IMB-3 detector. Physical Review D 42: 2974-2976.

${ }^{3}$ D.S. Akerib, J. Alvaro-Dean, M.S. Armel-Funkhouser, et al. 2004. First results from the cryogenic dark matter search in the Soudan Underground Laboratory. Physical Review Letters 93: 211301.

4 "How much better to get wisdom than gold, to choose understanding rather than silver." Proverbs 16:16 (New International Version).
} 
$800 \mathrm{ft}$, where ramp, as opposed to vertical, access can be provided. Finally, the facility would include a large research space on the surface to support the underground experiments and allow for the development of future experiments.

A significant personnel and facilities infrastructure is called for to manage the ongoing facility as well as to plan for future expansion. Management must ensure that the facilities needs of each experiment—sufficient excavated space, ventilation, power, data transfer capabilities, and possible shielding, among others-are met. Safety concerns are paramount for space so deep underground, so measures would be taken to develop, maintain, and ensure compliance with all safety standards. Because one of the principal experiments at DUSEL would be the neutrino oscillation experiment associated with a neutrino source at the Fermi National Accelerator Laboratory (Fermilab), management would have to coordinate the scientific and facility development taking place at DUSEL with similar efforts at Fermilab.

The program would also include a process for evaluating the merit of proposed future experiments. Once selected, those experiments would be integrated with the current suite of experiments, either by incorporating them into the existing space or by excavating new space and expanding support services.

\section{This Study}

As part of the process for developing the DUSEL program, the National Science Foundation (NSF) and the Department of Energy (DOE) jointly commissioned this study. The principal charge to the committee is as follows:

The committee will undertake an assessment of the proposed DUSEL program, including:

- An assessment of the major physics questions that could be addressed with the proposed DUSEL and associated physics experiments,

- An assessment of the impact of the DUSEL infrastructure on research in fields other than physics,

- An assessment of the impact of the proposed program on the stewardship of the research communities involved,

- An assessment of the need to develop such a program in the U.S., in the context of similar science programs in other regions of the world,

- An assessment of broader impacts of such an activity, including but not limited to education and outreach to the public.

This report is the response to that charge. However, several events transpiring over the course of this study caused the committee to interpret the statement of task in a manner that merits explanation. Just before the committee's first meeting, in December 2010, the National Science Board (NSB), the governing organization for the NSF, elected not to provide bridge funding for the further development of the DUSEL facility. NSF's FY2012 budget request, submitted several months thereafter, 
indicated that the decision not to provide this funding was part of a larger determination by the NSB that the scope and likely cost of the project lay outside NSF's core mission. As a consequence, NSF would not be proceeding as principal steward of the DUSEL facility. The committee was also informed that there would be very limited follow-up to the preliminary design report being prepared by proponents of the DUSEL program and that it would mainly serve as general input for evaluating future opportunities. As it releases this report, the committee understands that DOE and NSF have been discussing whether to proceed with some or all of what has been described as the DUSEL program but that no firm decision has been made.

Given these uncertainties and developments, the committee has interpreted the portions of its charge by which it is to assess the science that might take place in the proposed DUSEL program (the first and second bullets in the statement of task) as directing the committee to evaluate the intellectual merit of the science to be addressed by the slate of experiments that were to be included in the initial DUSEL program, as described at the committee's first meeting. In particular, the committee did not assess any future experimental opportunities that would be enabled by the existence of an underground research facility but that had not been included in the initial suite of experiments. In assessing the impact of the DUSEL infrastructure on fields other than physics, the committee considered the suite of experiments in the biosciences, geosciences, and subsurface engineering that were presented to it as being indicative of the type of nonphysics questions that could be addressed rather than as specifying the DUSEL nonphysics program. The committee assessed the science questions in the general context of frontier research worldwide; it did not compare them with any particular alternative project or investment.

In responding to the remaining bulleted items in the statement of task-the impact such a program would have on the stewardship of research communities; the need to develop such a program in the United States given similar science programs elsewhere; and the broader impacts of such a program - the committee elected not to restrict its assessment to the specifics of "the proposed DUSEL program" at the Homestake site. Rather, it set forth more general considerations that it believes should be taken into account by policy makers in evaluating the impact that an underground laboratory facility in the United States-either a DUSEL-like national laboratory or a more limited facility-would have on advancing the goals of the U.S. research communities. In conformity with the charge, the committee assessed only the options associated with developing some form of underground research facility in the United States and did not assess the project costs or budgetary impacts of the facilities and experiments discussed.

This chapter provides an overview of the science questions that an underground research facility could address and of the broader impacts such a facility would have on the relevant research communities and summarizes the report's principal findings and conclusions. Chapter 2 discusses the general parameters of 
underground research space and the status of the principal underground research facilities around the world. Chapter 3 contains a detailed assessment of the principal science questions that would be addressed with the DUSEL program. Chapter 4 concludes the report by describing the broader impacts of the program, including the education and outreach opportunities such a program might provide.

\section{SCIENCE OVERVIEW}

\section{Physics Experiments}

The committee finds that three of the proposed physics experiments - a direct detection dark matter experiment on the scale of one to tens of tons; a long-baseline neutrino oscillation experiment; and a ton-scale, neutrinoless double-beta decay experiment — to be of paramount and comparable importance. These experiments are judged to be of paramount scientific importance because each would be a central component of an ongoing science program that would seek to address at least one crucial unanswered question whose eventual answer will greatly enhance our scientific understanding. As a consequence, each experiment would have the potential to make a breakthrough discovery upon which the future of particle, nuclear, and astrophysics will build. In this sense, each of these three experiments is essential and could radically transform scientific understanding and progress.

\section{Dark Matter}

The direct detection dark matter experiment will provide unprecedented sensitivity for the direct detection of the dark matter omnipresent in the Universe and an opportunity to discover the unknown particle nature of dark matter. Because dark matter makes up approximately 80 percent of the material Universe, the discovery of its nature would be of enormous significance to the fields of astrophysics and particle physics. The direct detection of dark matter would complement its indirect detection by astrophysics and accelerator-based searches for its production. Direct detection would strongly support the idea that dark matter candidates identified at an accelerator are indeed the mysterious dark matter pervading the Universe. Experiments to directly detect dark matter must be massive in order to capture weakly interacting dark matter particles. Experiments at the ton scale $(\sim 1$ ton to tens of tons, depending upon the technology) will achieve the sensitivity levels needed to discover and study dark matter. The background from cosmic rays that mimic the signals of dark matter demand that the experiments be performed underground. Moreover, the challenges to instrumentation posed by residual backgrounds demand that the world should have at least two experiments of this scale implementing different techniques. Because resolving the nature of dark matter is 
so vital and U.S. scientists have had a leading role in addressing this problem, the United States should take a leading role in mounting one of the two direct detection dark matter experiments on this scale, and support for U.S. scientists participating in the second direct detection experiment, wherever it is, would be appropriate.

\section{Neutrino Oscillation}

The long-baseline neutrino oscillation experiment would provide a great advance in the study of neutrino properties, particularly when coupled with a neutrino beam produced at Fermilab using a new high-intensity proton source that is under development. By significantly improving sensitivity to the so-called mixing angle between the lightest and heaviest of the neutrinos, to the hierarchy of neutrino masses, and to matter-antimatter asymmetry in neutrino oscillations, this experiment will probe for more signs of the new physics that revealed itself when neutrino mass was discovered in earlier oscillation experiments, and it will elucidate the processes of the early Universe. As discussed in more detail later in this chapter, this experiment will also provide increased sensitivity for the possible detection of proton decay and neutrinos from supernovas. Although these rare phenomena are not very likely to be observed, the detection of either proton decay or neutrinos from a nearby supernova would be of momentous scientific significance.

The detection and identification of neutrinos require massive, sensitive detectors to capture the weakly interacting neutrinos. Detectors that weigh between tens and hundreds of kilotons, depending on the technology, are required for sensitivity to the neutrino-antineutrino asymmetries, and underground sites are needed to control experimental backgrounds, with depth depending also on detector technology. Two detector technologies, the traditional water Cherenkov detector and a more novel liquid argon tracking calorimeter, are under study. Both would require a scaling up of present detectors to achieve the sensitivities needed to advance the fields. Accumulating enough information to untangle the subtle interplay of neutrino parameters demands intense neutrino beams as well as massive detectors. With its plans to exploit the full potential of the existing Fermilab accelerator complex to provide a high-intensity neutrino beam, and with the possibility of an even higher intensity neutrino beam generated by Project $X$ at Fermilab, the United States will be in a good position to conduct the next-generation long-baseline neutrino experiment.

\section{Neutrinoless Double-Beta Decay}

The neutrinoless double-beta decay experiment could determine whether neutrinos are their own antiparticles and could measure, or at least constrain, the neutrino masses. Resolution of the particle-antiparticle question will contribute in a critical way to our understanding of how particles came into existence in 
the early Universe and of why matter dominates over antimatter in the Universe today and will therefore contribute to our understanding of how the Universe has evolved. This experiment is the only practical way to address the particle-antiparticle question. Moreover, the masses of the neutrinos are fundamental parameters of the Standard Model and they cannot be accessed directly by studying neutrino oscillations.

Neutrinoless double-beta decay experiments must be massive in order to contain enough nuclei to allow observation of such rare decays. Experiments at the ton scale may be needed to achieve the sensitivity required to observe neutrinoless double-beta decay and/or to determine the neutrino masses. As with the direct detection of dark matter, backgrounds from cosmic rays that mimic the signals sought demand that the experiments be performed underground, and the challenges posed by residual backgrounds to the design of detector instrumentation demand that the world have at least two ton-scale experiments using different experimental techniques. Because it is so important to resolve the particle-antiparticle nature of the neutrinos and to determine their mass scale, and because U.S. scientists have been playing a leading role in addressing this challenge, the United States should take a leading role in mounting one of the ton-scale experiments, and the support of U.S. scientists participating in the other such experiments, perhaps elsewhere in the world, would be appropriate.

Together, these two neutrino experiments - the long-baseline neutrino experiment and the neutrinoless double-beta decay experiment-form a complementary program to address the outstanding questions of neutrino physics and to complete our understanding of this portion of the particle world and its key cosmological role.

The marked improvements in sensitivity that will be afforded by next-generation underground experiments for detecting dark matter and studying neutrinos will enable significant advances in these matters of fundamental and critical scientific importance. Proceeding with plans to build in the United States a worldleading long-baseline neutrino oscillation experiment, and taking a leadership role in developing within the United States both a direct detection dark matter experiment on a scale of one to tens of tons and a neutrinoless double-beta decay experiment on the ton scale will bring an extraordinary opportunity for the U.S. scientific community. The program would put U.S. scientists in a good position to have leadership in these crucial experimental undertakings. The benefits to the U.S. particle and nuclear physics communities would be greatest if, in addition to the neutrino oscillation experiment, the dark matter and neutrinoless double-beta decay experiments are both installed at a U.S. facility. However, if a U.S. site is not available for one or both of them, a U.S.-led experiment at an appropriate facility abroad would still be of significant benefit to the U.S. research communities. (See Chapter 4 for a discussion of appropriate facilities.) 
Conclusion: Three underground experiments to address fundamental questions regarding the nature of dark matter and neutrinos would be of paramount and comparable scientific importance:

- The direct detection dark matter experiment,

- The long-baseline neutrino oscillation experiment, and

- The neutrinoless double-beta decay experiment.

Each of the three experiments addresses at least one crucial question upon whose answer the tenets of our understanding of the Universe depend.

Conclusion: The three major physics experiments would not only provide an exceptional opportunity to address scientific questions of paramount importance, they would also have a significant positive impact upon the stewardship of the particle physics and nuclear physics research communities, and would have the United States assume a visible leadership role in the expanding field of underground science. The U.S. particle physics program is especially well positioned to build a world-leading long-baseline neutrino experiment due to the combined availability of an intense neutrino beam from Fermilab and a suitably long baseline from the neutrino source to an appropriate underground site such as the proposed DUSEL. In light of the leading roles played by U.S. scientists in the study of dark matter and doublebeta decay, together with the need to build two or more large experiments for each of these two areas, U.S. particle and nuclear physicists are also well positioned to assume leadership roles in the development of one direct detection dark matter experiment on the ton- to multiton scale and one neutrinoless double-beta decay experiment on the scale of a ton. While installation of such U.S.-developed experiments in an appropriate foreign facility or facilities would significantly benefit scientific progress and the research communities, there would be substantial advantages to the communities if these two experiments could be installed within the United States, possibly at the same site as the long-baseline neutrino experiment.

An underground research facility would also offer the opportunity to undertake several other important physics studies. In addition to investigating neutrino characteristics, the detector in the long-baseline neutrino oscillation experiment would provide the increased sensitivity needed for the study of proton decay and would allow collecting valuable data if a supernova event occurred in the nearby universe during the course of operation. The facility could also be the site for an accelerator-based study on nuclear cross sections that are critical for understanding a wide array of astrophysical events. 


\section{Proton Decay}

The massive detector of the long-baseline neutrino oscillation experiment will have a sensitivity to proton decay greater than that of current detectors. The stability of the proton is a matter of considerable scientific interest. There are compelling theoretical reasons to expect that the proton is unstable, and proton decay would provide a unique and direct window onto the physics of the GUT and the origin of matter. Because the lifetime of the proton is very long, the probability of observing any individual proton decay is very small, necessitating very massive detectors and an extremely large number of protons. As noted above, the detector of the long-baseline neutrino oscillation experiment will be more massive than previous detectors, allowing more sensitivity that could produce a major discovery. Nevertheless, the detectors being proposed are only large enough to improve sensitivity for many important decay modes by less than an order of magnitude over a 10-year operational period relative to what current instruments could achieve over the same time. As a result, the added reach is not sufficient for proton decay to be the primary factor in decisions on neutrino detector technology or siting.

\section{Supernova Studies}

The large detector of an underground long-baseline neutrino oscillation experiment could make a unique and valuable contribution to the study of supernovas. These remarkable phenomena play a crucial role in the history of the Universe as well as in the life of galaxies, yet they are not well understood. Detection of neutrinos from a supernova would provide a wealth of information about the dynamics of supernovas not available from astronomical observations, making this capability a significant feature of a long-baseline neutrino experiment. (Either a water Cherenkov detector or a liquid argon detector would detect a large number of neutrinos from a supernova in our galaxy.) Nonetheless, although a supernova (SN1987A) has previously been observed by particle detectors, supernovas close enough to Earth to be observed occur only rarely, approximately once or twice per century, and none may occur during the long lifetime of the experiment. However, should a nearby supernova occur, having more than one large neutrino detector in the world would ensure its observation and maximize the scientific output.

Conclusion: Two additional capabilities of the long-baseline neutrino experiment would be of great scientific interest and would add significant value to that experiment:

- Its sensitivity to the study of proton decay and

- Its sensitivity to the detection of neutrinos from supernovas. 
The stability of the proton is a crucial, fundamental scientific question. Moreover, the detection of neutrinos from supernovas would make a unique and valuable contribution to our understanding of one of the most important astrophysical phenomena. However, these sensitivities are not so important as to make them primary considerations in choosing neutrino detector technology or a site for the experiment.

\section{Nuclear Astrophysics}

The committee found that an accelerator-based study to measure the lowenergy nuclear cross sections needed to elucidate astrophysical processes would be scientifically important. These cross sections are critical for advancing our understanding of the nuclear processes that generate stellar energy and explain certain aspects of solar neutrinos and the abundances of the elements and their isotopes in the Universe. For example, with the recent greatly improved measurements of the solar neutrino flux, nuclear cross sections are now the dominant uncertainty in using the neutrino flux to extract information on neutrino properties as well as on solar structure and composition. Measuring nuclear cross sections at stellar energies requires high luminosities and low backgrounds, which can be provided by an underground accelerator facility. Such a facility would be an effective complement to the new Facility for Rare Isotope Beams (FRIB) in advancing nuclear astrophysics science. Because a large number of cross section measurements are needed and the cross sections to be measured have low counting rates, more than one such low-energy facility is called for worldwide. The proposed U.S. facility would thus enable scientifically important measurements beyond the number that can be made at the LUNA facility at the Gran Sasso Laboratory in Italy.

Conclusion: A small underground accelerator to enable measurements of low-energy nuclear cross sections would be scientifically important. These measurements are needed to elucidate fundamental astrophysical processes such as thermonuclear reactions and the production of heavy elements in the Sun and the stars.

\section{Nonphysics Experiments}

\section{Subsurface Engineering, the Geosciences, and the Biosciences}

Access to extensive underground space and the ability to conduct regulated longterm and large-scale tests would afford unparalleled research opportunities for fields 
such as subsurface engineering, the geosciences, and the biosciences. Such opportunities would inform the study of the complex hydraulic, chemical, mechanical, and thermal forces at play underground and how they interact in existing fracture systems subject to tectonic and gravitational forces, affecting deformation and slippage, producing earthquakes at faults, and influencing life in its extreme forms.

Conclusion: The ability to perform long-term experiments in the regulated environment of an underground research facility could enable a paradigm shift in research in subsurface engineering and would allow other valuable experiments in the geosciences and biosciences.

\section{BROADER IMPACTS}

\section{Co-location of Experiments}

Underground research such as the major physics experiments described above, requires experienced personnel and extensive infrastructure to provide access, power, and ventilation, as well as surface facilities for the assembly and maintenance of apparatus. Safety is, as always, important, particularly because of the inherent danger in working underground. Much infrastructure and personnel could be efficiently and effectively shared among contemporary experiments located at a single site, and among future experiments as well. A common site could also provide other benefits, such as opportunities for increased interactions and synergy among scientists engaged in different experiments. It could also heighten the visibility of the research, to the public here and to the international research community abroad.

Conclusion: The co-location of the three main underground physics experiments at a single site would be a means of efficiently sharing infrastructure and personnel and of fostering synergy among the scientific communities. The infrastructure at the site would also facilitate future underground research, either as extensions of the initial research program or as new research initiatives. These additional benefits, along with the increase in visibility for U.S. leadership in the growing field of underground science, would be important considerations when choosing a site for the three main physics experiments.

Conclusion: If co-located with one or more of the main underground physics experiments in the United States, a small underground accelerator facility to enable measurements of low-energy nuclear cross sections important to 
nuclear astrophysics would benefit from shared infrastructure, personnel, and expertise.

Conclusion: In light of the potential for valuable experiments in subsurface engineering, the geosciences, and the biosciences that could be offered by an underground research facility, if such facility is constructed in the United States for physics experiments, scientists in other fields would greatly benefit by having a mechanism in place that would allow them to perform research there.

\section{National Facility}

Access to underground research laboratories is vital to research programs in particle and nuclear physics and to the biological, geological, and subsurface engineering sciences of the subsurface. Indeed, underground facilities are essential for addressing some of the most important questions in science, such as the nature of the neutrino, the stability of the proton, and the nature of dark matter-and they enable valuable long-term experiments in a regulated environment. There are at present underground laboratories in several places in the world. However, the growing number of underground experiments and the need for multiple experiments for the direct detection of dark matter, for neutrinoless double-beta decay, and for nuclear astrophysics, and the growing size of those experiments mean increased demand for underground laboratory space around the world. For this reason, many of the world's existing laboratories have plans—as yet unrealized-for expansion, and there are proposals, most notably in China, to create an international underground facility. The Soudan Underground Laboratory, in Soudan, Minnesota, is the only general underground research laboratory in the United States, although some underground experiments are also performed at DOE's Waste Isolation Pilot Plant (WIPP) and the Sanford Underground Laboratory at Homestake, a facility developed in conjunction with the DUSEL program. Although each proposed or future experiment could be located in some existing, expanded, or new facility, stewardship of the research communities requires providing them access to adequate, appropriate underground research facilities. A national underground research facility in the United States would supplement and complement facilities elsewhere in the world by providing increased underground research space and future expansion capability, as well as appropriate infrastructure and safety systems. Although the final decision to build a national underground facility will be made taking into account many other factors, including the programmatic goals of the funding agencies and the costs of different options, significant advantages 
would accrue to the pertinent U.S. research communities if such a facility were to be built here. ${ }^{5}$

Conclusion: A facility for underground research would have a significant positive impact on the stewardship of the research communities involved. Such a facility would offer the particle and nuclear physics communities access to the underground research space they need to undertake a range of scientifically critical experiments, and it would allow the bioscience, geoscience, and subsurface engineering communities to perform valuable longterm experiments in a regulated environment.

An underground research facility in the United States could offer advantages over underground facilities in other places in the world. Foremost, a large neutrino oscillation experiment in this country could be coupled with the present and future capabilities of the Fermilab accelerator complex, which would provide an intense neutrino beam at a suitably long baseline, making the United States a world leader in neutrino physics. At present, no other location in the world offers a fully competitive combination of future neutrino intensity and an appropriate underground site for a very large neutrino detector. Furthermore, some underground science programs, such as the programs that are imperative in direct detection of dark matter and neutrinoless double-beta decay, require multiple large experiments using complementary techniques. As these international programs evolve, it becomes reasonable to expect that the hosting and supporting of large experiments will be shared by underground laboratories in different countries. Meanwhile, biological and, particularly, geological and subsurface engineering experiments need to be performed in many different environments. The site proposed for the DUSEL program offers some special features for particular engineering science experiments. Finally, an underground research facility in the United States would offer advantages to the U.S. research communities, reinforcing their stewardship of the research. It would provide a research site that does not involve travel to distant places and would facilitate graduate student training as well as the research

\footnotetext{
${ }^{5}$ Following the NSB's decision not to proceed with stewardship of the DUSEL program, the DOE initiated a study to evaluate the financial aspects of several options, including funding a facility with many of the components of the original DUSEL program and funding a program in which several experiments are constructed at the Sudbury Neutrino Observatory near Sudbury, Ontario, Canada. February 28, 2011, Letter from the Office of the Director, Department of Energy, to Dr. Jay Marx and Mr. Mark Reichanadter. The results of that study were made available around the time of the release of this report. Available at http://www.dusel.org/dusel/recent/Marx_Review_of_Underground_Science_ Report_Final.pdf. Last accessed on October 19, 2011.
} 
enterprise. It could also help ensure access to underground space for experiments led by U.S. scientists, who have historically been leaders in underground science. At the same time, it would guarantee the United States a leadership role in the expanding global underground science community while being a principal component of the growing U.S. world-class particle physics program at the Intensity Frontier.

Conclusion: Development of an underground research facility in the United States would supplement and complement underground laboratories around the world. A U.S. facility could build upon the unique position of the United States that would allow it to develop a long-baseline neutrino experiment using intense beams from Fermilab. It could accommodate one of the large direct detection dark matter experiments and one of the large neutrinoless double-beta decay experiments that are needed by the international effort to delve into these critical scientific issues, while sharing infrastructure among the three experiments, which are of comparable import. It could also host and share infrastructure with other underground physics experiments, such as an accelerator to study nuclear astrophysics, and with underground experiments in other fields. An underground research facility would benefit the U.S. research communities and would guarantee the United States a leadership role in the expanding global field of underground science. 


\section{2 \\ Description of Underground Facilities}

\section{GENERAL CHARACTERISTICS OF UNDERGROUND LABORATORIES}

The appropriateness of an underground facility for a particular experiment depends on a number of its characteristics. Typically the most important of these is the effective depth of the laboratory and therefore the degree to which backgrounds associated with cosmic rays are reduced. In addition to the vertical distance from the surface, often referred to as the facility's "overburden" or "vertical overburden," the structure, density, and makeup of the earth above the laboratory impact the penetration capability of cosmic rays. A facility's depth is therefore "normalized" by measuring the actual cosmic ray intensity at that facility and then expressing its depth in terms of meters of water equivalent (m.w.e.), or the equivalent depth of water that would reduce the cosmic ray intensity to the measured amount. Figure 2.1 shows the measured drop-off in cosmic ray muon intensity as a function of m.w.e. Typically, the depth of a facility expressed in m.w.e. is roughly 2.65 times its vertical overburden expressed in meters.

An underground facility's appropriateness for a given experiment also might be impacted by the absence or availability of active shielding that can be shared by several experiments. This typically is a "shield" outside the inner main detector that is often itself an active detector. By measuring activity at the shield and providing that information to the experiment, the influence of the surrounding environment on the inner main detector can be estimated and accounted for in data analysis. 


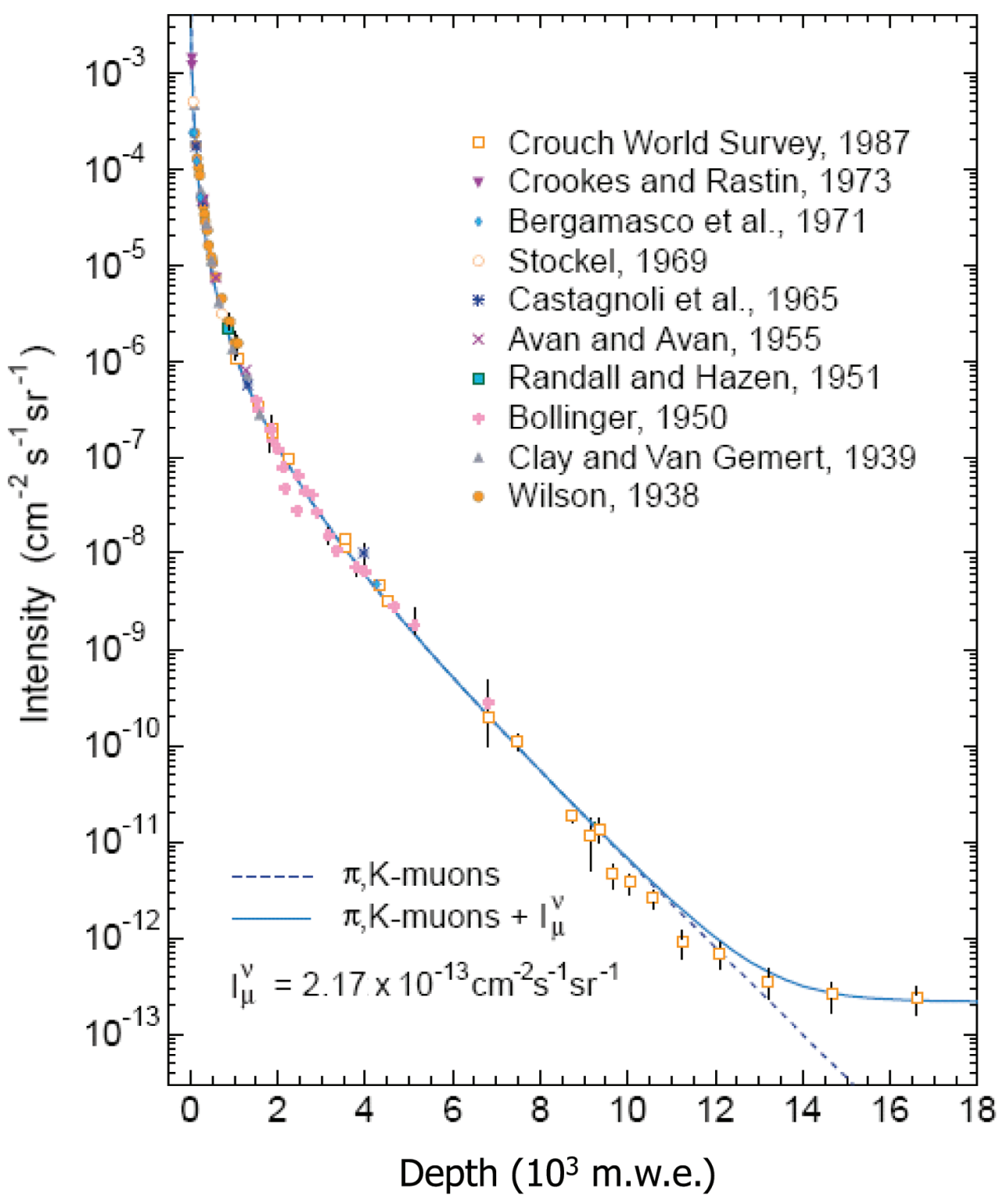

FIGURE 2.1 Cosmic ray muon intensity as a function of depth, expressed as meters of water equivalent. SOURCE: Reprinted with permission from E.V. Bugaev, A. Misaki, V.A. Naumov, T.S. Sinegovskaya, S.I. Sinegovsky, and N. Takahashi. 1998. Atmospheric muon flux at sea level, underground, and underwater. Physical Review D 58: 054001. Copyright 1998 by the American Physical Society. 
Still other important characteristics include how the laboratory space is accessed, whether that access is shared, and the nature of the rock surrounding the laboratory. Horizontal shafts that allow the use of vehicles to bring equipment and supplies into and out of the laboratory space typically are preferred to vertical shafts, especially where those shafts, and the lifts in them, are fairly small. Many laboratories coexist with working mines or vehicular tunnels, so access to the laboratory space can at times be limited. The type of rock from which the laboratory was excavated can be of importance, especially for larger experiments. As the size of experiments grows, the density and stability of the surrounding rock and its ability to support the weight of the experimental apparatus could become an issue.

Finally, the comprehensiveness and location of the support facilities for an underground laboratory can be important general characteristics. Most of these facilities are located on the surface and typically include shared items such as electricity, communications, and cold water for the cooling of the experimental apparatus. Further, each underground laboratory needs a support team to care for safety, technical support, transportation between the surface and underground, and the like, which can vary significantly from facility to facility.

\section{SURVEY OF SELECTED LABORATORIES}

Underground research facilities are scattered throughout the world. Figure 2.2 shows the size and effective depth, in m.w.e., of the principal underground laboratories, including the program proposed for DUSEL. The remainder of the chapter discusses the characteristics of the principal laboratories located or planned in North America-DUSEL, Soudan, and SNOLAB — as well as Gran Sasso, the largest underground laboratory in the world; Kamikande, the largest Asian laboratory; and, briefly, CDUSEL/China JinPing Laboratory (CJPL), a laboratory being developed in China that is expected to be large, on the scale of DUSEL. Appendix $\mathrm{D}$ contains more detailed information about all of the principal laboratories shown in Figure 2.2. In developing this material, the committee drew from the results of two recent comprehensive surveys of underground laboratories. ${ }^{1}$

\footnotetext{
${ }^{1}$ A. Bettini. 2011. Underground laboratories. Nuclear Instruments and Methods in Physics Research Section A: Accelerators, Spectrometers, Detectors and Associated Equipment 626: S64-S68; E. Coccia. 2010. Underground laboratories: Cosmic silence, loud science. Journal of Physics Conference Series 203: 012023.
} 


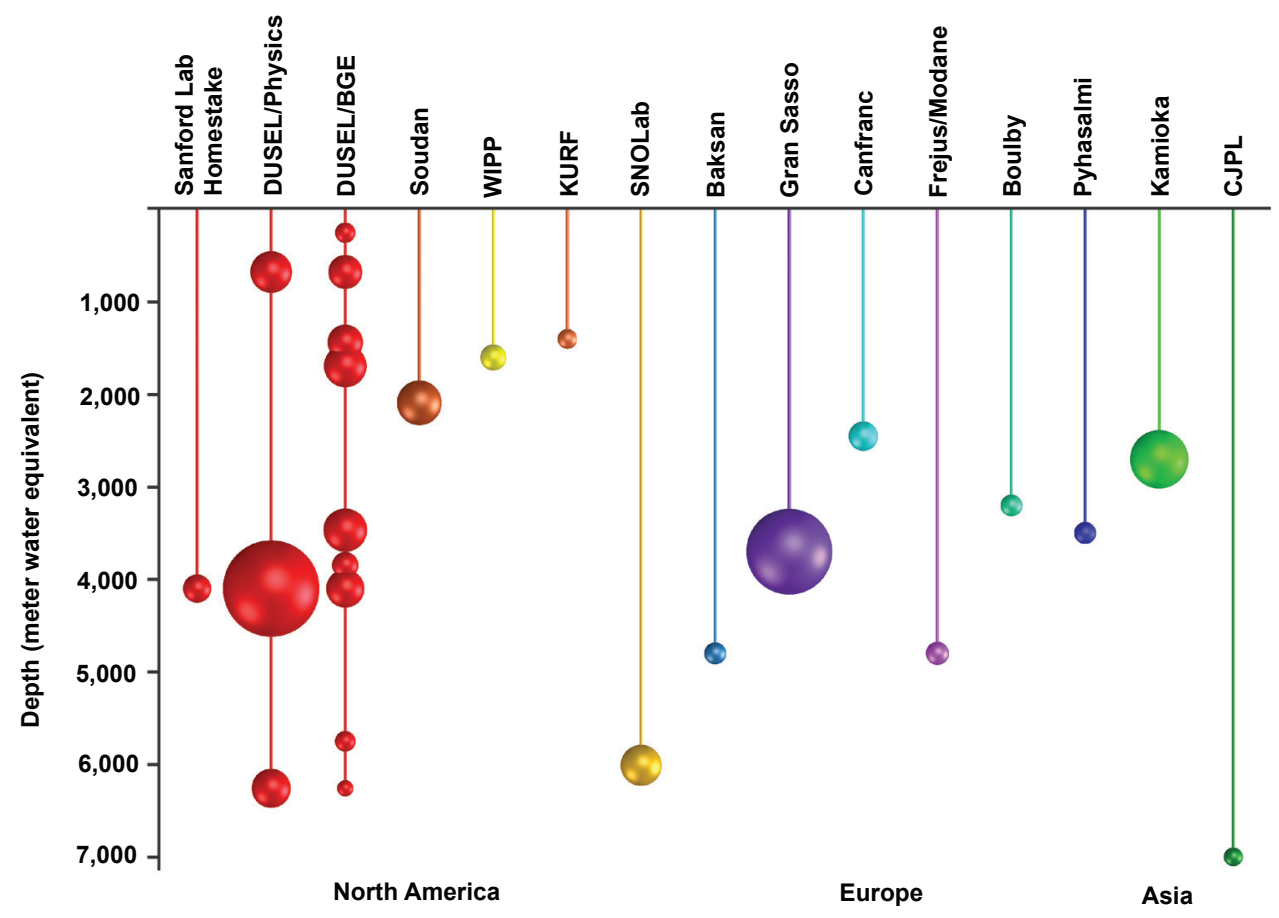

FIGURE 2.2 Depths and relative volumes, represented by the size of the spheres, of the principal underground laboratories in the world. The red spheres are associated with the Homestake mine, where the DUSEL program was designed to be placed. The only underground laboratory at the Homestake mine in existence at the time this report was written is the left-most sphere associated with the Sanford Underground Laboratory. The depths shown are for vertical direction and generally have the effect of exaggerating the shielding provided by mountain sites such as Gran Sasso and Kamioka relative to flat sites such as Soudan. SOURCE: Image courtesy of the University of California at Berkeley and the DUSEL Project; K. Lesko, University of California at Berkeley, "Deep Underground Science and Engineering Laboratory (DUSEL) Project Overview," Presentation to the committee on December 14, 2010.

\section{Deep Underground Science and Engineering Laboratory/ Sanford Underground Laboratory (United States)}

The proposed DUSEL program was developed by a group of researchers based at the University of California at Berkeley pursuant to an award granted by NSF. The program calls for a multilevel facility at the Homestake mine, an abandoned gold mine in Lead, South Dakota. In addition to surface facilities, the principal underground facilities would be located at the 800-, 4,850-, and the 7,400-ft levels, with the opportunity to place additional small experiments at various other levels, depending upon the demands of the research. 
The surface campus, as planned, consisted of approximately $27,000 \mathrm{~m}^{2}$ of research and administrative space, with $1,100 \mathrm{~m}^{2}$ of that for assembly of experiments. Administrative and science support, including shops, offices, and assembly sites, were to be located at this level. It was also envisioned to have a separate maintenance and operations campus and a facility for education and outreach. The size of the facility at $800 \mathrm{ft}$ depended, to a large degree, on whether there would be a liquid argon detector for the neutrino oscillation/proton decay experiments, as discussed in Chapter 3, in the section entitled "Neutrino Physics."

At $4,850 \mathrm{ft}$, it was proposed to have $25,000 \mathrm{~m}^{2}$ of total working space, with $6,200 \mathrm{~m}^{2}$ devoted to science. This level would be the principal deep underground laboratory space and the site for the water Cherenkov detector(s), if chosen for the neutrino oscillation and proton decay experiments, as well as the dark matter and nuclear astrophysics experiments. The deepest level, at 7,400 feet, would consist of 7,000 $\mathrm{m}^{2}$ total space, with $1,300 \mathrm{~m}^{2}$ dedicated to research. It would be available for possible dark matter and neutrinoless double-beta decay experiments, as well as ecohydrology, geoscience, and subsurface engineering experiments.

There are currently several small experiments under way at the 4,850 foot level as part of the Sanford Underground Laboratory. This laboratory was established in anticipation of the full-scale implementation of the DUSEL program, but its future is uncertain in light of changes to the DUSEL program. For more information, see http://dusel.org.

\section{Soudan Underground Laboratory (United States)}

Soudan Underground Laboratory (SUL) is the only general research underground laboratory currently located in the United States. This facility was developed in 1980 and installed in an abandoned iron mine in Minnesota. The underground structures include the $1,400 \mathrm{~m}^{2}$ principal laboratory space, which hosts a dark matter experiment and a low-background counting facility; a small, high-purity copper fabrication facility; and the $560 \mathrm{~m}^{2}$ main injector neutrino oscillation search (MINOS) laboratory. The MINOS experiment is the far detector in a neutrino oscillations experiment; its neutrino beam originates at Fermilab. MINOS is expected to run a few years more with a 2-year decommissioning period at the end of that time.

The laboratory is fairly shallow, with a vertical overburden of $700 \mathrm{~m}$ of rock, and access is through a small, vertical shaft whose size places some restrictions on installation capability. The laboratory offers some education and outreach to the general public. It coexists with a historic state park that offers mine tours, some of which utilize a visitor's gallery in the MINOS laboratory. For more information, see http://www.soudan.umn.edu/. 


\section{Sudbury Neutrino Observation Laboratory (Canada)}

The Sudbury Neutrino Observatory (SNO) was excavated in the 1990s in an operating nickel mine. The original SNO experiment, located in a $200-\mathrm{m}^{2}$ area, had a very successful set of discoveries and has completed its run. To this original space, new structures have been added to form a new laboratory, the SNOLAB, which consists of a main hall with $270 \mathrm{~m}^{2}$ floor area and ceiling heights from 15 to $19.5 \mathrm{~m}$, a service hall of about $180 \mathrm{~m}^{2}$, and a number of narrow volumes called "ladder labs." The laboratory space is one of the deepest currently available, with a rock coverage of 2,000 m under a flat surface. A "cryopit," designed to cope with the safety issues surrounding large volumes of cryogenic fluids, has also been excavated. The total underground laboratory area is $7,215 \mathrm{~m}^{2}$, of which $3,055 \mathrm{~m}^{2}$ is available for experiments.

The access is through a vertical shaft that is shared with the working mine and is available daily. All of the laboratory space will be maintained at Class 1500 cleanliness standards. On the surface a 3,159- $\mathrm{m}^{2}$ building hosts a clean room, laboratories, staging and assembly areas, and administrative space.

The scientific programme includes (1) the Project in Canada to Search for Supersymmetric Objects (PICASSO), which is searching for dark matter (2 kg) using the superheated bubbles technique; (2) the experiment $\mathrm{SNO}+$, which is to be hosted in the former SNO cavity and will use a liquid scintillator in which ${ }^{150} \mathrm{Nd}$ has been dissolved to study low-energy solar neutrinos, geoneutrinos, and doublebeta decay; and (3) dark matter searches that include the Dark matter Experiment with Argon and Pulse shape discrimination/Cryogenic Low Energy Astrophysics with Noble gases (DEAP/CLEAN), currently operating with a prototype, and superCDMS, operating with bolometers. For more information, see http://www. snolab.ca/ or http://www.sno.phy.queensu.ca/.

\section{Laboratori Nazionali del Gran Sasso (Italy)}

Laboratori Nazionali del Gran Sasso (LNGS) is a national laboratory of Italy's Istituto Nazionale di Fisica Nucleare (INFN). It is the largest underground laboratory in the world and serves the largest and most international scientific community, about 750 scientists from 26 countries. LNGS arose out of a proposal in 1979 that a large underground laboratory be built close to and in conjunction with the Gran Sasso freeway tunnel then under construction in central Italy (an opportunity that substantially reduced its cost). The Parliament approved the construction in 1982, and construction was completed in 1987.

Access is horizontal, via the freeway. The underground laboratory principally consists of three main halls, each with an area of about 2,000 $\mathrm{m}^{2}(100 \mathrm{~m} \times 20$ $\mathrm{m}), 18 \mathrm{~m}$ high. There are also ancillary tunnels that provide space for services 
and small-scale experiments and two 90-m long tunnels built for two Michelson interferometers for geology studies. The total area is $17,300 \mathrm{~m}^{2}$, and the total volume $180,000 \mathrm{~m}^{3}$. The laboratory is reasonably deep, with a vertical overburden of $1,400 \mathrm{~m}$. Services hosted on the surface campus include a full range of support and administrative facilities, and the laboratory organizes a number of outreach and education activities.

LNGS is operated as an international laboratory with an international scientific committee, appointed by INFN, which advises the director on the suite of experiments for the facility. The scientific program includes these:

- The search for $\tau$ neutrino appearance on the $\mu$ neutrino beam emitted from the Large Hadron Collider of the European Center for Nuclear Research (CERN), $732 \mathrm{~km}$ away. This is the main focus of the OPERA experiment, which uses emulsion techniques and a large (kiloton), sensitive mass, consisting of 150,000 bricks made up of lead sheets interleaved with emulsion layers.

- ICARUS, a general-purpose particle detector in a 600-ton liquid argon time-projection chamber.

- Solar neutrino physics and geoneutrinos with the 1,300-ton liquid scintillator Borexino detector.

- The detection of low-energy neutrinos from the gravitational collapse of galactic objects with the 1,000-ton liquid scintillator Large Volume Detector (LVD) experiment.

- Dark matter searches with LIBRA (250-kg sensitive mass of NaI crystals), CRESST2 (an ultracryogenic $\mathrm{CaWO}_{4}$ detector), XENON (liquid xenon) and WARP (liquid argon)

- Neutrinoless double-beta decay experiments with GERDA (enriched $\left.{ }^{76} \mathrm{Ge}\right)$, CUORE $\left(\mathrm{TeO}_{2}\right.$ bolometers), and COBRA (CdZnTe semiconductor detectors).

- Nuclear reaction of astrophysical interest with a 400-kV accelerator with LUNA2.

A special facility is dedicated to low radioactivity measurements, and the laboratory also supports several experiments in geology, biology, and the environment. For more information, see http://www.lngs.infn.it/.

\section{Kamioka Observatory (Japan)}

The Kamioka Observatory is operated by the Institute for Cosmic Ray Research, University of Tokyo. It was established in 1983 by M. Koshiba as the Kamioka Underground Observatory. The original purpose of this observatory was 
to conduct the Kamioka neutron decay experiment (KamiokaNDE); later, a neutrino observatory, Super-Kamiokande (SuperK), was built, which is currently the largest underground experiment. The Tokai-to-Kamioka long-baseline neutrino oscillation experiment $(\mathrm{T} 2 \mathrm{~K})$ recently began operations. It is a third-generation neutrino oscillation experiment on an intense off-axis beam of muon neutrinos $\left(v_{\mu}\right)$ produced at the J-PARC accelerator facility $295 \mathrm{~km}$ from the SuperK detector. The main goal of T2K is to measure the oscillation of $v_{\mu}$ to $v_{e}$ and to measure the value of $\theta_{13}$.

The average vertical overburden for the research space is $1,000 \mathrm{~m}$, and access is horizontal by vehicle, with no interference from mining activity. The average number of scientific users is more than 200 .

The underground structures comprise the following:

- Hall SK (50-m diameter) hosting Super-Kamiokande;

- Clean room $\left(10 \times 5 \mathrm{~m}^{2}\right)$ with XMASS prototype;

- Hall 40 (L-shape, $40 \mathrm{~m} \times 4 \mathrm{~m}$ arms) hosting the purification tower for XMASS and the NEWAGE experiment on dark matter;

- Hall 100 (L-shape, $100 \mathrm{~m} \times 4 \mathrm{~m}$ arms) with CLIO, a prototype of gravitational antenna (to be terminated in 2013) and a laser displacement detector;

- The new Hall A $\left(15 \times 21 \mathrm{~m}^{2}\right)$ hosting XMASS $800 \mathrm{~kg}$; and

- The new Hall B $\left(6 \times 11 \mathrm{~m}^{2}\right)$ hosting CANDLE on double-beta decay, to be occupied until 2012.

Small areas are available in the abandoned mine. The underground large cryogenic gravitational antenna (LCGT), which has baseline lengths of $3 \mathrm{~km} \times 3 \mathrm{~km}$, was recently approved. Further enlargements are under development in order to accommodate more experiments. Buildings for offices and computer facilities are available on the surface.

In the same mountain, the Kamioka Liquid Scintillator Antineutrino Detector (KamLAND) experiment is operated by the Neutrino Centre, Tohoku University. KamLAND is designed to detect electron antineutrinos and to provide important results for neutrino oscillation by measuring antineutrinos from the commercial power reactors surrounding the site. For more information, see http://www-sk. icrr.u-tokyo.ac.jp/index_e.html

\section{China Deep Underground Science and Engineering Laboratory, aka China JinPing Deep Underground Laboratory (China)}

Recently, the China Deep Underground Science and Engineering Laboratory (CDUSEL) (also known as CJPL), a project for the world's deepest, and possibly 
largest, underground laboratory, was launched in China. ${ }^{2}$ The facility plans to take advantage of infrastructure being developed by the Ertan Hydropower Development Company (EHDC) in the course of installing a series of 21 hydroelectric power stations on the Yalong River in central China. A system of tunnels $17.5 \mathrm{~km}$ long will cut a big U-turn in the river under the 4,193-m-tall JinPing mountain. This system will have a flat area available for development as an underground laboratory that provides at its greatest depths a 2,500 m vertical rock overburden and more than $1,500 \mathrm{~m}$ vertical overburden in 70 percent of the directions. The access will be horizontal, from both sides.

Two small experimental halls $5 \times 5 \times 30 \mathrm{~m}^{3}$ are under construction; their relative size is shown in Figure 2.2. The final size of the laboratory has not been publicly disclosed, although it has been reported that the laboratory will be designed as an international facility, open to the world community. Ventilation, laboratory-grade power supply, and germanium detectors with their shielding will be installed. The muon flux (expected to be very low, on the order of 20 per $\mathrm{m}^{2}$ per year), the neutron flux, and radon concentration in the air will be measured shortly. A working group including scientists and engineers from Chinese institutions and universities as well as EHDC has been established to develop plans for this facility.

\footnotetext{
${ }^{2}$ Qian, Yue. 2010. Status and prospects of China JinPing deep underground laboratory (CJPL) and China dark matter experiment (CDEX). Presentation at the TeV Particle Astrophysics 2010 Conference, Paris, France.
} 


\section{3 \\ Science Assessments}

The proposed DUSEL science program encapsulates an initial suite of physics experiments and diverse multidisciplinary research experiments in subsurface engineering, the geosciences, and the biosciences and has the capacity for more future experiments. This chapter undertakes to present the committee's assessment of the main physics questions to be addressed by the proposed physics experiments and of the impact of the proposed facility on research in fields other than physics. The proposed physics experiments are one or more dark matter experiments; a longbaseline experiment for the study of neutrino oscillations and proton decay that is also capable of measurements in neutrino astrophysics; a neutrinoless doublebeta decay experiment; and an accelerator-based nuclear astrophysics experiment. Accordingly, the chapter assesses, in no particular order, the physics questions of dark matter, of long-baseline neutrino oscillations and neutrinoless double-betadecay in the larger context of neutrino physics and, together with proton decay, in the context of unified theories; of nuclear astrophysics, and of neutrino astrophysics. It also undertakes an assessment of the impact of the proposed laboratory infrastructure on research in fields other than physics-namely, subsurface engineering and the geosciences and biosciences.

To give an idea of the scale of the experiments needed to address the elements of the proposed DUSEL program, the construction cost ranges estimated by the DUSEL project during the preliminary design process were $\$ 80$ million to $\$ 200$ million for the dark matter experiment(s); $\$ 785$ million to $\$ 1,065$ million for the long-baseline neutrino and proton decay experiment, $\$ 250$ million to $\$ 350$ million for the neutrinoless double-beta decay experiment, $\$ 30$ million to $\$ 50$ million for 
the nuclear astrophysics facility, and $\$ 60$ million to $\$ 180$ million total for multiple experiments in subsurface engineering and geoscience and bioscience. The estimated incremental costs associated with efforts to detect supernovas and proton decay are not significant. Budgetary considerations and further development of the experiments will, of course, change the actual costs of these experiments.

Because both the DUSEL program and the designs for the experiments to address the critical physics questions are still evolving, the committee chose to focus its assessment on the scientific merits of the questions to be addressed rather than on the technical merits of the experiments as they are now designed. Accordingly, it did not assess the technical merits of each experiment being sited at DUSEL or the suitability of alternative sites. Similarly, the committee chose to focus its assessment on the general scientific merits of research in the fields other than physics that would be enabled by the availability of an underground research facility rather than on the specific scientific or technical merits of a particular suite of nonphysics underground experiments. In choosing to focus in this way, the committee intends its assessments to be of value to the future direction of underground research, independent of whether the DUSEL program, as presently conceived, is realized. Finally, the committee assessed the intellectual merit of the underground science of the proposed DUSEL program in the general context of frontier scientific research worldwide. It was not a purpose of this study to rank the different fields or subfields of science, or to prioritize across programs. Neither the individual science questions nor the overall scientific program were compared with those of any other particular projects or investments.

\section{PHYSICS PROGRAM}

\section{Dark Matter}

\section{Overview}

Astronomers are sure that what can be detected by telescopes represents only a small portion of the Universe; furthermore, only a small fraction ( $\sim 4$ percent) is made of normal matter of the type that we live with here on Earth and observe directly elsewhere. The remainder of the Universe is composed of dark matter (about 22 percent), which has mass but does not emit or absorb light, and dark energy (about 74 percent). While dark energy is best studied using astrophysical techniques, direct detection of dark matter in the laboratory is possible, and direct experimental detection of dark matter interactions would profoundly change our understanding of both the microscopic world of elementary particles and the macroscopic astrophysical world, thus bridging the very smallest and the very largest objects in the known Universe. 
The first evidence for the existence of dark matter came from observations of the rate at which astronomical objects such as stars, gas clouds, and galaxies rotate. It was discovered that bodies far from the center of rotation move faster than would be predicted using the laws of gravity and the visible mass of known objects, suggesting that unseen bodies existed on a grand scale. Additional evidence for dark matter comes from cosmological observations such as the fluctuation patterns of the cosmic microwave background, and further corroboration is provided by observations of colliding galaxies where the dark matter has been imaged using gravitational lensing. Depictions of this phenomenon have captured the imagination of the general public (see Figure 3.1).

Many explanations of the composition of dark matter have been proposed and compared with experimental data. Some of the dark matter could come from unobserved dark bodies of ordinary matter, such as massive compact halo objects or molecular gas clouds. However, to understand cosmological data requires the existence of exotic dark matter, and there now is consensus that most of the dark matter consists of as-yet-undiscovered elementary particles whose nature has yet to be determined. One possibility motivated by theory is that the dark matter arises from a particle called the axion. Experimental searches for axions and indirect astrophysical detection of dark matter use techniques that do not operate underground and so will not be discussed here. A second theoretically attractive possibility is that dark matter consists of weakly interacting massive particles (WIMPs). Such WIMPs could be directly detected in underground experiments and would be the focus of an underground dark matter search program.

\section{Scientific Landscape}

Theories of elementary particle physics provide natural candidates for WIMPs. For example, in many supersymmetric models, the lightest supersymmetric particle is stable, and many of these theories naturally provide particles with masses and interaction cross sections that are consistent with astronomical and cosmological bounds on WIMP properties. There are also nonsupersymmetric theories that postulate the existence of particles with the appropriate properties. Several of these particles are being searched for in accelerator-based programs such as the Large Hadron Collider (LHC) of the European Organization of Nuclear Research (CERN). However, only the direct detection of naturally occurring WIMPs would assure that these particles, whether discovered at an accelerator or not, are in fact the source of dark matter.

Because they are elementary particles not found in the Standard Model, it is likely that, when discovered, dark matter particles will be a central ingredient in finding solutions to known problems with present particle theory. Knowledge of the mass, the interaction rate, and the number density of dark matter particles 


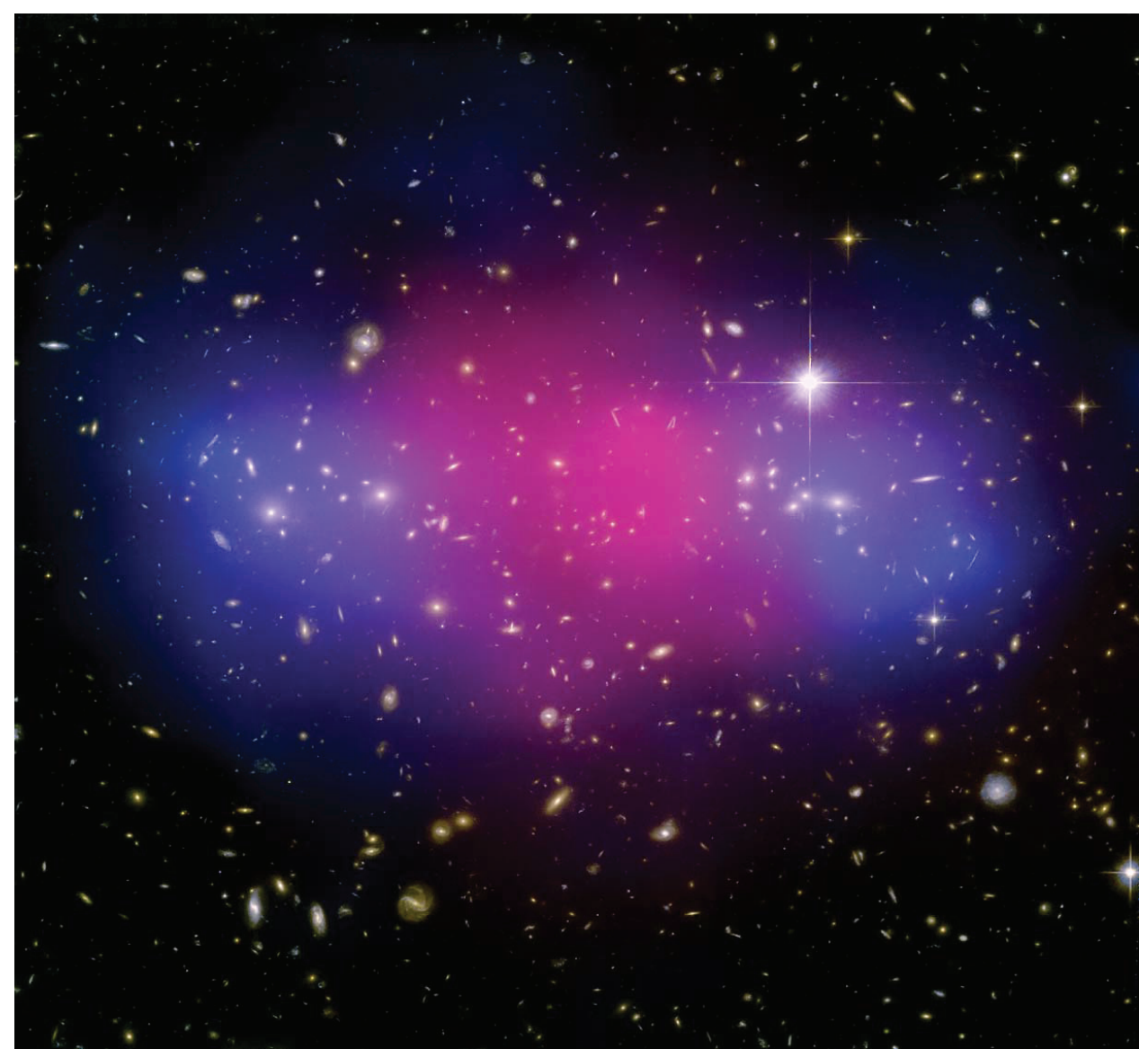

FIGURE 3.1 Color-coded image of colliding galaxies, with familiar matter shown in red (from x-rays) and dark matter shown in blue (modeled from weak lensing measurements). The interactions of familiar matter slow the collision, while the weakly interacting dark matter associated with each galaxy is essentially transparent and so passes through. The cluster, known as MACS J0025.4-1222, is a composite of separate exposures from the Hubble Telescope and the Chandra observatory. Astronomers say the images may shed light on the behavior of dark matter. SOURCE: $x$-ray image, National Aeronautics and Space Administration/Chandra X-ray Center/Stanford University/S. Allen; optical lensing image, National Aeronautics and Space Administration/Space Telescope Science Institute/ University of California at Santa Barbara/M. Bradac. 
independent of any theoretical framework would allow predictions of production and annihilation rates that could be tested in future experiments. These data would also affect cosmological calculations relevant for describing the evolution of the Universe.

\section{Experimental Aspects}

The direct detection of dark matter would involve the search for collisions between ordinary nuclei and WIMPs from the halo of our galaxy. Such observations would be difficult, since WIMPs interact rarely and the signals of the collision would be very faint. Therefore, detectors having a good likelihood of measuring such collisions would need to be large and operate deep underground to reduce backgrounds of cosmic ray origin that can mimic the signals being sought.

These searches are based on the hypothesis that dark matter consists of WIMPs with a mass of a few tens of proton masses or greater. When such a particle collides with a target it should produce a recoiling nucleus whose energy can be measured through scintillation light flashes, phonons, or ionization produced by the nucleus. Learning to address the challenges associated with these types of studies requires a series of experiments with ever-increasing target mass and improvements in methods for rejecting background signals. History teaches that each generation of detector corresponds to an increase of about an order of magnitude in target mass. In the 25 years since WIMPs were first proposed as a dark matter candidate, the sensitivity of nuclear recoil experiments has improved by a factor of more than 1 billion. Once irreducible backgrounds are encountered for a specific detector, further running in the same configuration improves sensitivity only very slowly. It is much more efficient to determine appropriate solutions to identify and account for backgrounds and then to incorporate these improvements while also increasing the target mass.

Past experiments are referred to as generation zero (G0) and ongoing experiments as generation one (G1). G1 experiments typically operate with tens of kilograms of target mass and are reaching much better background reduction and sensitivity than G0 experiments. Experience with the targets and the handling of backgrounds have informed next-generation designs, and G2 experiments are currently under development and installation. These experiments will have hundreds of kilograms of target mass, and the following generation, G3, will have even greater target masses, 0.5 to multiple tons. The experiments considered for DUSEL are in the G3 category.

The dark matter experiments summarized in Table 3.1 illustrate the current and future generations of detector and techniques. U.S. scientists historically have been heavily involved in this research and are expected to continue their involvement. The compelling nature of the science, and the high discovery potential, 
makes it important that they do so and that opportunities exist for discoveries to be made in the United States.

The strategy for experimental background rejection depends on which of the three signatures currently used to observe the nuclear recoil is chosen: scintillation, phonons, or ionization. Some experiments use a "single signature," including the shape and localization of that signal. These include single-phase noble liquid (xenon, argon) scintillation experiments and experiments exploiting the bubble chamber concept, where ionization in a supersaturated liquid creates bubbles that can be detected visually or acoustically or both.

Other experiments, including most of the leading large experiments, use combinations of two signatures to reinforce background rejection: (1) light/ionization together with phonons or heat in crystals at millikelvin temperatures and (2) light/ionization in noble liquid detectors. Experiments of the first kind use germanium or scintillating crystals; those of the second are double-phase ionization/ scintillation xenon or argon experiments, so called because they operate under conditions where the gas and liquid phases coexist, enabling amplification of the weak ionization signal in the gas. Research and development are under way on direction-sensitive detectors using low-pressure-gas "time projection chambers." Debates regularly surface in the dark matter community about whether certain experiments have properly excluded or included claims of positive signals. To address these uncertainties about signals, it is important that a single experiment be able to collect multiple complementary signals and that multiple experiments using different nuclear targets are conducted.

The most recent results over the WIMP mass range of 10-1,000 GeV exclude cross sections approaching $10^{-44} \mathrm{~cm}^{2}$ per nucleon for the simplest models (see Figure 3.2). However, the DAMA/Libra experiment has a long-standing observation with an annual modulation of the event rate that is consistent with a WIMP having a mass of less than $10 \mathrm{GeV}$. These results have persisted over 7 years of data taking. However, the cross section indicated by the DAMA/Libra experiment is not consistent with limits from the CDMS and XENON-100 experiments in most WIMP models. There are also measurements that may indicate an excess above backgrounds at very low WIMP masses, but this signal is not as well established as the DAMA/Libra observation. Finally, a number of cosmic ray experiments (PAMELA and ATIC) report excess electron or positron signals that could be from WIMP annihilation in our galaxy. It is, however, somewhat complicated to find dark matter models that reconcile these results with the charged cosmic ray data from the Fermi/LAT experiment. However, several research groups have pointed out that conventional astrophysical sources of positrons could account for the putative PAMELA/ATIC signal. The theoretical community has been very active in trying to explain some or all of these results and has developed new models leading to new signals to search for at the LHC, in B-factory data and in electron-scattering 
TABLE 3.1 Plans of WIMP Search Collaborations Using Nondirectional Detectors Around the World

\begin{tabular}{|c|c|c|c|c|c|c|}
\hline \multirow{2}{*}{$\begin{array}{l}\text { Country/ } \\
\text { Region }\end{array}$} & \multicolumn{2}{|c|}{ Current Generation (G1) } & \multicolumn{2}{|c|}{ Generation 2 (G2) } & \multicolumn{2}{|c|}{ Generation 3 (G3) } \\
\hline & Gross Mass & Current Status & Gross Mass & Current Status & Gross Mass & Current Status \\
\hline United States & $\begin{array}{l}\text { LUX } \\
350 \mathrm{~kg} \mathrm{Xe} \\
\text { Sanford Lab }\end{array}$ & $\begin{array}{l}\text { Assembly } \\
2011 \text { Install }\end{array}$ & $\begin{array}{l}\text { LZS } \\
1.5-3 \text { tons Xe } \\
\text { Sanford Lab }\end{array}$ & $\begin{array}{l}\text { Design } \\
\text { Same water } \\
\text { tank as LUX }\end{array}$ & $\begin{array}{l}\text { LZD } \\
20 \text { tons Xe } \\
\text { DUSEL }\end{array}$ & $\begin{array}{l}\text { S4, R\&D } \\
2017\end{array}$ \\
\hline $\begin{array}{l}\text { U.K./ } \\
\text { Portugal/ } \\
\text { Russia }\end{array}$ & $\begin{array}{l}\text { ZEPLIN III } \\
10 \mathrm{~kg} \mathrm{Xe} \\
\text { Boulby, U.K. }\end{array}$ & $\begin{array}{l}\text { Running } \\
(2009-2010)\end{array}$ & & & & \\
\hline United States & $\begin{array}{l}\text { Darkside-50 } \\
50 \mathrm{~kg} \mathrm{Ar} \\
\text { LNGS }\end{array}$ & $\begin{array}{l}\text { Design } \\
\text { DAr under } \\
\text { procurement } \\
2011-2012\end{array}$ & 1 ton & $\begin{array}{l}\text { Design } \\
\text { Same } \\
\text { shield as } \\
\text { DarkSide-50 }\end{array}$ & $\begin{array}{l}\text { MAX } \\
6 \text { tons Xe } \\
20 \text { tons DAr } \\
\text { DUSEL }\end{array}$ & $\begin{array}{l}\text { S4, R\&D } \\
2017 \text { Install }\end{array}$ \\
\hline $\begin{array}{l}\text { United States/ } \\
\text { Europe/ } \\
\text { China }\end{array}$ & $\begin{array}{l}\text { XENON100 } \\
80 \mathrm{~kg} \\
\text { Gran Sasso }\end{array}$ & Running & $\begin{array}{l}\text { XENON1T } \\
2.4 \text { tons Xe }\end{array}$ & $\begin{array}{l}\text { Design } \\
2012 \text { Install }\end{array}$ & & \\
\hline $\begin{array}{l}\text { United States/ } \\
\text { Canada }\end{array}$ & $\begin{array}{l}\text { SCDMS } \\
10 \mathrm{~kg} \mathrm{Ge} \\
\text { Soudan }\end{array}$ & $\begin{array}{l}\text { Construction } \\
2011 \text { Install }\end{array}$ & $\begin{array}{l}\text { SCDMS } \\
100 \mathrm{~kg} \mathrm{Ge} \\
\text { SNOLAB }\end{array}$ & $\begin{array}{l}\text { R\&D } \\
2014 \text { Install }\end{array}$ & $\begin{array}{l}\text { GEODM } \\
1.5 \text { tons } \\
\text { DUSEL }\end{array}$ & $\begin{array}{l}\text { S4, R\&D } \\
2018\end{array}$ \\
\hline United States & $\begin{array}{l}\text { COUPP } \\
60 \mathrm{~kg} \mathrm{CF}_{3} \\
\text { SNOLAB }\end{array}$ & $\begin{array}{l}\text { Construction } \\
\text { NUMI test } \\
2010\end{array}$ & $500 \mathrm{~kg}$ & $\begin{array}{l}2011 \text { Design } \\
2013 \text { Install }\end{array}$ & 16 ton scale & $\begin{array}{l}\text { S4 } \\
R \& D\end{array}$ \\
\hline Canada & $\begin{array}{l}\text { PICASSO } \\
2.6 \mathrm{~kg} \\
\text { SNOLAB }\end{array}$ & Running & $\begin{array}{l}\text { PICASSO II } \\
25 \mathrm{~kg}\end{array}$ & $\begin{array}{l}\text { 2010/11 } \\
\text { Install }\end{array}$ & $\begin{array}{l}\text { PICASSO III } \\
>500 \mathrm{~kg}\end{array}$ & 2012/13 Install \\
\hline $\begin{array}{l}\text { United States/ } \\
\text { Canada }\end{array}$ & $\begin{array}{l}\text { MiniCLEAN } \\
500 \mathrm{~kg} \mathrm{Ar}\end{array}$ & $\begin{array}{l}\text { Construction } \\
2011 \text { Install }\end{array}$ & $\begin{array}{l}\text { DEAP- } 3600 \\
3.6 \text { tons }\end{array}$ & $\begin{array}{l}\text { Funded } \\
2012 \text { Install }\end{array}$ & $\begin{array}{l}\text { CLEAN } \\
50 \text { tons Ar/Ne }\end{array}$ & $\begin{array}{l}\text { Planning } \\
\text { R\&D }\end{array}$ \\
\hline Europe & $\begin{array}{l}\text { Edelweiss } \\
\text { Now } 3 \mathrm{~kg} \rightarrow \\
24 \mathrm{~kg} \mathrm{Ge} \\
2011 \text { Modane }\end{array}$ & $\begin{array}{l}\text { Running } \\
24 \mathrm{~kg} \\
\text { funding } \\
\text { secured }\end{array}$ & $\begin{array}{l}\text { EURECA } \\
100 \mathrm{~kg} \mathrm{Ge} \\
\text { interleaved } \\
\text { Ge/scintillator, }\end{array}$ & $\begin{array}{l}\text { Active R\&D } \\
2013 \text { Install }\end{array}$ & $\begin{array}{l}\text { EURECA } \\
1 \text { ton Ge/ } \\
\text { Scintillator, } \\
\text { LS Modane }\end{array}$ & $\begin{array}{l}\text { Planning } \\
2016\end{array}$ \\
\hline Europe & $\begin{array}{l}\text { CRESST } \\
5 \mathrm{~kg} \text { of CaWO } \\
\text { Gran Sasso }\end{array}$ & Running & $\begin{array}{l}\text { Modane } \\
\text { extension, } \\
\text { Merging of } \\
\text { CRESST and } \\
\text { Edelweiss }\end{array}$ & & extension & \\
\hline Europe & $\begin{array}{l}\text { ArDM } \\
800 \mathrm{~kg} \mathrm{Ar} \\
\text { Canfranc }\end{array}$ & $\begin{array}{l}\text { Construction } \\
2011 \text { Install }\end{array}$ & & & & \\
\hline $\begin{array}{l}\text { Europe/ } \\
\text { United States }\end{array}$ & $\begin{array}{l}\text { WARP } \\
140 \mathrm{~kg} \text { Ar } \\
\text { Gran Sasso }\end{array}$ & Running & & & & \\
\hline
\end{tabular}


TABLE 3.1 Continued

\begin{tabular}{|c|c|c|c|c|c|c|}
\hline \multirow{2}{*}{$\begin{array}{l}\text { Country/ } \\
\text { Region }\end{array}$} & \multicolumn{2}{|c|}{ Current Generation (G1) } & \multicolumn{2}{|c|}{ Generation 2 (G2) } & \multicolumn{2}{|c|}{ Generation 3 (G3) } \\
\hline & Gross Mass & Current Status & Gross Mass & Current Status & Gross Mass & Current Status \\
\hline \multirow[t]{3}{*}{ Japan } & XMASS & Installation & XMASS II & $R \& D$ & XMASS III & Planning \\
\hline & $800 \mathrm{~kg} \mathrm{Xe}$ & Running & 5 tons & 2014 Install & 10 tons & 2016 \\
\hline & Kamioka & 2010 & & & & \\
\hline China & $\begin{array}{l}\text { JinPing lab } \\
\text { Ge and/or Xe }\end{array}$ & Planning & $100 \mathrm{~kg}$ & 2015 R\&D & $>1$ ton & 2020 \\
\hline
\end{tabular}

NOTE: All masses are the active masses of the central detectors. DAr, Ar depleted in ${ }^{39} \mathrm{Ar}$; LUX, Large Underground Xenon experiment; LZS, 1,500 to 3,000 kg liquid xenon detector; LZD, 20-ton liquid xenon detector; S4, NSF Solicitation 4; ZEPLIN III, two-phase Xe detector; Darkside, Depleted Argon [K]ryogenic Scintillation and Ionization Detector; MAX, Multiton Argon and Xenon detector; LNGS, Laboratori Nazionali del Gran Sasso; XENON100, Xenon 100-kg dark matter experiment; XENON1T, Xenon 1 ton dark matter experiment; SCDMS, Soudan Cryogenic Dark Matter Search; GEODM, Germanium Observatory for Dark Matter; COUPP, Chicagoland Observatory for Underground Particle Physics; MiniCLEAN, Mini-Cryogenic Low Energy Astrophysics with Noble liquids experiment; DEAP-3600, Dark matter Experiment using Argon Pulse-shape discrimination; CLEAN, Cryogenic Low Energy Astrophysics with Noble liquids experiment; EURECA, European Underground Rare Event Calorimeter Array; CRESST, Cryogenic Rare Event Search with Superconducting Thermometers; ArDM, Argon Dark Matter experiment; WARP, Wimp Argon Program experiment; XMASS, Xenon Dark Matter Search Experiment. SOURCE: Adapted from B. Sadoulet, University of California at Berkeley, "Dark Matter at DUSEL," Presentation to the committee on December 14, 2010.

experiments. At some level, dark matter imposes itself on every branch of particle physics. To keep all these communities from confusion as claims of discovery are made, definitive conclusions must be reached, and this will necessitate more than one detector that uses more than one technique.

In the next 4 to 6 years, with the deployment of the G2 experiments such as MiniCLEAN, DEAP-3600, and LUX, and as new results from XENON-100 become available, sensitivities can be expected to increase by another order of magnitude.

Two of the approaches under consideration for G3 detectors are 1-ton phononmediated low-temperature detectors and 1-ton or multiton noble liquids. U.S. scientists are playing leadership roles using both techniques, and contacts between this country and European groups are well developed. G3 experiments will push the cross section sensitivity below $10^{-47} \mathrm{~cm}^{2}$ per nucleon. Sensitivity near $10^{-48} \mathrm{~cm}^{2}$ per nucleon approaches a new background regime at which solar neutrino coherent scattering becomes important. This solar neutrino background is irreducible, and to progress past the regime, statistical background subtraction or directional detection become necessary, both of which represent a quantum step in difficulty. Thus, supporting G3 experiments are a natural goal for the next decade. On a longer timescale, large directional detectors may be required. Underground access for detector development is essential because background signals at the surface make it impossible to accurately assess performance aboveground. Post-G3 and large directional detectors would likely require large caverns at great depth. 


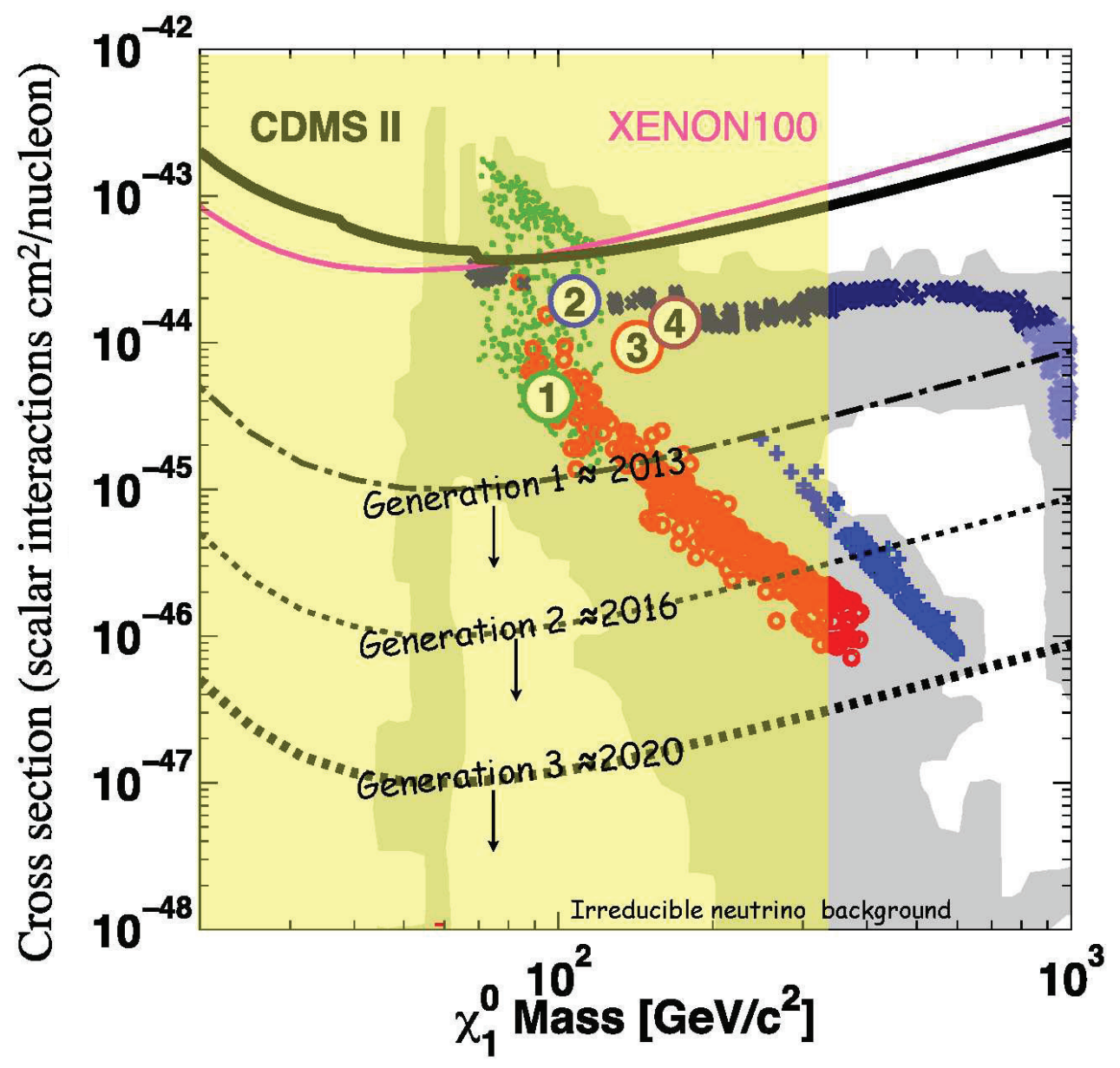

FIGURE 3.2 WIMP current limits (solid top curves) and sensitivity and goals (numbered) for the next 3 years; Generation 1 (G1), with results in 2013; Generation 2 (G2), with results in about 2016; and Generation 3 (G3), with results in about 2020. The shaded regions represent the expectation of several minimum supersymmetry models. SOURCE: Courtesy of Bernard Sadoulet, University of California at Berkeley, and Richard Gaitskell, Brown University.

Once a definitive dark matter signal is established, the next goal would be to observe the annual signal modulation as Earth's velocity relative to the dark matter halo changes owing to Earth's motion around the Sun. Such velocity effects, largest at the threshold energy of the detector, would take several years of operation to convincingly establish. An annual modulation signal would be compelling evidence and within the scope of a G2 or G3 experiment.

Beyond annual modulation, a detector with directional sensitivity could potentially observe a daily modulation of the direction of dark matter at all energies due 
to the finite rotational velocity at the surface of the Earth, thereby opening the door to dark matter astronomy. Directional detection would give information about the velocity distribution of WIMPs and would begin to discriminate between models of the dark matter halo. Directional detectors would rely on detecting the nuclear recoil in low-pressure gas and so represent a new technology. They would require large caverns and are not expected to be deployed before 2024 .

\section{Summary}

The predominant mass in the Universe is dark matter. Demonstrating that dark matter consists of elementary particles would be a major discovery. Understanding the nature and composition of these particles is a major scientific challenge for our time. ${ }^{1}$

The direct detection of dark matter would provide a crucial experimental connection between particle physics and cosmology. To be definitive, their signature signals would need to be significantly above the background and would need to come from different experiments. Concurrence between experiments will be essential: Several experiments have already claimed dark matter signals, but these have not been confirmed by other experiments. The program in dark matter detection will by necessity involve a number of G2 experiments that will coalesce into a smaller number of highly sophisticated and massive G3 detectors. Based on the history of leadership by U.S. physicists on experiments using all detection modes, it is expected that there will be U.S. involvement in more than one G3 experiment, and given the importance of this science and the discovery potential, it would be desirable for the United States to be a leader in at least one. Once dark matter has been observed, a major program for understanding the properties of the new particles will be required.

Conclusion: The direct detection dark matter underground experiment is of paramount scientific importance and will address a crucial question upon whose answer the tenets of our understanding of the Universe depend. This experiment would not only provide an exceptional opportunity to address a scientific question of paramount importance, it would also have a significant positive impact upon the stewardship of the particle physics and nuclear physics research communities and would have the United States assume a visible leadership role in the expanding field of underground science. In light of the leading roles played by U.S. scientists in the study of dark matter, together with the need to build two or more large experiments for this area,

\footnotetext{
${ }^{1}$ NRC. 2006. Revealing the Hidden Nature of Space and Time. Washington, D.C.: The National Academies Press, p. 13.
} 
U.S. particle and nuclear physicists are well positioned to assume leadership roles in the development of one direct detection dark matter experiment on the ton- to multiton scale. While installation of such a U.S.-developed experiment in an appropriate foreign facility would significantly benefit scientific progress and the research communities, there would be substantial advantages to the communities if this experiment could be installed within the United States, possibly at the same site as the long-baseline neutrino experiment.

\section{Tests of Grand Unification Theories}

The three other major physics experiments proposed for DUSEL-neutrino oscillations, neutrinoless double-beta decay, and proton decay-are among the most promising tests of theories that seek to provide a unified description of the forces. ${ }^{2}$ After providing a general overview of the nature of grand unification theories, these three experiments, and the roles they might play in resolving outstanding questions, are described.

We are able to observe the Universe because it contains important ingredients that are the stuff of ordinary matter: protons and neutrons, which are composites of quarks, and electrons. Whatever the history of the Universe, these particles were left behind and are stable enough to account for what is visible to us. Most of the properties and interactions of the visible matter made up of these particles can be accounted for by current particle theories. However, significant inconsistencies within existing theories and gaps in our knowledge remain. The remaining major physics experiments proposed for DUSEL should help fill those gaps and address those inconsistencies.

What is now called the Standard Model evolved throughout the twentieth century and aimed to describe the physics of these elementary particles and how they interact. In the Standard Model there are two fundamental fermion-type particles, as shown on the left side of Figure 3.3. They divide into six flavors of quarks and six types of leptons, three with a charge- the electron, the muon, and the tau - and their associated neutrinos. The quarks are strongly interacting fundamental particles that combine to make up the baryons (protons and neutrons); the leptons do not strongly interact. Each particle type is associated with a conserved quantum number. Quarks carry the baryon number, and baryon number conservation guarantees the stability of the proton and many nuclei. However, quarks also come in different flavors, and weak nuclear interactions can change one flavor of quark into another. The leptons carry lepton number L, and until the 1990s and

\footnotetext{
${ }^{2}$ The dark matter experiment discussed in the preceding section also has implications for tests of grand unified theories by way of the information it might provide on supersymmetry.
} 


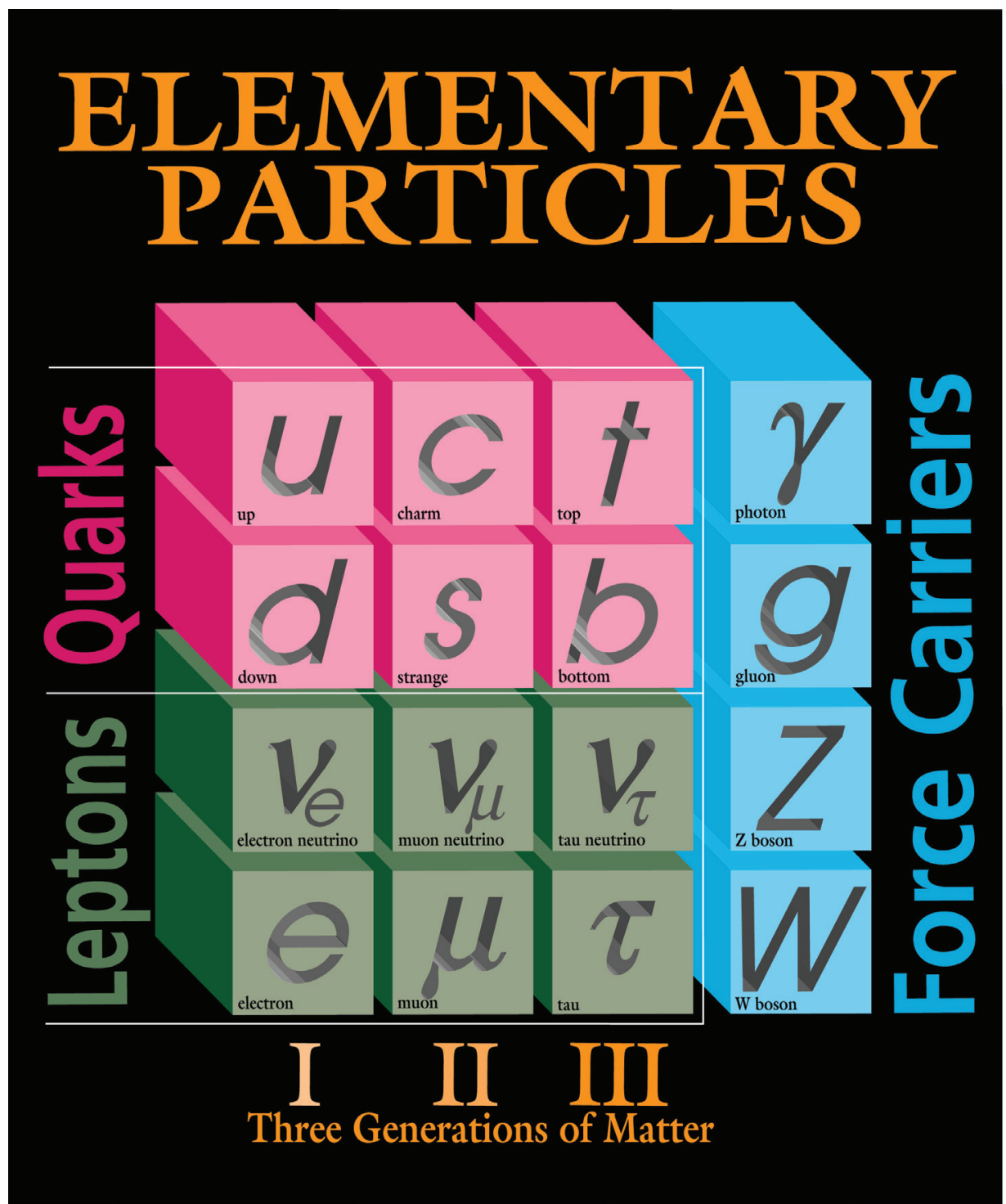

FIGURE 3.3 A complete list of the known fundamental particles as of April 2011. The twelve particles on the left are the fermions (the building blocks of matter), while those on the right (in blue) are the bosons (the force carriers). The charged leptons (electron, muon, and tau) are in the bottom row and their associated neutrinos in the row above. The quarks (red) engage in strong, or nuclear, forces while the leptons (green) do not. All these particles engage in weak nuclear forces. The neutrinos are the only fermions without electric charge and so are the only matter particles that do not engage in electromagnetic interactions. Matter in the "visible" Universe consists of quarks (inside protons, neutrons, and other nuclei) and electrons, either alone or bound in atoms. Much of this matter, along with the neutrinos, is stable. The list does not contain the unknown stable particles comprising the "invisible" dark matter. SOURCE: Courtesy of Fermilab. 
the discovery of neutrino oscillations, experimental observations were consistent with there being three separate conservation laws associated with the three lepton flavors-electron, muon, and tau lepton numbers.

In addition to the lepton and quark particles, there are four known fundamental forces-strong, electromagnetic, weak, and gravity. In any theory consistent with relativity and quantum mechanics, each force is associated with a boson-type particle, shown in the fourth column of Figure 3.3, which is the carrier of the force. In the Standard Model, the weak nuclear and electromagnetic forces are unified into the so-called electroweak interactions. The electromagnetic and weak interactions appear to be very different at low energy only because of the mass differences between their respective force carriers: The carrier of the electromagnetic force (the photon) is massless, while the carriers of the weak force (weak bosons) are approximately 100 times heavier than the proton.

A central question in particle physics is whether there are further unifications of forces. In the 1970s, the first grand unified theories (GUTs) unified the strong and electroweak interactions. From the broad class of GUTs emerges a unified picture of the quarks (strongly interacting fundamental particles) and the leptons that shares many similarities with the Standard Model. However, the GUTs differ from the Standard Model in several significant ways - for example, they predict that the proton is unstable and that there are bosons whose interactions can change a quark into a lepton or into an antiquark. Furthermore, unlike the Standard Model, in which neutrinos are massless, most GUTs predict that neutrinos would have tiny masses and would be their own antiparticles (Majorana-type particles) and that neither lepton number nor lepton flavor would be conserved. In GUTs, baryon and lepton number violations are associated with the exchange of new, extremely heavy force carriers, with masses $\sim 10^{15}$ times that of the proton, so that a process like proton decay exists but would be extremely rare. While direct production of such heavy force carriers will not be possible in any conceivable high-energy collider, proton decay may well be observable.

Cosmology also presents strong arguments in favor of many of the conclusions drawn from GUTs - that the proton is unstable, that neutrinos have mass, and that lepton flavor violation should be different for neutrinos and antineutrinos. The present day excess of matter over antimatter translates to an excess of quarks over antiquarks in the early Universe of about one part in $10^{8}$. In principle this excess could be an initial condition of the Universe; however, in standard cosmological explanations, inflationary expansion in the extremely early Universe would probably have removed any such initial excess. However, the Soviet nuclear physicist Andrei Sakharov pointed out that fundamental physics could produce a tiny excess of matter over antimatter during the early Universe provided three conditions are met: baryon number is not conserved, charge conjugation and 
charge parity (CP) are not symmetries of nature (nature distinguishes between matter and antimatter), and the early Universe went through a period when it was out of thermal equilibrium. If the first two conditions are met, the proton should decay.

GUTs offer a beautiful explanation for the origin of the asymmetry between matter and antimatter, which is deeply connected with CP violation in neutrino oscillations, tiny neutrino masses, and neutrino flavor change. In GUTs, the neutrino masses are inversely proportional to the masses of very heavy particles, so the tiny size of the neutrino masses suggests the existence of particles that are too heavy to be produced today but that could have been produced in the early Universe. Such particles decay out of equilibrium into leptons, violating CP and lepton number conservation, thus producing an excess of leptons over antileptons (a process known as "leptogenesis"). Anomalous electroweak processes then convert some of the excess leptons into quarks ("baryogenesis"). Thus CP violation in neutrino physics and the Majorana nature of neutrinos could well be linked to the origin of the matter excess in the Universe.

Some of the inconsistencies between the Standard Model and GUTs have been resolved. For example, it is now known (and described in the following section) that, contrary to the tenets of the Standard Model but consistent with GUTs, neutrinos have small masses and that lepton numbers are not conserved, as neutrinos oscillate between types. However, many other inconsistencies have not been resolved, and gaps remain in our knowledge of the characteristics of these most fundamental of particles. The studies proposed for DUSEL are highly promising experimental approaches for testing many of these outstanding questions-do neutrinos oscillate, are neutrinos their own antiparticles, and do protons decay? These proposed studies are discussed in the following sections.

\section{Neutrino Physics Overview}

Although neutrinos were first observed experimentally more than 50 years ago, their properties are less well understood than those of other elementary particles, in part because they have no electric charge and interact very weakly, making them difficult to detect. Like other matter particles, neutrinos have spin, a form of angular motion, but very small masses, weighing many million times less than any other matter particles. Neutrinos come in three different generations, called flavors, and each neutrino shares a flavor quantum number with a charged lepton partner: the electron, the muon, and the tau (shown below the neutrinos in the bottom row of Figure 3.3). While the weakness of neutrino interactions makes them difficult to observe, it also makes them ideal probes of certain astrophysical processes (see the section "Neutrino Astrophysics"), and the study of tiny masses 
may point to physics at far higher energies than could ever be reached with a terrestrial particle accelerator.

An interesting property of neutrinos is that a neutrino born with one flavor will spontaneously transform into another flavor as it moves through space. This phenomenon, known as "neutrino oscillation," has only been known for about 15 years. A similar phenomenon among quarks has been studied extensively, most recently at the two B-factories, one in the United States and the other in Japan. Scientific interest in such oscillations arises because they provide a mechanism whereby particles and their antiparticles can interact differently. Such difference between particle and antiparticle behavior is known as "CP violation" and is a key component of theories attempting to explain why the Universe is made primarily of matter, with very little antimatter. The amount of CP violation among quarks is insufficient to allow these theories to explain our matter-dominated Universe. However, CP violation involving neutrinos is an attractive theoretical way to explain this matter/antimatter imbalance through a process called leptogenesis, which was discussed in the preceding section.

Another interesting property of neutrinos is their relationships with antineutrinos. For each particle species, there is a corresponding antiparticle species. When particles have a charge, their antiparticle partners have the opposite charge, making these particles and antiparticles distinct. For particles like neutrinos, which have no net charge, it will have to be determined experimentally whether the particle and the antiparticle are different. In the Standard Model of particle physics, neutrinos have a lepton number and antineutrinos have a lepton number of the opposite sign. Since lepton number is conserved in the Standard Model, neutrinos and antineutrinos are distinguishable and their differences can be studied through their interactions with matter. In many theories that extend the Standard Model, however, lepton number is not conserved. In such theories, it is possible for neutrinos to be their own antiparticles, a property that would make them Majorana particles. The most promising experimental sign of neutrinos being Majorana particles would be the observation of a rare nuclear decay called neutrinoless double-beta decay. This fundamentally important process has been unsuccessfully sought for many decades but is expected to become observable in the next decade.

We are entering an era when the accurate determination of the properties of neutrinos needed for a deeper understanding of particle physics will be possible. There are still anomalies in the data and huge gaps in our knowledge, making this a very exciting time to gather largely unexplored information about this perplexing group of particles. The experiments considered here address the most critical open questions in neutrino physics. Because the answers that emerge will have major impacts on cosmology as well as on particle physics, this work represents an important scientific opportunity for the U.S. physics community. 


\section{The Nature of Neutrinos-Oscillations (Long-Baseline Neutrino Experiment)}

Electron neutrinos are the main product of the thermonuclear reactions that power the Sun. It has been about 40 years since Ray Davis and his collaborators first discovered evidence that their flux at Earth is substantially less than predicted by the best solar models of the time. ${ }^{3}$ S.M. Bilenky and Bruno Pontecorvo suggested that electron neutrinos change flavor in transit and become muon or tau neutrinos. ${ }^{4}$ Such neutrino oscillations require that neutrinos have mass. The ideas of both massive and flavor-changing neutrinos were revolutionary, and because the experimental evidence was not strong, the Standard Model of particle physics was constructed with massless and flavor-conserving neutrinos. Only within the last 15 years has the experimental evidence for neutrino oscillations become convincing enough for the scientific community to accept that they are a fact of nature.

To illustrate the most important phenomenological features of mixing, the case when only two neutrinos are involved will be considered. In a two-flavor world, the probability, $\mathrm{P}$, that one flavor $\left(\right.$ say, $v_{\mathrm{e}}$ ) will appear in a pure beam that was initially of another flavor $\left(\right.$ say, $\left.v_{\mu}\right)$ is given as

$$
P=\sin ^{2}(2 \theta) \sin ^{2}\left(1.27 \Delta \mathrm{m}^{2} \mathrm{~L} / \mathrm{E}\right)
$$

where $\theta$ is the mixing angle, $\Delta \mathrm{m}^{2}$ is the difference between the squares of the masses of the two neutrinos (in $\mathrm{eV}^{2}$ ), $\mathrm{L}$ is the distance (in kilometers) from the source to the detector, and $\mathrm{E}$ is the energy of the neutrinos (in $\mathrm{GeV}$ ). Of course the original flavor disappears at this same rate, so the total number of neutrinos remains constant.

This formula suggests two types of experiments: disappearance experiments, where a changing flux of the original flavor is observed as a function of distance or energy; and appearance experiments, where neutrinos of a different flavor appear in the beam. Note that the experimentally controllable parameters, the distance from the source and energy of the neutrinos, appear only in the ratio L/E. It is also noteworthy that oscillation experiments cannot determine the absolute masses of neutrinos since the probability $\mathrm{P}$ depends only on the difference between the squared masses. This formula for $\mathrm{P}$ also indicates how to measure the oscillation parameters - the amplitudes of the measured oscillations determine the mixing

\footnotetext{
${ }^{3}$ See, for example, R. Davis, D.S. Harmer, and K.C. Hoffman. 1968. Search for neutrinos from sun. Physical Review Letters 20: 1205.

${ }^{4}$ S.M. Bilenky and B. Pontecorvo. 1977. Lepton mixing and neutrino oscillations. Physics Review 41: 225.
} 
angles, while the variations with either distance or energy determine the mass squared differences.

An important feature was added when Stanislav Mikheyev and Alexei Smirnov, building on the earlier work of Lincoln Wolfenstein, realized that interactions of the neutrinos with electrons in the Sun (or even in Earth) could lead to a substantial modification of the oscillation probabilities, resonantly making amplitudes of oscillation either larger or smaller than otherwise. These matter, or MikheyevSmirnov-Wolfenstein ("MSW"), effects can be important in understanding data and are very useful in that they allow the neutrino masses to be ordered.

With the three known flavors of neutrinos, the oscillation phenomena are more complicated but also much richer in possibilities. The formulas governing the three flavor mixing are well understood and contain a number of independent parameters that govern neutrino flavor change and propagation:

- $\Delta \mathrm{m}_{21}^{2}$, the mass-squared difference primarily associated with solar neutrino disappearance. ${ }^{5}$ It has been measured thus far to be $\left|\Delta \mathrm{m}_{21}^{2}\right|=7.59 \pm 0.20$ ) $\times 10^{-5} \mathrm{eV}^{2}$.

- $\Delta \mathrm{m}_{32}^{2}$, the larger mass-squared difference primarily associated with atmospheric muon neutrino disappearance. It has been measured thus far to be $\left.\mid \Delta \mathrm{m}_{32}^{2} \mathrm{I}=2.35_{-0.08}^{+0.11}\right) \times 10^{-3} \mathrm{eV}^{2}$.

- $\theta_{12}$, the parameter known best for governing the disappearance of solar electron neutrinos; sometimes known as the solar mixing angle. It has been measured thus far to be large: $\sin ^{2} 2 \theta_{12}=0.87 \pm 0.03$.

- $\theta_{23}$, the parameter primarily known for its role in the disappearance of muon neutrinos. Because it was initially discovered in atmospheric neutrino experiments, it is sometimes known as the atmospheric mixing angle. It has been measured thus far to be consistent with the maximum possible value: $\sin ^{2} 2 \theta_{23}>0.91$.

- $\theta_{13}$, a still unknown parameter. It governs the probability that propagation involving the larger $\Delta \mathrm{m}_{13}^{2}$ associated with atmospheric neutrinos will involve electron flavor change. Its upper limit is $\sin ^{2} 2 \theta_{13}<0.15$.

- $\delta$, the parameter representing a phase that governs the CP-violating difference between neutrino and antineutrino flavor change; its value is completely unknown.

- The hierarchy, or ordering, of the neutrino masses is contained in the signs of the linear mass differences. The sign of $\Delta \mathrm{m}_{21}^{2}$ is known, so the sign of $\Delta \mathrm{m}_{32}^{2}$ completely determines this ordering. The latter sign, and therefore the overall hierarchy, is completely unknown.

\footnotetext{
${ }^{5}$ For comparison, the mass of the electron, the lightest of the leptons, is $0.511 \times 10^{6} \mathrm{eV}$.
} 
- The effects of matter that produce resonant MSW oscillations are contained in additional calculable parameters.

This picture of three elementary particles of very tiny mass evolving into and out of each other has the ring of science fiction. Indeed, it took some time and extraordinary evidence to be accepted by the scientific community. How is it known that neutrino oscillations do occur? The data summarized above were gathered by underground experiments of the type assessed in this report.

The first experimental indications that neutrinos oscillate came from the experiment in Homestake mine by Davis, followed by Kamiokande-II's direct detection of solar neutrinos and the other solar neutrino experiments with gallium, SAGE and GALLEX, which observed solar neutrinos from the fundamental proton-proton fusion reaction for the first time. The final incontrovertible evidence came from experiments at SNO that definitively confirmed changes in neutrino flavor. Meanwhile, measurements of neutrinos created in Earth's atmosphere (originally considered a background to proton decay experiments) at Super Kamiokande in 1998 showed that muon neutrinos arising from cosmic ray interactions in the atmosphere (so-called atmospheric neutrinos) disappear as a function of distance.

The study of antineutrinos from reactors has also been important, starting with the first observations of neutrinos in the 1950s by Reines and Cowan and leading through a long series of experiments to KamLAND. By observing electron antineutrino disappearance from all the reactors in Japan, this experiment eliminated any credible option to neutrino oscillations as explanations of solar and atmospheric neutrino effects.

Just as in more conventional particle physics, where initial observations of new particles in cosmic rays gave way to the controlled creation of new particles in accelerators, precision observations of neutrino oscillations must move from the now exploited natural sources to controlled high-flux accelerator-produced neutrinos. In this way, the $\mathrm{K} 2 \mathrm{~K}$ and MINOS experiments have already provided observations of muon neutrino disappearance, the NOVA experiment will operate in the next few years, and the new T2K experiments are just beginning to produce results in a search for electron appearance in a muon neutrino beam.

Our current knowledge of some of these neutrino oscillation parameters, arising from experiments performed to date, is briefly summarized in the above list. The mixing angles show a curious mix: Two are rather large and the third $\left(\theta_{13}\right)$ is currently consistent with zero. While the sign of the very small $\Delta \mathrm{m}_{21}^{2}$ has been determined by matter effects in the Sun, the sign of the other much larger masssquared difference $\left(\Delta \mathrm{m}_{32}^{2}\right)$ is unknown. The overall mass hierarchy is therefore unknown. The CP phase parameter, $\delta$, has not so far been determined by any 
experiment. Measurements of these three $\left(\theta_{13}, \delta\right.$, and the sign of $\left.\Delta \mathrm{m}_{32}^{2}\right)$ are major goals of future experiments.

\section{Four Critical Questions on the Nature of Neutrino Oscillations}

The above discussion leads to four critical questions on neutrino oscillations that could be addressed by the long-baseline neutrino oscillation experiment (LBNE) discussed in this report. These questions (or close variants of them) have also been discussed by other review bodies such as the DOE/NSF Neutrino Scientific Assessment Group (NUSAG) and the Particle Physics Project Prioritization Panel (P5), and by the NRC report Connecting Quarks to the Cosmos. They have motivated a number of related international projects such as T2K in Japan and LAGUNA-LBNO (proposed) in Europe. The committee agrees with those other bodies that these questions are among the highest priority questions in particle physics today, and longbaseline neutrino oscillation experiments are essential to answer them. No credible experimental alternatives exist that would not require large underground detectors. The four critical questions that need answers are these:

1. What is the value of the mixing angle $\theta_{13}$ ? Presently we only have limits on $\theta_{13}$. A null value, $\theta_{13}=0$, would point to a deeper symmetry. On the other hand, $\theta_{13} \neq 0$ would imply observable phenomena (questions 2 and 3) that answer other key neutrino oscillation questions.

2. What is the hierarchy of the neutrino masses? Is it similar to that in the quark sector, so that the neutrino mostly made up of the same flavor as the heaviest charged lepton is the heaviest neutrino ("normal" hierarchy)? Or is it the lightest ("inverted" hierarchy)? This hierarchy is determined by the sign of $\Delta \mathrm{m}_{31}^{2}$ and has important implications for both neutrino oscillations and neutrinoless double-beta decay, discussed in the next section.

3. Is CP violated in the neutrino sector and if so, what is the value of the phase $\delta$ ? This is a key question, since observing CP violation in neutrino oscillations would open a new window into the physics of matter and antimatter, providing essential inputs into models of leptogenesis, discussed more fully in the section on proton decay.

4. Are there new neutrino properties (or new neutrinos) that are not described by the three flavor neutrino model? Anomalies in existing data do not fit into this model, although no anomaly so far has been confirmed. ${ }^{6}$ However,

\footnotetext{
${ }^{6}$ Neutrino oscillation experiments are very difficult, often limited by systematic effects or backgrounds, and initially with only modest statistical precision. It is therefore essential that multiple observations be made with complementary techniques and with different energies, initial flavors, energies, and baselines.
} 
the history of neutrino physics is full of surprises, and the existence of a simple phenomenological model that works does not guarantee its correctness. Nature is often richer than imagined. New neutrino properties and new neutrino states ${ }^{7}$ could emerge if the neutrino model described here cannot fit all the observations.

\section{Experimental Details}

The amount of observable oscillation depends on the ratio of the distance between where the neutrinos are produced and where they are detected to the neutrino energy $(\mathrm{L} / \mathrm{E})$. For ranges of neutrino energies that are easily produced and detected with present technology, a detector must be located appropriately. On the one hand, if it is placed too far from the source, it sees little flux and presents technical difficulties in beam construction (because of Earth's curvature). On the other hand, a minimum distance ("baseline") of about 1,000 km is needed in order to provide sufficient time and distance for the neutrinos to oscillate. Such an experiment requires an intense neutrino source, as well as a massive and sensitive detector. In order to eliminate backgrounds from cosmic ray events, it must be located underground. An experiment with such capabilities—-the LBNE—will allow, in addition to the search for $\mathrm{CP}$ violation, a broad program of neutrino physics, as well as sensitivity to proton decay.

The Homestake site, the intended location for the DUSEL program, is approximately $1,250 \mathrm{~km}$ from Fermilab, the presumptive neutrino source. The principal existing general science underground laboratory, the Soudan Underground Laboratory, is only $730 \mathrm{~km}$ from Fermilab. Other sites considered in the DUSEL site selection process that culminated in choosing Homestake also meet the requisite minimum 1,000 km distance from Fermilab. Similar large-scale long-baseline experiments are under consideration in Japan and Europe. The baseline length between J-PARC and Kamioka in Japan is $295 \mathrm{~km}$, which is too short for the determination of mass hierarchy. Furthermore, the $\mathrm{CP}$ violation parameter cannot be determined uniquely with this configuration alone due to the immeasurable mass hierarchy. In Europe, studies are in progress to select a possible underground site for future large detectors. The physics questions that can be addressed by such detectors will depend on the selected site and detector technology.

If LBNE proceeds, the design and construction of it and the neutrino beamline will take at least 7 years. To consider what new knowledge LBNE would provide requires a comparison with the expectations of experiments currently operating or under construction. Experiments that will be sensitive to electron neutrino

\footnotetext{
${ }^{7}$ Such new states are called sterile neutrinos, because other measurements show that only the three currently known neutrino species can have normal weak interactions.
} 
appearance on that timescale include the long-baseline experiments MINOS, T2K, and NOvA and the reactor experiments RENO, Double Chooz, and Daya Bay. The last five are focused primarily on determining $\theta_{13}$. Their sensitivity to $\theta_{13}$ as a function of time is difficult to predict precisely, but by 2020 they are expected to have measured a finite value if $\sin ^{2} 2 \theta_{13}$ is greater than $0.03-0.04$. By comparing the results from these experiments, it will be possible to place constraints on the other oscillation parameters. In particular, combined results from these experiments could provide some evidence for CP violation over about 20 percent of the allowed parameter space if $\sin ^{2} 2 \theta_{13}$ is in this range. Even in the most optimistic scenarios, the statistical significance of such a result would be marginal. In addition, experimental data obtained before the LBNE becomes operational would have only a small window for determining whether the mass hierarchy is inverted or normal.

LBNE therefore offers the real prospect of a transformative discovery of CP violation in the lepton sector, with sensitivity greater than three sigma over half the possible values of $\delta$ for $\sin ^{2} 2 \theta_{13}$ greater than 0.03 after 10 years of operation at the initial beam intensity. ${ }^{8}$ With potential future accelerator upgrades, sensitivity to values of $\theta_{13}$ extends to almost an order of magnitude below expected preLBNE limits. In addition, for $\sin ^{2} 2 \theta_{13}$ greater than 0.04 , LBNE can unambiguously distinguish the normal from the inverted hierarchy over the full range of possible CP parameters. Determination of the hierarchy would shed some light on whether neutrinos have the same flavor-ordering of masses and perhaps demonstrate that the source of neutrino mass is different from the source of mass for other leptons and quarks. It would also significantly impact the interpretation of the sensitivity of any double-beta decay experiments.

The main goal of LBNE is to significantly extend our sensitivity to the neutrino oscillation parameters over existing experiments using a broad-band neutrino beam (a beam with a wide range of neutrino energies) with a peak energy of about $3.5 \mathrm{GeV}$ from Fermilab. The experiment requires a small "near detector" located at the Fermilab site and a much more massive "far detector" located underground. Both detectors would observe the flux of neutrinos of a given flavor by reconstructing neutrino interactions through charged current processes that identify the final-state charged-lepton flavor. The near detector would monitor the flux and composition of the neutrino beam near the point of production. The far detector would primarily search for the appearance of electron neutrinos (or antineutrinos) in the muon neutrino (or antineutrino) beam. The details of the oscillation predictions are complex because it is necessary to include the interference effects of three-flavor mixing as well as the effects of neutrino interactions with the portions of Earth traversed by the beam. It is precisely these interference effects that produce a potentially observable CP violation effect; however, they

\footnotetext{
${ }^{8}$ http://www.int.washington.edu/PROGRAMS/10-2b/LBNEPhysicsReport.pdf.
} 
make the oscillation probability at any particular baseline and neutrino energy depend on all the oscillation parameters. Thus the oscillations must be observed over an extended energy range for both neutrinos and antineutrinos in order to disentangle all the parameters.

Two main technologies for the far detector have been proposed. The first entails building a huge water Cherenkov detector similar in design to the extremely successful Super-Kamiokande experiment in Japan, but larger by a factor of eight. Energetic charged particles traveling through a transparent medium such as water emit a cone of Cherenkov light until they slow down below the speed of light in water. A water Cherenkov detector consists of a large tank of water with the vessel surface partially covered by inward-looking photomultiplier tubes (PMTs), large sensors capable of detecting single photons of Cherenkov light. Each charged particle appears as a ring of light detected by the PMTs, with electrons distinguished from muons by the sharpness of the ring (electrons undergo much more multiple scattering in water than muons, and so have fuzzier rings). Water Cherenkov detectors are a well-proven and well-understood technology. However, since they cannot detect slow particles and because rings can be merged or confused in events with many charged particles, they have a relatively low efficiency for low-velocity particles, on the one hand, and a significant fraction of misreconstructed events for the relatively complicated events in the multi-GeV range.

The alternative technology is a liquid argon (LAr) tracking calorimeter similar in concept to the ICARUS detector currently in the Laboratori Nazionali del Gran Sasso in Italy, but larger by a factor of 40. In a LAr detector, ionization deposited along charged particle tracks is drifted to a grid of sense wires, allowing the tracks to be reconstructed in three dimensions. Such a detector is sensitive to low-velocity particles, and the spatial resolution is excellent (potentially a few millimeters or even better). Thus a LAr detector is capable in principle of reconstructing quite complex events and is expected to have a lower misreconstruction fraction and higher efficiency. Because of this added efficiency, a LAr detector can be smaller in mass by a factor of about six and (owing to the greater density of LAr) almost an order of magnitude smaller in volume. As a result, the far detector hall could be much smaller than for the other option. However, there is much less experience with large LAr neutrino detectors than with water Cherenkov detectors. The challenges include the technical complexity and safety considerations involved in producing and retaining multikiloton volumes of a cryogenic noble liquid in an underground laboratory, the high purity requirements for the argon, and the technical complexity of the readout system. Techniques for reconstructing LAr events are still in development. The development and operation of the MicroBOONE 800-ton LAr experiment at Fermilab will be a first step in resolving many of these technical issues.

From the point of view of neutrino oscillations alone, the physics sensitivity of the water Cherenkov module and the LAr options are similar. The choice 
between them relies heavily on technical and financial considerations. The choice of technology may also affect the required depth underground. This requirement is under study and has not yet been firmly established.

The first observation of CP violation in the neutrino sector will be followed by a long sequence of experiments of different types intended to more accurately measure the oscillation parameters and to understand whether the three-neutrino parameterization is correct. There are at present uncorroborated anomalies that, if correct, would need to be explained through modifications or additions to this picture.

The two technologies discussed above have different and complementary strengths both for the initial discovery of nonstandard phenomena and for second-generation measurements. Proposals for new second-generation experiments with water Cherenkov detectors include very imaginative possibilities, such as the DAEdALUS proposal to create neutrinos using a series of small nearby cyclotrons. These may well become important complementary techniques and illustrate the possibilities for additional use of a large water Cherenkov detector for constraining neutrino parameters, depending on what is found. Liquid argon, on the other hand, should be able to analyse complicated events with its particle identification and tracking capabilities, which may also open new possibilities.

LBNE would also allow a broad program of physics measurements beyond accelerator-produced neutrinos. Examples include studies of atmospheric neutrinos, solar neutrinos, and neutrinos from astrophysical sources.

There is a long history of measurements of atmospheric neutrinos that will continue over the next decade with new results from the Super-Kamiokande detector. With the same kind of water Cherenkov detector, the statistical improvement would be at best nominal, by a factor of, say, 2 or 3. Although an alternative LAr detector of lower tonnage would provide less statistical improvement, it might be able to make a more definitive observation of tau neutrinos produced from oscillated cosmic ray muon neutrinos. This would depend on many factors not yet demonstrated.

A massive water Cherenkov detector would allow measurements with high statistics of the small day-night asymmetry in electron solar neutrino flux that arises from the small ( $\sim 2$ percent) additional oscillation that takes place within the matter of Earth. Thus far, Super-Kamiokande has measured only a small (negligible) effect (2 standard deviations). A LAr detector would have better particle detection and identification for much less tonnage. In any case, use of LAr for solar neutrinos would require addressing significant technical issues, including the production of ${ }^{39} \mathrm{Ar}$ by cosmic rays, a source of serious background signal, as well as control of radon. These technical issues mean that solar measurements would probably require substantial depth. 


\section{Summary}

Realization of an LBNE with experimental reach for CP violation and the understanding of the mass hierarchy will require a large underground detector and a high-intensity neutrino source separated from each other by more than 1,000 km or so. Such an experiment has the potential to determine whether the current phenomenological description of neutrino oscillations is correct and to measure the associated parameters. Observations of both the mass hierarchy and CP violation in the neutrino sector would have profound effects on extensions to the Standard Model as well as on our ability to model the early Universe. An experiment capable of these discoveries will enable a broad program of discovery and measurement in neutrino physics. Such a program would be a cornerstone of basic science research in the United States.

Conclusion: The long-baseline neutrino oscillation experiment is of paramount scientific importance and will address crucial questions upon whose answers the tenets of our understanding of the Universe depend. This experiment would not only provide an exceptional opportunity to address scientific questions of paramount importance, it would also have a significant positive impact upon the stewardship of the particle physics and nuclear physics research communities and have the United States assume a visible leadership role in the expanding field of underground science. The U.S. particle physics program is especially well positioned to build a world-leading long-baseline neutrino experiment due to the combined availability of an intense neutrino beam from Fermilab and a suitably long baseline from the neutrino source to an appropriate underground site such as the proposed DUSEL.

\section{The Nature of Neutrinos-Antiparticles, Mass Scale} (Neutrinoless Double-Beta Decay)

In 1937, the Italian physicist Ettore Majorana conjectured that the neutrino could be its own antiparticle, thereby lending his name to particles that have this characteristic of being their own antiparticle. ${ }^{9}$ Whether or not neutrinos are Majorana particles remains a fundamental and unresolved question in particle and nuclear physics. Double-beta decay experiments could resolve this question.

Double-beta decay is a process in which a nucleus decays into another nucleus with the same mass and two more protons by emitting two electrons. Because it

\footnotetext{
${ }^{9}$ E. Majorana. 1937. Nuovo Cimento 14: 171.
} 


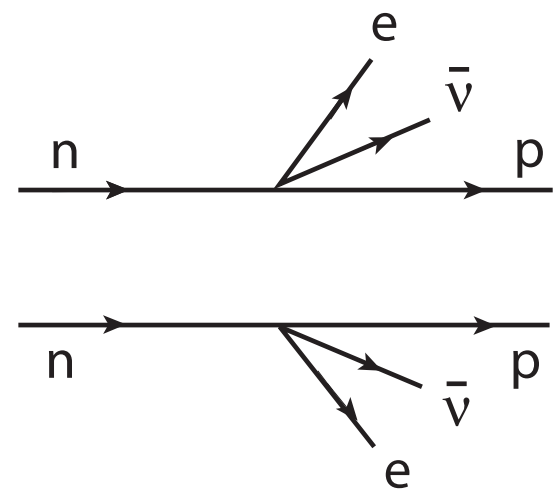

(a)

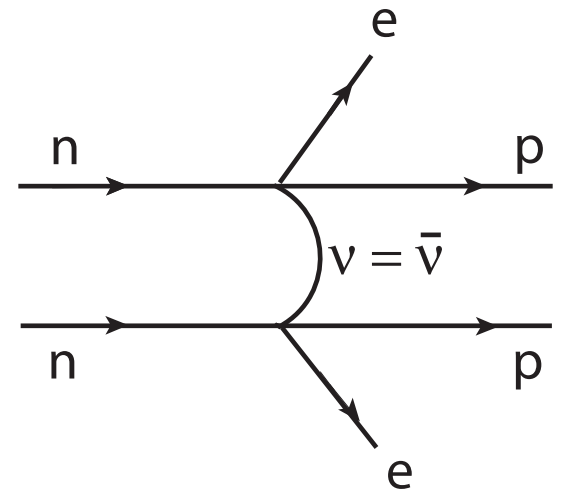

(b)

FIGURE 3.4 Feynman diagrams describing double-beta decay processes in which two neutrons $(n)$ in a nucleus are converted to two protons (p): (a) $2 v \beta \beta$, in which two antineutrinos $(\bar{v})$ are emitted; (b) $0 v \beta \beta$, in which no neutrinos are emitted. This second process requires that the neutrino is its own antiparticle $(v=\bar{v})$. SOURCE: Courtesy of Yoram Alhassid, Yale University.

typically is accompanied by the emission of two electron antineutrinos, it is known as two-neutrino, double-beta $(2 v \beta \beta)$ decay (see Figure $3.4 a)$. In the absence of emitted neutrinos, the process is called neutrinoless double-beta $(0 \mathrm{v} \beta \beta)$ decay ${ }^{10}$ (see Figure $3.4 \mathrm{~b}$ ). The $2 v \beta \beta$ decay can occur whether or not the neutrino is a Majorana particle and has been observed in a number of nuclei. However, the $0 \mathrm{nbb}$ decay can occur only if the neutrino is a Majorana particle, so its existence would be an unambiguous demonstration of the neutrino's peculiar nature. Thus far, no confirmed observation of such a decay has been made.

Establishing that neutrinos are Majorana particles would have a number of important consequences. Because 0v $\beta \beta$ decay rates depend on neutrino masses, determining those rates would be the most sensitive laboratory experiment to determine the neutrino mass scale. The $0 v \beta \beta$ decay rate is calculated to be proportional to the square of an effective neutrino mass and a quantity that is determined by the nuclear structure of the corresponding decaying nucleus. Various nuclear structure models have been used to calculate this quantity and, with recent progress, they agree with each other to within a factor of about two. The observation of a Majorana neutrino would also provide support for a subset of GUTs that have massive Majorana neutrinos.

\footnotetext{
${ }^{10}$ For a recent review, see F.T. Avignone III, S.R. Elliott, and J. Engel. 2008. Review of Modern Physics 80: 481 and references therein.
} 
Although the values of the neutrino masses are not known precisely, neutrino oscillation studies and other evidence indicate they are much smaller (by factors of many millions) than those of other elementary particles, whose masses are thought to be generated by the well-known Higgs mechanism. This fact alone strongly indicates that neutrino masses are due to very different and very likely much higher energy mechanisms. In many GUTs, such a mechanism is most natural if the neutrino is its own antiparticle-that is, if it is a Majorana particle.

Finally, the $0 v \beta \beta$ decay implies a change of lepton number by two units, and its observation would lead to the important conclusion that lepton number conservation is violated. This provides support to leptogenesis, a process that violates CP and lepton number conservation and leads to lepton-antilepton asymmetry. In turn, this lepton-antilepton asymmetry could lead to baryonantibaryon asymmetry and might help to explain the preponderance of matter over antimatter in the Universe (see discussion in the section "Tests of Grand Unification Theories").

However, even if $0 v \beta \beta$ decay is not observed, such studies can provide important information about the nature and mass of neutrinos. The neutrino oscillation data are consistent with the three neutrinos having different masses and two of the masses having a smaller mass splitting as determined by the solar mass scale. However, there are still two possibilities for the neutrino spectrum: the normal hierarchy and the inverted hierarchy (see discussion in the section "The Nature of Neutrinos-Oscillations [Long-Baseline Neutrino Experiment]"). If oscillation experiments demonstrate that the mass hierarchy of neutrinos is inverted, then having $0 v \beta \beta$ decay results establish an upper limit of $20 \mathrm{meV}$ on the effective Majorana neutrino mass would show that neutrinos are not Majorana particles (see Figure 3.5). Third-generation experiments at the ton scale would reach this limit, so that the failure to observe $0 v \beta \beta$ decay would rule out neutrinos being a Majorana particle in the inverted hierarchy scenario. In the case of the normal hierarchy scenario, an experimental sensitivity of $1 \mathrm{meV}$ would likely be required to rule out most possibilities for a Majorana neutrino. Furthermore, an experimental lifetime limit for the $0 v \beta \beta$ decay will directly constrain the mass of the lightest neutrino (assuming the neutrino is a Majorana particle).

\section{Two Critical Questions on the Nature of Neutrinos-Antiparticles and Mass Scale}

The above discussion leads to two critical discussions that could be addressed by the neutrinoless double-beta decay experiment considered in this report.

- Are neutrinos Majorana or Dirac particles? It would be an amazing discovery in itself to demonstrate the existence of an entirely new type of elementary particle: one that is its own antiparticle. However, the existence 
of Majorana neutrinos also allows leptogenesis to be an explanation for the matter-antimatter asymmetry of the Universe.

- What is the absolute mass scale of neutrinos? Knowledge of the absolute mass scale is needed in order to understand neutrino masses within the framework of particle physics, as well as to gauge the impact that massive neutrinos have on cosmology. Neutrino oscillation experiments cannot determine the absolute mass scale (only the squared mass differences), but neutrinoless double-beta decay experiments address this question.

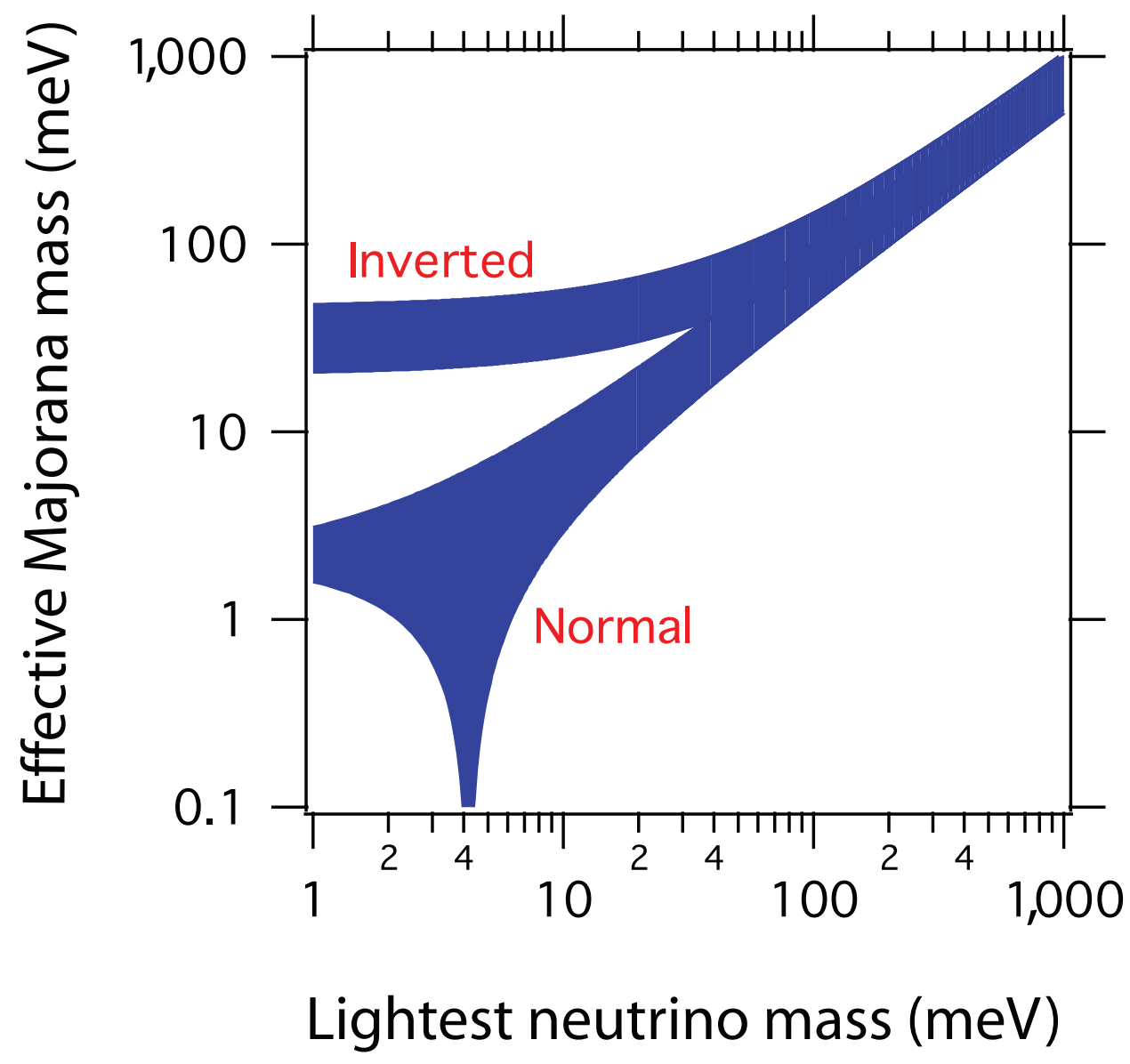

FIGURE 3.5 Relation between the effective Majorana mass and the lightest neutrino mass. Both inverted and normal mass hierarchies are considered. SOURCE: Reprinted with permission from F.T. Avignone, S.R. Elliott, and J. Engel. Double beta decay, Majorana neutrinos, and neutrino mass. Reviews of Modern Physics 80: 481. Copyright 2008 by the American Physical Society. 


\section{Experimental Aspects}

There is an overwhelming interest in the international particle and nuclear physics communities to pursue the science of $0 v \beta \beta$ decay. Many of the underground laboratories have programs to search for the process, including Gran Sasso in Italy, Canfranc in Spain, Modane in France, Kamioka in Japan, SNOLAB in Canada, and the Waste Isolation Pilot Plant (WIPP) and Sanford Underground Laboratory in the United States. A number of the offshore experiments have significant U.S. involvement.

The typical $0 v \beta \beta$ decay experiment consists of a set amount of an isotope susceptible to double-beta decay in which detectors have been incorporated and which involves searching for very rare monoenergetic electron signals superimposed on continuum backgrounds. Because cosmic ray muons can create neutrons whose interactions form such a continuum, experiments must be conducted deep underground where such muons only rarely penetrate. A reliable $0 v \beta \beta$ decay program requires multiple experiments worldwide using different isotopes. There are several reasons for this: (1) very different experimental techniques are used for different isotopes, some of which may prove to be more effective in, for example, background suppression; (2) a signal observed in one particular isotope might be a misidentification because of unknown background; (3) if a signal is detected, the measurement of multiple isotopes can provide a more reliable effective neutrino mass given the uncertainties in the calculated nuclear matrix elements; and (4) measuring the signal in different isotopes can help distinguish between different possible mechanisms of $0 v \beta \beta$ decay. Worldwide, there are ongoing or proposed experiments searching for $0 v \beta \beta$ in ${ }^{48} \mathrm{Ca},{ }^{76} \mathrm{Ge},{ }^{82} \mathrm{Se},{ }^{100} \mathrm{Mo},{ }^{116} \mathrm{Cd},{ }^{130} \mathrm{Te},{ }^{136} \mathrm{Xe},{ }^{150} \mathrm{Nd}$, and ${ }^{160} \mathrm{Gd}$. Those experiments use several key experimental detection techniques, such as calorimetric bolometers (e.g., CUORICINO and CUORE at Gran Sasso), cryogenic semiconductor detectors (e.g., GERDA at Gran Sasso and MAJORANA at the Sanford Underground Laboratory), and liquid/gas detectors (e.g., SNO+ at SNOLAB and EXO at WIPP). See Table 3.2 for a more complete list.

Since the $0 v \beta \beta$ decay is a very rare process, large masses of the corresponding isotope are required to reach a given sensitivity. First-generation (G1) experiments use detector masses in the range of $10-25 \mathrm{~kg}$ and have sensitivity to a neutrino mass of about $1 \mathrm{eV}$. Typically, these are prototype experiments to demonstrate the feasibility of various techniques. Demonstrating the scalability of a particular method is accomplished by using 30-200 kg detectors, which provide sensitivities down to $100 \mathrm{meV}$. There are about 10 of these second-generation (G2) experiments, and all experiments currently running or in construction are either G1 or G2 experiments.

Reaching the atmospheric scale (the mass scale associated with atmospheric muon neutrino disappearance) of $50 \mathrm{meV}$ requires third-generation (G3) detectors using detectors with masses of 1 ton or more. For the reasons above, a meaningful 
TABLE 3.2 Ov $\beta \beta$ Decay Experiments Worldwide Classified by Generation and Experimental Technique

\begin{tabular}{|c|c|c|c|c|}
\hline \multirow[t]{2}{*}{ Generation } & \multicolumn{4}{|c|}{ Experimental Techniques } \\
\hline & $\begin{array}{l}\text { Calorimetric } \\
\text { Bolometer }\end{array}$ & $\begin{array}{l}\text { Calorimetric } \\
\text { Semiconductor }\end{array}$ & $\begin{array}{l}\text { Calorimetric } \\
\text { Liquid/Gas }\end{array}$ & $\begin{array}{l}\text { Tracking } \\
\text { Calorimetry }\end{array}$ \\
\hline G1 & $\begin{array}{l}\text { CUORICINO, } \\
\text { Gran Sasso }\end{array}$ & $\begin{array}{l}\text { Heidelberg-Moscow, } \\
\text { Gran Sasso }\end{array}$ & & $\begin{array}{l}\text { NEM03, } \\
\text { Modane }\end{array}$ \\
\hline \multirow[t]{4}{*}{ G2 } & $\begin{array}{l}\text { CUORE, } \\
\text { Gran Sasso }\end{array}$ & $\begin{array}{l}\text { GERDA-I-II, } \\
\text { Gran Sasso }\end{array}$ & $\begin{array}{l}\text { XMASS, } \\
\text { Kamioka }\end{array}$ & $\begin{array}{l}\text { SuperNEMO, } \\
\text { Modane }\end{array}$ \\
\hline & $\begin{array}{l}\text { CANDLE, } \\
\text { Kamioka }\end{array}$ & $\begin{array}{l}\text { Majorana Demonstrator, } \\
\text { Sanford Lab }\end{array}$ & $\begin{array}{l}\text { SNO+, } \\
\text { SNOLab }\end{array}$ & \\
\hline & $\begin{array}{l}\text { LUCIFER, } \\
\text { Gran Sasso }\end{array}$ & $\begin{array}{l}\text { COBRA, } \\
\text { Gran Sasso }\end{array}$ & $\begin{array}{l}\text { EXO-200, } \\
\text { WIPP }\end{array}$ & \\
\hline & & & $\begin{array}{l}\text { NEXT, } \\
\text { Canfranc }\end{array}$ & \\
\hline \multirow[t]{2}{*}{ G3 } & & $\begin{array}{l}\text { GERDA-III, } \\
\text { Gran Sasso }\end{array}$ & $\begin{array}{l}\text { XMASS-10 ton, } \\
\text { Kamioka }\end{array}$ & \\
\hline & & $\begin{array}{l}\text { Majorana, } \\
\text { Sanford Lab }\end{array}$ & & \\
\hline
\end{tabular}

NOTE: The G1 experiments use on the order of $10 \mathrm{~kg}$ of isotopes. Most projects plan on scaling up the corresponding isotope mass (and sensitivity) by a factor of $10(\mathrm{G} 2)$ during the next 5 years and moving up to the ton scale (G3) within 10 years. The latter can probe most of the phase space permitted by the inverted hierarchy spectrum of neutrino masses.

0v $\beta \beta$ decay program requires multiple experiments and, although costly, there should be at least two such G3 experiments worldwide. It is appropriate that such a 1-ton detector be mounted at a U.S. site: (1) a $0 v \beta \beta$ decay experiment in this country will be part of the required complement of experiments worldwide using different isotopes and different techniques, (2) a detector installed at a U.S. facility will ensure U.S. leadership in this field and enable U.S. scientists to participate more readily, and (3) a U.S. facility hosting a ton-scale $0 v \beta \beta$ decay detector will attract top foreign scientists in the field and will foster international collaborations. At the same time, U.S. scientists are likely to continue their involvement in $0 v \beta \beta$ decay experiments abroad.

The primary technical challenge facing the $0 v \beta \beta$ experiments is to increase their scale at reasonable costs. A list of $0 v \beta \beta$ decay experiments around the world is provided in Table 3.2. The experiments are tabulated according to their generation and the experimental technique they use. Proponents of these experiments have made convincing cases that at least the xenon and germanium experiments can scale to 1 ton. Going to the even larger detector masses needed to test limits for the normal mass hierarchy will present a more difficult challenge. 
To reach sensitivity at the solar scale (the mass scale associated with the solar electron neutrino disappearance) of $5 \mathrm{meV}$ and even down to $1 \mathrm{meV}$ requires a 50 -ton detector. Such a massive $0 v \beta \beta$ detector is difficult to contemplate: A 50 -ton germanium detector would be the size of a small house and very expensive. However, technology does advance, and it makes sense to ensure that the underground cavern for a $0 v \beta \beta$ experiment could eventually accommodate a 50-ton experiment and its attendant shielding.

Detectors in the mass range of 10-25 kg have established the current limits on ov $\beta \beta$ decay lifetime to be around $10^{25}$ years, implying that the effective Majorana neutrino mass scale must be less than $1 \mathrm{eV}$. There is one claimed observation of $0 v \beta \beta$ decay in ${ }^{76} \mathrm{Ge}$ with a lifetime of $2 \times 10^{25}$ years, ${ }^{11}$ but the interpretation of the data is disputed. A potential scientific challenge to extracting an effective neutrino mass from the $0 v \beta \beta$ decay measurements is the numerical uncertainty in the nuclear structure calculations. There has been much theoretical progress in improving these calculations in the last few years. Further progress is likely, and should minimize the nuclear structure uncertainties on the effective neutrino mass derived for the timescale of much larger experiments.

\section{Summary}

The $0 v \beta \beta$ experiment addresses crucial unanswered questions in particle and nuclear physics. ${ }^{12}$ It is the only practical experiment that could determine whether the neutrino is a Majorana or Dirac particle. If the neutrino is a Majorana particle, it would also be the most sensitive laboratory experiment that could measure or at least constrain the absolute mass scale of neutrinos. Were $0 v \beta \beta$ to be observed, it would tell us that neutrinos are Majorana particles and lepton number conservation is violated, a model-independent conclusion and a Nobel Prize-level achievement. The $0 v \beta \beta$ decay is very rare and its detection necessitates massive detectors. Given the paramount scientific importance of this experiment and the leadership roles taken by U.S. scientists, it is appropriate that the United States take a leadership role in one $0 v \beta \beta$ decay ton-scale experiment for installation at a U.S. site, or at an appropriate foreign facility if necessary, and that U.S. scientists be supported to participate in other such experiments worldwide.

Conclusion: The neutrinoless double-beta decay experiment, like the direct detection dark matter experiment and the long-baseline neutrino oscillation experiment, is of paramount scientific importance and will address crucial

\footnotetext{
${ }^{11}$ H.V. Klapdor-Kleingrothaus, A. Dietz, L. Baudis, et al. 2001. European Physics Journal A 12: 147.

${ }^{12}$ NRC. 2006. Revealing the Hidden Nature of Space and Time. Washington, D.C.: The National Academies Press, p. 13.
} 
questions upon whose answers the tenets of our understanding of the Universe depend. These three experiments are of comparable scientific importance. This experiment would not only provide an exceptional opportunity to address scientific questions of paramount importance, it would also have a significant positive impact upon the stewardship of the particle physics and nuclear physics research communities and would have the United States assume a visible leadership role in the expanding field of underground science. In light of the leading roles played by U.S. scientists in the study of neutrinoless double-beta decay, together with the need to build two or more large experiments in this area, U.S. particle and nuclear physicists are also well positioned to assume leadership roles in the development of one neutrinoless double-beta decay experiment on the scale of a ton. While installation of such a U.S.-developed experiment in an appropriate foreign facility would significantly benefit scientific progress and the research communities, there would be substantial advantages to the communities if this experiment could be installed within the United States, possibly at the same site as the longbaseline neutrino experiment.

\section{Proton Decay}

\section{Overview}

Atoms are made of electrons and nuclei and nuclei are made of protons and neutrons. Protons and neutrons are the lightest particles that carry the baryon number, B, a quantum number that is conserved in Standard Model processes. While free neutrons, slightly heavier than protons, decay to protons with a lifetime of about $900 \mathrm{~s}$, they can live much longer when bound in nuclei. The free neutron decays through nuclear beta decay, a weak interaction $\left(\mathrm{n} \rightarrow \mathrm{p}+\mathrm{e}^{-}+\bar{v}_{\mathrm{e}}\right.$, where $\mathrm{n}$ is the neutron, $\mathrm{p}$ is a proton, $\mathrm{e}^{-}$an electron, and $\bar{v}_{\mathrm{e}}$ an electron antineutrino). Experimentally, there is no evidence that the proton, as the lightest baryon, decays at all. However, the stability of the proton is a very fundamental question and observation of proton decay would constitute a major scientific discovery. GUTs predict proton decay although the lifetime of the proton would be very long, and decay rate predictions are sensitive to a broad choice of theoretical model and the parameters of that model, leaving little theoretical guidance to the expected lifetime or the dominant decay mode. In practice, observation of proton decay would provide essential input to GUT models. In this situation, new experiments must be evaluated based on their "reach" - that is, on how far they can extend present sensitivity.

Because the lifetime of the proton is so long, the probability of seeing any individual proton decay is very small. Discovering proton decay becomes possible 
only if a large number of protons are observed; thus, proton decay detectors must be massive. Since size and many of the other requirements for a successful proton decay experiment coincide with those of the LBNE, it is expected that the nextgeneration long-baseline neutrino detector will also serve as a next-generation proton decay detector.

\section{Scientific Landscape}

In the original GUT of Georgi and Glashow, the prediction for the lifetime of the proton was $4.5 \times 10^{(29 \pm 1.7)}$ years, and the main decay mode was predicted to be $\mathrm{p} \rightarrow \mathrm{e}^{+}+\pi^{0}$. The prediction for the rate was highly uncertain as it was proportional to the fourth power of the unification scale (the energy scale above which the electromagnetic, weak, and strong forces are equal in strength). Current bounds on the proton lifetime set by experiment decisively rule out this original model. That model had several other shortcomings. In particular, it did not address the "hierarchy problem," the issue of why the weak and grand unified energy scales differ by 12 orders of magnitude. It also made other predictions that were ruled out by experiments.

Subsequent GUT theories introduce the concept of supersymmetry (a symmetry between bosons and fermions often referred to as SUSY), which plays an important role in addressing the hierarchy problem. Other SUSY predictions were consistent with experiment, especially for the so-called weak interaction mixing angle. Supersymmetry raised the unification scale by a factor of 100 , and so this class of theories increases the predicted lifetime for the decay $\mathrm{p} \rightarrow \mathrm{e}^{+}+\pi^{0}$ by as much as eight orders of magnitude, well beyond experimental reach. With SUSY, however, new modes of proton decay involving kaons can become important. The minimal supersymmetric GUT predicts a dominant proton decay mode to be $\mathrm{p} \rightarrow \mathrm{K}^{+}+\bar{v}$, where $\mathrm{K}^{+}$is a kaon and $\bar{v}$ an antineutrino, with a lifetime prediction as small as $10^{32}$ years. (This prediction reduces the dependence on the masses of GUT-scale particles from the fourth power to the square.) The prediction, however, is sensitive to unknown masses of weak-scale SUSY particles, as well as to uncertain details of the GUT. There are a large number of GUT models, as well as models that directly unify the forces of the Standard Model with quantum gravity in string theory models. However, all unified models predict that the proton does decay, and proton decay remains the main unverified prediction of unification, which has other successes. These successes include agreement with the measured weak interaction mixing angle discussed above and, importantly, the presence of small but nonzero neutrino masses and of neutrino oscillations. Detection of proton decay would unambiguously signal physics beyond the Standard Model. It would provide a guide to which of the many theoretical extensions of the Standard Model 
are worth pursuing, and it would provide crucial information about the origin of matter in the Universe.

The best current bounds on many nucleon decay rates come from the SuperKamiokande experiment. Water Cherenkov detectors, such as Super-Kamiokande, are highly efficient for the $\pi^{0} \mathrm{e}^{+}$mode. Decay modes containing kaons are more difficult, since the kaon is below the Cherenkov threshold and so can only be detected with reduced efficiency or much more stringent cuts. For this reason, limits on these modes are weaker. Present limits on decays with kaons are obtained by combining several such techniques. One example is the decay of a proton in an ${ }^{16} \mathrm{O}$ nucleus, detected with an efficiency of 11 to 13 percent by a coincidence between the subsequent kaon decay $\left(\mathrm{K}^{+} \rightarrow \mu^{+} v\right.$ or $\left.\mathrm{K}^{+} \rightarrow \pi^{+} \pi^{\circ}\right)$ with a $\gamma$ from de-excitation of the excited ${ }^{15} \mathrm{~N}$. Backgrounds for some modes of proton decay have been demonstrated to be very low at Super-Kamiokande; consequently, its bounds are expected to continue to improve, as they depend primarily on integrated exposure.

If LAr is the technology of choice for the LBNE detector, significant improvements to limits for proton decays to kaons could be possible. Since LAr allows for position resolutions of a few millimeters, complex events can be reconstructed in detail, with particle identification from energy loss and with photons distinguishable from electrons by the gap from the vertex and by ionization before a shower develops. However, since a LAr LBNE detector is expected to be less massive than a water Cherenkov detector, it would be less sensitive to those modes for which water Cherenkov detectors have high efficiency.

The impact of depth underground on background rates has been studied, ${ }^{13}$ leading to the conclusion that with an active muon veto shield, a proton decay search may be viable at fairly shallow depths for both water Cherenkov and LAr detectors. However, for the $\mathrm{p} \rightarrow \mathrm{K}^{+}+\bar{v}$ mode, expected background due to cosmic ray interactions in the rock near the detector can be eliminated only by limiting the fiducial volume to the central region of the detector volume. In order to keep the large fiducial volume, both the water Cherenkov and LAr detectors are recommended to be located deeper than 3,000 meters of water equivalent (m.w.e.).

Table 3.3 lists a few specific modes, theoretical expectations, lifetime bounds, ${ }^{14}$ the most sensitive experiments providing those bounds, and projected sensitivities of future LBNEs. The expectations for Super-Kamiokande in 2030, as well as the detector capabilities for configurations in scenarios wherein 10 years of data taking could have occurred on about the same timescale, are taken from the LBNE proton

\footnotetext{
${ }^{13}$ A. Bernstein, E. Blucher, D. Cline, et al. 2008. Report on the depth requirements for a massive detector at Homestake," available at http://www.bnl.gov/isd/documents/43873.pdf. Last accessed on September 22, 2011.

${ }^{14}$ From K. Nakamura, K. Hagiwara, K. Hikasa, et al. 2010. Review of particle physics, Journal of Physics G-Nuclear and Particle Physics 37: 075021. Available at http://iopscience.iop.org/09543899/37/7A/075021/media/rpp2010_0001-0007.pdf. Last accessed on September 22, 2011.
} 
TABLE 3.3 Current Limits on Lifetimes (Column 3) for a Few Proton Decay Modes and the Major Experiments Establishing Those Limits

\begin{tabular}{|c|c|c|c|c|c|c|}
\hline $\begin{array}{l}\text { Decay } \\
\text { Mode }^{a}\end{array}$ & $\begin{array}{l}\text { Predicted } \\
\text { Rate in } \\
\text { Various } \\
\text { Unified } \\
\text { Models }^{b}\end{array}$ & $\begin{array}{l}\text { Current } \\
\text { Bound on } \\
\text { Lifetime }^{a}\end{array}$ & $\begin{array}{l}\text { Main } \\
\text { Experiment } \\
\text { Setting } \\
\text { Current } \\
\text { Bound }^{c}\end{array}$ & $\begin{array}{l}\text { Expected } \\
\text { Super-K } \\
\text { Bound on } \\
\text { Lifetime by } \\
2030^{d}\end{array}$ & $\begin{array}{l}\text { LBNE } \\
\text { Sensitivity } \\
\text { for } 200 \\
\text { kT Water } \\
\text { Cherenkov } \\
\text { Detector after } \\
10 \text { Years }^{d}\end{array}$ & $\begin{array}{l}\text { LBNE } \\
\text { Sensitivity } \\
\text { for } 28 \text { kT } \\
\text { Liquid Argon } \\
\text { Detector after } \\
10 \text { Years }^{d}\end{array}$ \\
\hline$p \rightarrow \pi^{0} \mathrm{e}^{+}$ & $10^{(34-39)} \mathrm{y}$ & $8.2 \times 10^{33} y$ & Super-K & $3 \times 10^{34} y$ & $6.2 \times 10^{34} y$ & $1.0 \times 10^{34} y$ \\
\hline $\mathrm{p} \rightarrow \mathrm{K}^{+} \bar{v}$ & $10^{(33-39)} \mathrm{y}$ & $2.3 \times 10^{33} y$ & Super-K & $6 \times 10^{33} y$ & $12 \times 10^{33} y$ & $35 \times 10^{33} y$ \\
\hline$p \rightarrow \pi^{0} \mu^{+}$ & $10^{(34-39)} \mathrm{y}$ & $6.6 \times 10^{33} y$ & Super-K & & & \\
\hline$p \rightarrow e^{+} \gamma$ & $10^{(36-41)} \mathrm{y}$ & $6.7 \times 10^{32} y$ & IMB & & & \\
\hline$p \rightarrow \mu^{+} \gamma$ & $10^{(36-41)} \mathrm{y}$ & $4.8 \times 10^{32} y$ & IMB & & & \\
\hline$p \rightarrow \pi^{+} \bar{v}$ & $10^{(32-39)} \mathrm{y}$ & $2.5 \times 10^{31} y$ & Soudan & & & \\
\hline
\end{tabular}

${ }^{a}$ The modes shown represent a small sample of the more than 75 decay modes with limits on baryon lifetimes. The modes and current bound on lifetime for the modes are discussed in K. Nakamura, K. Hagiwara, K. Hikasa, et al. 2010. Review of particle physics, Journal of Physics G-Nuclear and Particle Physics 37: 075021.

${ }^{b}$ Predicted rates are estimates $(\mathrm{y}=$ years). Taken from P. Nath and P.F. Perez. 2007. Proton stability in grand unified theories, in strings and in branes, Physics Reports-Review Section of Physics Letters 441: 191.

'Super-K refers to the Super-Kamiokande experiment; previous experiments using a similar technique but smaller detectors were the IMB (Irvine-Michigan-Brookhaven) and Kamiokande experiments. A different technique, but with considerably less mass, was used by experiments at Soudan. (All experiments shown were performed underground.)

${ }^{d}$ Expected bound on lifetime by 2030, sensitivities for the first two decay modes expected by SuperKamiokande, and two possible detector configurations are shown in the rightmost three columns, as estimated in M. Bass, M. Bishai, E. Blaufuss, et al. 2011. "A study of the physics potential of the longbaseline neutrino experiment project with an extensive set of beam, near detector and far detector configurations," LBNE-PWG-002, INT-PUB-11-002, January. 
decay working group. ${ }^{15}$ The predicted rates are taken from a variety of popular unified models, such as SO(10) grand unification, split supersymmetry, models with extra dimensions at the GUT scale, etc. ${ }^{16}$ The wide range of predictions is indicative of the difficulties in obtaining definitive theoretical guidance, though the current bounds on some modes (particularly $\mathrm{K}^{+} \overline{\mathrm{v}}$ ) are already constraining model building.

Note that an order of magnitude improvement over the projected future SuperKamiokande bounds does not seem to be possible on a 10 -year timescale with the detectors being discussed for next-generation long-baseline neutrino experiments. Nonetheless, less ambitious improvement is possible, notably in the theoretically well-motivated $\mathrm{p} \rightarrow \mathrm{K}^{+}+\bar{v}$ mode. For these modes, a water Cherenkov detector would offer better statistics than the LAr option, but it would still have significant backgrounds and/or poor detection efficiency. LAr technology might provide much better control of backgrounds, offering improvement in this mode and possibly several other modes, particularly with final-state kaons. On the other hand, the LAr option does not offer improvement over Super-Kamiokande for the $\pi^{0} \mathrm{e}^{+}$mode, where the detection efficiency is high.

\section{Summary}

The search for proton decay is compelling science. There are theoretically persuasive reasons to expect that the proton will be found to be unstable, and proton decay provides a unique direct window into the physics of grand unification and the origin of matter. Decay rates and specific modes cannot be predicted given our current knowledge. Nonetheless, the range of lifetimes predicted by various theories has a large overlap with the sensitivity of current experiments, and current bounds have ruled out the simplest models and place severe constraints on others. The extension of experimental sensitivity provided by the large underground detector of a LBNE could credibly produce the important discovery of proton decay, although there are no guarantees. For this reason, the increased experimental reach is insufficient to be the primary factor in the choice of neutrino detector technology or detector siting. Each of the two detector technology options offers some, but different, promise for increased sensitivity to proton decay.

\section{Conclusion: The stability of the proton is a crucial, fundamental scientific question and can be studied by the large underground detector of a long-}

\footnotetext{
${ }^{15}$ M. Bass, M. Bishai, E. Blaufuss, et al. 2011. A study of the physics potential of the longbaseline neutrino experiment project with an extensive set of beam, near detector and far detector configurations, LBNE-PWG-002, INT-PUB-11-002, January. Available at http://www.int.washington. edu/PROGRAMS/10-2b/LBNEPhysicsReport.pdf. Last accessed on November 15, 2011.

${ }^{16}$ See, for example, P. Nath and P.F. Perez. 2007. Proton stability in grand unified theories, in strings and in branes, Physics Reports-Review Section of Physics Letters 441: 191.
} 


\begin{abstract}
baseline neutrino experiment. This capability would be of great scientific interest and would add significant value to the neutrino oscillation experiment. However, the sensitivity is not so important as to make the search for proton decay the primary consideration in choosing neutrino detector technology or a site for the experiment.
\end{abstract}

\title{
Nuclear Astrophysics
}

\section{Overview}

Understanding nuclear processes is critical to interpreting a number of astrophysical observations that range from stellar energy generation and the formation of solar neutrinos to elemental and isotopic abundances of the elements in the Universe. In particular, measurements of low-energy cross sections are key inputs to the nuclear reaction network calculations used to model these astrophysical phenomena. Thermonuclear reactions in stars occur in a narrow energy window-the so-called Gamow peak - with typical energies in the range of $\mathrm{keV}$ (for the Sun) to $\mathrm{MeV}$ (for explosive stellar processes). For a reaction to occur at these low energies, it is necessary for the participating nuclei to tunnel through the Coulomb barrier, which dramatically reduces the reaction cross section. While theoretical extrapolations can be used to extend higher-energy measurements into the verylow-energy astrophysical regime, such extrapolations have large uncertainties, in particular when low-energy resonances are present. Figure 3.6 shows a typical measurement, where the astrophysical S-factor is plotted versus the reaction centerof-mass energy. The S-factor is extracted from the cross section by dividing out the exponential suppression due to the Coulomb barrier.

Measuring nuclear cross sections at stellar energies requires high luminosities and low backgrounds. The counting rates of some of these stellar reactions can be as low as a few counts per day and the counts from these reactions cannot be reliably measured in the presence of cosmic ray background because of the low signal-tobackground ratio. This challenge led to the first underground accelerator facility at the Gran Sasso facility, the Laboratory for Underground Nuclear Astrophysics (LUNA). In this facility several key reactions were studied for the first time at the relevant solar energy. Following the success of the LUNA facility, a number of new initiatives to construct larger underground accelerator facilities were proposed because LUNA is somewhat limited in space. These proposals include designs for facilities in Germany, Spain, Romania, India, the United Kingdom, and the United States. The project proposed for Homestake is at the 4,400 m.w.e. level. Known as the Dakota Ion Accelerator for Nuclear Astrophysics (DIANA), it is one of the most ambitious. After addressing the general science case for a new underground accelerator facility, the report discusses the merits of a U.S. facility. 


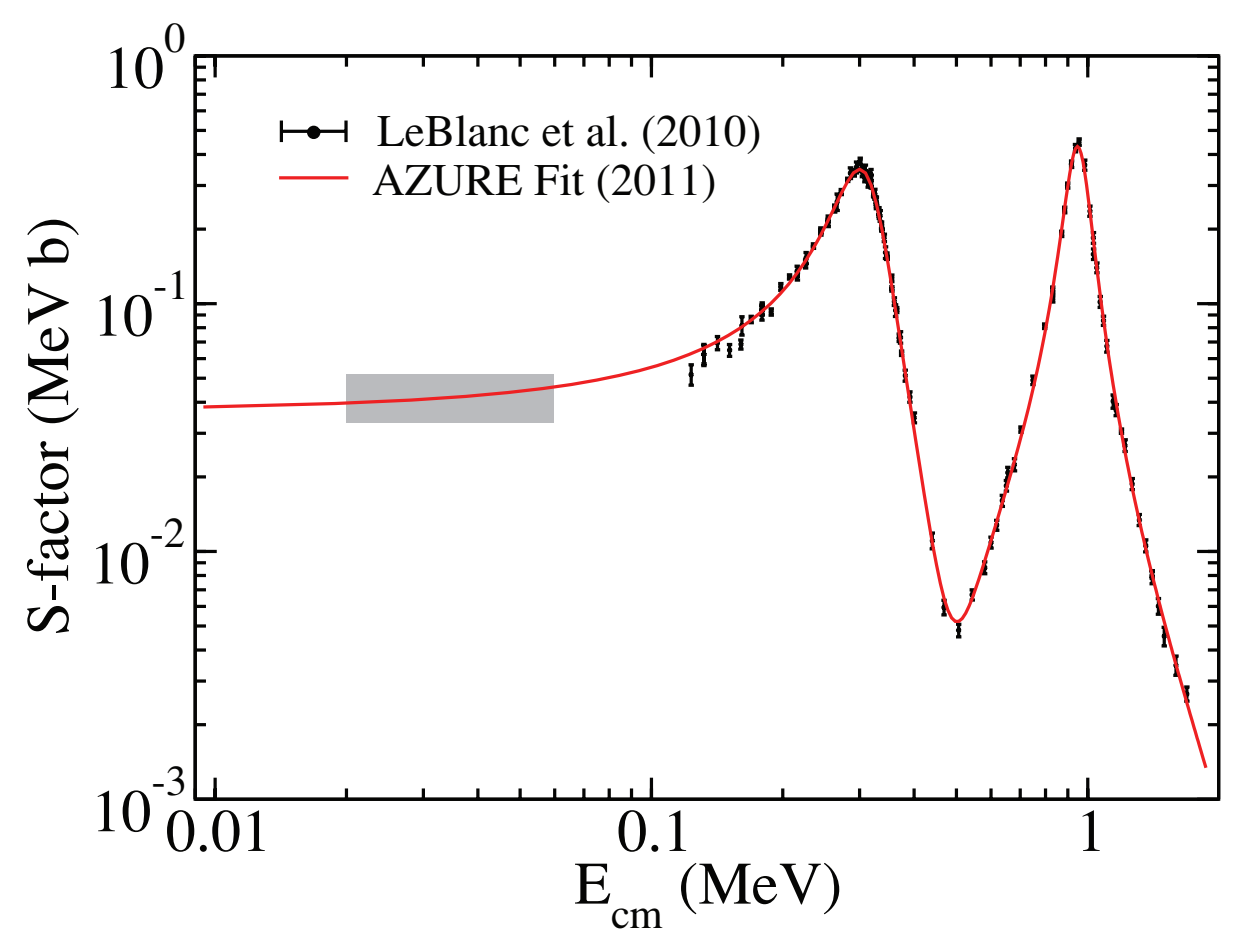

FIGURE 3.6 S-factor vs. center-of-mass energy for the ${ }^{15} \mathrm{~N}(\mathrm{p}, \gamma)^{16} 0$ reaction. The shaded box indicates the astrophysically important energy range-well below the measured data. SOURCE: Data from P.J. LeBlanc, G. Imbriani, J. Görres, et al. 2010. Physical Review C 82: 055804. Graph courtesy of Michael Wiescher, University of Notre Dame.

\section{Science Case}

DIANA involves the study of three important processes in nuclear astrophysics: (1) solar neutrino production, (2) nucleosynthesis in late-stage stellar burning as a precursor to white dwarf and supernova formation, and (3) the production of elements heavier than iron in neutron-rich nucleosynthesis. Each of these processes requires improved measurements of low-energy nuclear cross sections.

- Solar neutrinos flux was first studied as a means of exploring the thermal and compositional structure of the solar interior. This decades-long study helped transform our understanding of fundamental interactions by suggesting that neutrinos are massive and undergo flavor-changing oscillations. As more is learned about those parameters, it is important to return to solar neutrinos as a source of information about the Sun's processes. In particular, while the neutrinos from the proton-proton ( $p-p)$ chain (where protons are transformed into helium by sequential fusion) have been carefully studied, 
the flux of neutrinos from the carbon-nitrogen-oxygen (CNO) cycle is not well understood, primarily because of uncertainties in the nuclear processes that produce neutrinos. Improved measurements of relevant reactions, such as ${ }^{14} \mathrm{~N}(\mathrm{p}, \gamma){ }^{15} \mathrm{O}$ and ${ }^{15} \mathrm{~N}(\mathrm{p}, \gamma){ }^{16} \mathrm{O}$, would allow us to use the $\mathrm{CNO}$ neutrinos as a probe of the so-called metallicity of the solar interior. Metallicity is the abundance of elements heavier than helium in the initial solar core. The abundance of these elements indicates the extent of the nuclear processing that occurred in the material that formed our solar system.

- The LUNA program has focused on reactions relevant to hydrogen burning, the main energy generation process in the Sun. However, red giant stars and asymptotic giant branch (AGB) stars are fueled mainly by helium burning. Helium burning begins at elevated temperatures with the triple-alpha process that allows three helium nuclei to fuse into ${ }^{12} \mathrm{C}$. Radiative capture of alpha particles on ${ }^{12} \mathrm{C}$ to produce ${ }^{16} \mathrm{O}$ and the subsequent alpha capture on ${ }^{16} \mathrm{O}$ set the stage for carbon burning, a series of fusion reactions between carbon nuclei and carbon and oxygen nuclei. These burning processes then greatly influence the light-element composition of the star. This composition is a key component in the calculation of nova and supernova ignition. These radiative capture reactions and fusion reactions are poorly measured, especially near the relevant stellar energies at and below $1 \mathrm{MeV}$.

- The slow neutron capture process or s-process is thought to be the source of a large number of elements heavier than iron. During the later stages of helium burning, alpha particle capture on certain isotopes of carbon, oxygen, and neon- ${ }^{13} \mathrm{C}(\alpha, \mathrm{n}){ }^{16} \mathrm{O},{ }^{17} \mathrm{O}(\alpha, \mathrm{n}){ }^{20} \mathrm{Ne}$, and ${ }^{22} \mathrm{Ne}(\alpha, \mathrm{n}){ }^{25} \mathrm{Mg}$ - can produce copious amounts of neutrons whose sequential capture on seed nuclei produces the heavier elements.

These three science topics are among the most compelling in the field of nuclear astrophysics, which itself was noted as being one of three key intellectual directions for nuclear physics in the 2007 long-range plan for nuclear physics. ${ }^{17}$ The main thrusts within nuclear astrophysics are exploring the structure of nuclei far from stability, understanding the nuclear equation of state, and measurements of low-energy nuclear cross sections. The first two thrusts are key elements of the new facility for rare isotope beams (FRIB) accelerator to be constructed at Michigan State University. The third thrust, the understanding of low-energy nuclear reactions, is the key focus of DIANA, which could effectively complement the nuclear astrophysics science to be addressed by the FRIB accelerator.

\footnotetext{
${ }^{17}$ DOE/NSF. 2007. The Frontiers of Nuclear Science: A Long Range Plan. Report of the Nuclear Science Advisory Committee.
} 


\section{Experimental Aspects}

Measurements of all of the low-energy cross sections discussed above are hampered by the ultra-low event rates at the relevant stellar energies and by background contamination. The pioneering LUNA facility at Gran Sasso has already demonstrated that with sufficient suppression of cosmic ray background, cross section measurements can be performed at much lower energies than is possible aboveground.

The proposed DIANA facility consists of two high-current accelerators (about 100 times the luminosity of LUNA), whose beams can be directed to a number of target stations. The lower energy accelerator covers the energy range 50-400 keV, while the higher energy accelerator extends from $400 \mathrm{keV}$ (to match the lower energy machine) to $3 \mathrm{MeV}$ for singly charged ions. Beams from both accelerators can be directed to the same high-density gas target stations in order to map out key reactions over a larger energy range, thus allowing the study of a variety of burning processes in stars. A concept model for the accelerator complex is shown in Figure 3.7. This complex requires a cavern approximately $20 \mathrm{~m}$ high by $20 \mathrm{~m}$ wide by $45 \mathrm{~m}$ long.

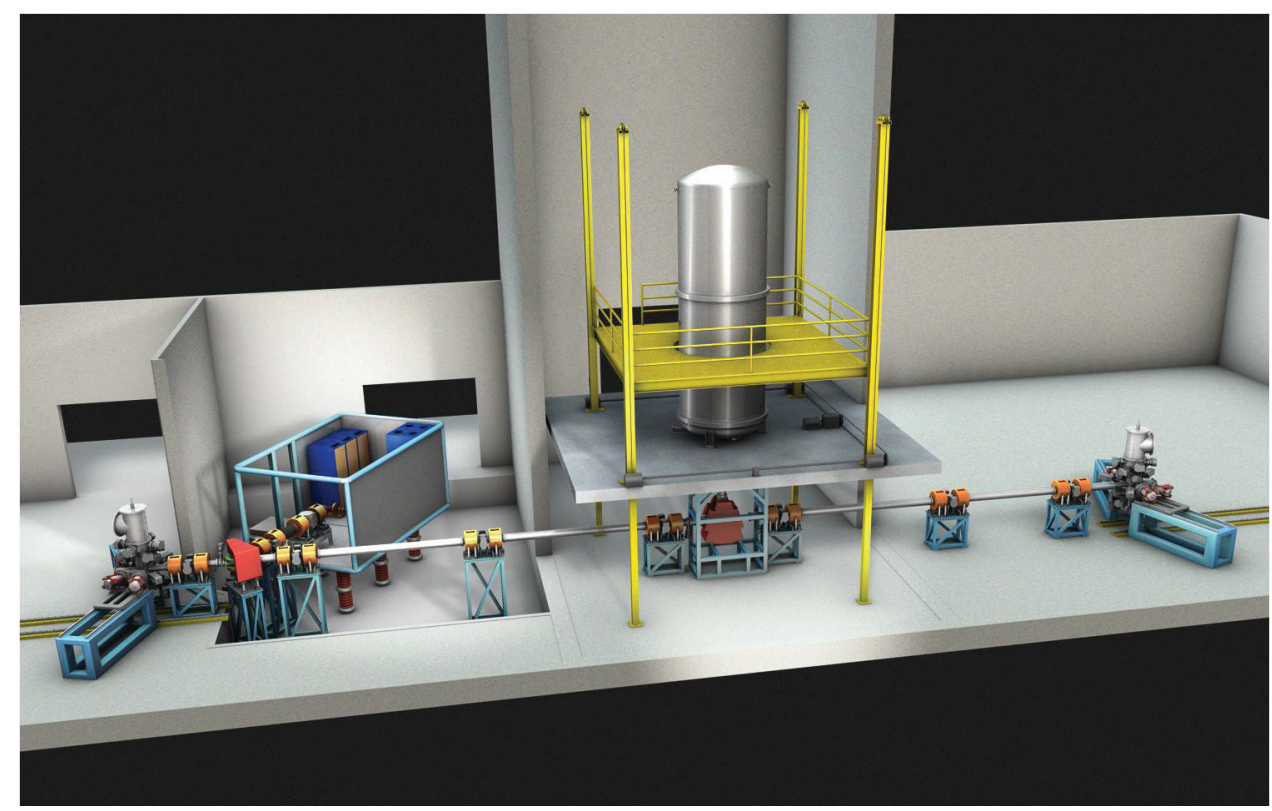

FIGURE 3.7 Concept model for the DIANA accelerator facility. The lower energy machine is shown on the left, and the higher energy machine, mounted vertically, is in the center. Two target stations are shown at the far left and far right. SOURCE: Michael Wiescher, University of Notre Dame, presentation to the committee on December 14, 2010. Image courtesy of Daniela Leitner, co-principal investigator of the DIANA project. 
A key aspect of the DIANA facility is the design of the target stations. These must be able to handle the very high beam currents while keeping beam-induced backgrounds to a minimum. High-density gas target systems will be used in combination with state-of-the-art gamma-ray and neutron detectors. Figure 3.8 demonstrates the improvement in background levels (on a logarithmic scale) for gamma-ray detection that can be achieved under various shielding configurations. The principal experimental advantages of DIANA over existing experiments such as LUNA are advances in design that allow it to significantly reduce background counts, as shown in Figure 3.8.

Clearly, a significant portion of the international community is interested in the science of low-energy nuclear astrophysics. As discussed above, in addition to the LUNA facility at Gran Sasso (3,500 m.w.e.) and the proposed DIANA facility, other underground low-energy accelerator facilities are being considered: Dresden (Germany, 110 m.w.e.), Canfranc (Spain, 2,500 m.w.e.), Praid (Romania, 900 m.w.e.), Boulby (U.K., 2,800 m.w.e.), and INO (India, 3,500 m.w.e.). The DIANA project itself has a number of international partners.

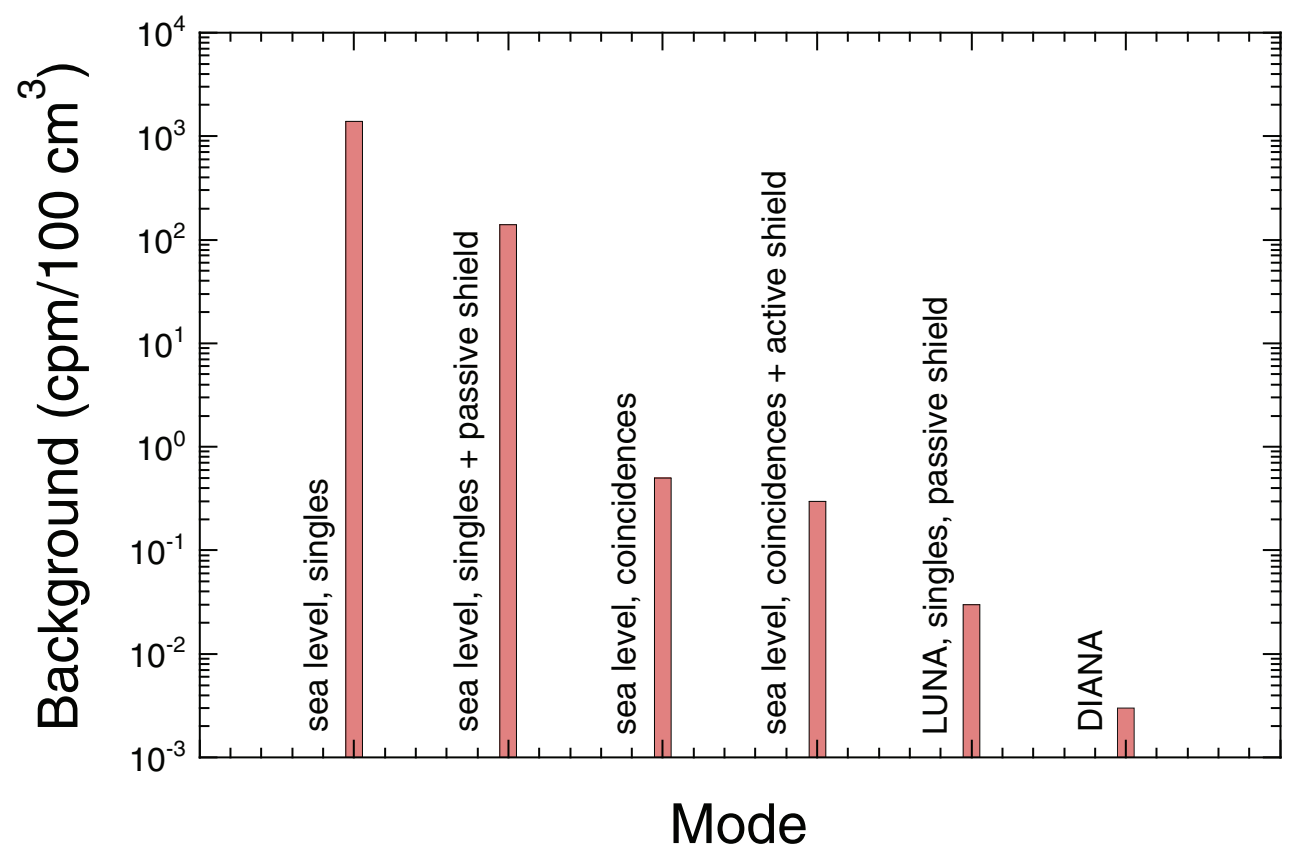

FIGURE 3.8 Gamma-ray background levels for different shielding configurations. With advanced detector designs and a depth of approximately 4,000 m.w.e., DIANA could achieve a background one-tenth that of LUNA. SOURCE: Original figure courtesy of Christian lliadis, University of North Carolina at Chapel Hill; adapted to include DIANA data. 
The design for the DIANA facility is fairly well advanced, and construction could begin in the next several years. Should a new underground laboratory in the United States not be pursued, the collaboration has also begun investigating opportunities at the WIPP facility (1,600 m.w.e.) in New Mexico. The technical feasibility of the DIANA underground facility does not appear to pose a major risk for the project. The accelerators are largely based on existing systems that have been successfully implemented. Some research and development will be required to develop target stations that can handle the high currents from the accelerators. Most of this work can be performed aboveground and is already under way at the University of Notre Dame and the University of North Carolina. New detection techniques will take advantage of the low background environment.

A potential complication associated with an underground accelerator facility operating in conjunction with other very-low-background experiments is reduced sensitivity in those other experiments due to accelerator-related background. However, straightforward means can be implemented to eliminate interference with other experiments. Thus a well-isolated cavern is required with adequate shielding to reduce background to manageable levels. In addition, for experiments that require higher energies, which are more likely to produce elevated background levels, the accelerator can be operated in a low-duty-cycle pulsed mode. In this mode, the timing of the accelerator can be included in the data acquisition systems of the other experiments to allow for studies of possibly elevated backgrounds or vetoing of signals during the beam pulses. However, Monte Carlo simulations performed by the collaboration indicate that no additional neutron flux will be present outside the DIANA cavity. Careful communication between experiments will be essential to ensure that periods of potentially high background runs have minimal impact on other experiments. These measures for preventing interference with other experiments are not expected to be costly.

\section{Summary}

The importance of the science questions that DIANA could address makes this an exciting research opportunity for nuclear physics. The ultralow backgrounds at such an underground facility will enable precise measurements of very-low-yield stellar reaction rates that are key to elucidating important astrophysics processes. The LUNA facility at Gran Sasso has already demonstrated the usefulness of an underground accelerator for understanding the hydrogen burning process. A more advanced facility such as DIANA will shed light on other key burning processes in stars and on the production of elements heavier than iron.

Conclusion: A small underground accelerator to enable measurements of low-energy nuclear cross sections would be scientifically important. These 


\title{
measurements are needed to elucidate fundamental astrophysical processes such as thermonuclear reactions and the production of heavy elements in the Sun and the stars.
}

\author{
Neutrino Astrophysics
}

\section{Overview}

The possibility of using neutrinos to make unique and valuable contributions to astronomy and astrophysics has been recognized since neutrinos were discovered. Because neutrinos interact only weakly with matter allows them to be used as probes of processes that occur in dense regions from which photons cannot escape. In addition, because neutrinos play a central role in the dynamics of a number of important astronomical systems such as supernovas and solar cores, our understanding of these systems cannot be complete until the emitted fluxes of neutrinos can be accurately measured. Similarly, it has long been realized that the properties of neutrinos can be uniquely tested using astrophysical systems as neutrino sources and the Universe itself as our laboratory.

To date, the Sun and supernova SN1987A are the only sources that have provided a detected neutrino signal. However, the present and upcoming generation of large underground detectors hopefully will increase our neutrino source catalog to include a galactic supernova, the integrated flux of all supernovas throughout the history of the Universe, ultra-high-energy sources such as active galactic nuclei and gamma-ray bursts, and even Earth itself.

\section{Scientific Landscape-Neutrino Astrophysics}

Supernovas are spectacular stellar explosions in which the energy released in a few weeks is comparable to that expended by the Sun during its entire lifetime. Further, supernovas are believed to play a crucial role in the history of the Universe. For example, the heavy elements in cosmic rays are synthesized in massive stars and ejected in supernova explosions. So, it is not an exaggeration to say that life itself would not have been possible without supernovas. It also appears that supernovas play essential roles in galaxy formation and in reenergizing the process of star formation at later times in the life of a galaxy. From examining the decay products of radioactive isotopes, it appears that a nearby supernova seeded the elemental composition of our solar system and may have contributed to its creation.

In spite of their importance, supernovas are not yet well understood. Although baseline models exist, there are still many uncertainties, and significant problems stem from trying to determine the fundamental processes occurring in the center of the explosion from the relatively late-time optical light curve. Core-collapse 
(Type II) supernovas are those in which a massive star exhausts its nuclear fuel; heavier and heavier elements are exhausted until a nickel-iron core forms that can no longer support the weight of the star. In the resulting explosion, more than 99 percent of the energy released comes out in the form of neutrinos that are largely emitted in the first 10-20 s. The bulk of the neutrinos are emitted at energies below $40 \mathrm{MeV}$, and it is expected that the emitted neutrinos are roughly evenly distributed among the three flavors and particles and antiparticles. However, to estimate the expected neutrino signal at Earth from a galactic supernova, flavor oscillations must be taken into account and, in fact, the detected neutrino signal can provide crucial information about not only supernovas but also the neutrino.

The detection of 19 neutrinos from SN1987A in the Large Magellanic Cloud by the first generation of underground water Cherenkov detectors at Kamiokande in Japan and the onetime Irvine-Michigan-Brookhaven detector was a historic event that demonstrated the possibility of supernova neutrino astronomy. The detection of a core-collapse supernova in our galaxy by a large LBNE detector would, in turn, provide a wealth of scientific information, relating to our understanding of particle physics as well as our fundamental picture of supernovas.

Supernovas in our galaxy are relatively rare occurrences. In fact, the last recorded event occurred more than 300 years ago. However, we have good evidence that numerous supernovas occurred in our galaxy since then but were obscured from our view. From a variety of inferences, the core-collapse supernova rate in the Milky Way is estimated to be approximately two per century, which means that a detector would need to operate for more than 20 years to have a significant chance of catching an event. Because these events are so rare, it is essential that multiple detectors are available worldwide, not only to ensure that at least one detector is operational when the neutrinos reach Earth but also to maximize the scientific output should more than one detector see the same event.

\section{Experimental Details}

Although moderately sized detectors built to study solar neutrinos (or to search for dark matter or neutrinoless double-beta decay) could, in some cases, detect neutrinos from a nearby supernova, it is the large detectors proposed for LBNE that would greatly increase our capabilities. A water Cherenkov detector of $300 \mathrm{kTon}$ scale would detect a very large number, estimated to be on the order of 20,000, of events from a supernova in the galactic center (i.e., at a distance of 8.5 kiloparsecs). Most of the recorded events would be antielectron neutrinos, detected via the inverse-beta-decay (IBD) reaction, with different models varying by factors of three to four in their prediction of the expected neutrino event rate. A statistically significant number of neutrinos would also be detected through elastic-scattering (ES) and charged-current (CC) interactions, with the latter events 
providing directional information. Thus, the water Cherenkov detector of LBNE would clearly distinguish between various models that describe core collapse, and the relative numbers of neutrinos detected via the IBD, ES, and CC interactions would help us to know the flavor composition of the flux, which could be further improved by the addition of Gd to the water to permit neutron tagging.

A LAr detector of the size envisioned for LBNE would detect on the order of 1,000 neutrino events from a galactic core-collapse supernova, where most of the signal would be in the form of electron neutrinos detected via the CC interaction, $v_{\mathrm{e}}+{ }^{40} \mathrm{Ar} \rightarrow \mathrm{e}^{-}+{ }^{40} \mathrm{~K}^{*}$. The reduction by more than an order of magnitude in the neutrino signal in the LAr detector relative to the water Cherenkov detector is due partly to its smaller size and partly to the relevant interaction cross sections. However, the improved energy resolution of liquid argon could partly compensate for the lower statistics. For example, the expected sensitivity in the ability to differentiate between the normal and inverted neutrino mass hierarchies is comparable for the two detectors. It is important to note that the backgrounds in a large LAr detector at these low energies (threshold energy $\sim 2 \mathrm{MeV}$ ) are not yet well known and could be significant at the shallower depths of 300 to $600 \mathrm{ft}$ being considered for that detector.

Detecting neutrinos from supernovas outside our galaxy is largely a question of probability and distance, since the flux will vary as $1 / \mathrm{r}^{2}$, where $\mathrm{r}$ is the distance between the supernova and Earth. Thus, events occurring within the satellite system of the Milky Way would certainly be detectable, but events occurring further out in the local group (e.g., Andromeda) would not. However, an important potential exists - namely, the detection of the diffuse neutrino signal arising from all supernovas that have occurred during the lifetime of the Universe. Detection of these so-called supernova relic neutrinos would be an experimental tour de force. The flux and spectrum of the relic neutrinos could tell us about the uniformity of the supernova neutrino signal, whether SN1987A was a representative explosion, and whether there exists a component of supernovas that does not shine brightly in the optical band. Importantly, the diffuse neutrino flux predicted by different models is uncertain by a factor of approximately 12 .

The spectrum of the relic neutrinos will have the same shape as the neutrinos from individual supernovas, but the signal will lack a distinct temporal signature since the relic neutrino flux is steady-state. Consequently, there exists only a small window of neutrino energy, between 20 and $30 \mathrm{MeV}$, where the relic neutrinos may be detectable above background. Below this window, solar neutrinos swamp the signal, and above it, atmospheric neutrinos dominate. No evidence for a signal of relic neutrinos was seen in a long exposure at Super-Kamiokande (approximately 1,500 days of SK-I and 800 days of SK-II), and the upper limit on the flux of supernova relic neutrinos from these data is just reaching the largest theoretically predicted flux. A large water Cherenkov detector similar to that proposed for LBNE would 
substantially improve on this limit and could well detect a statistically significant signal. The ability to confidently see a signal with a water Cherenkov detector in the baseline configuration (15 percent photocathode coverage, no Gd doping) would be marginal, given the large uncertainty in the flux level. However a detector in the enhanced configuration (30 percent photocathode coverage, Gd doping) would cover most of the parameter space and could confidently expect to see a signal. The LAr detector option for LBNE is too small to significantly improve on the capabilities of Super-Kamiokande.

\section{Summary}

Neutrinos are expected to be produced in most astrophysical sources, and their detection on Earth could lead to profound insights about the relevant astrophysics in the sources themselves, as well as an important understanding of the properties of the neutrino. Indeed, the detection of neutrinos from the Sun and SN1987A were crucial advances in the development of neutrino astrophysics. A large underground detector for long-baseline neutrinos would serve as an excellent detector of neutrinos from a nearby supernova, and it might also be possible to see the first evidence of the relic neutrinos from supernovas that have occurred over the history of the Universe. Although the rate of supernovas in our galaxy is relatively small, there is a reasonable possibility of one happening within a 20 -year period. A large underground detector like the one envisioned for DUSEL would detect a large number of neutrinos from a galactic supernova. This would greatly advance our understanding of these important sources and could shed new light on the makeup of the neutrino itself.

Conclusion: Neutrinos from supernovas can be studied by a large underground detector of a long-baseline neutrino experiment, making a unique and valuable contribution to our understanding of one of the most important astrophysical phenomena. This capability of the neutrino oscillation experiment would be of great scientific interest and add a significant value to that experiment. However, the sensitivity for detecting neutrinos from supernovas is not so important as to make it the primary consideration in choosing neutrino detector technology or a site for the experiment.

\section{NONPHYSICS SCIENCE AND ENGINEERING PROGRAMS}

\section{Overview}

While the principal focus of the DUSEL program is the pursuit of physics research, the development of such a facility would provide rich research opportunities 
for other fields. The environments that exist in underground facilities at depths of a few hundred to several thousand meters or more are complex and offer systems with strongly coupled thermal, hydrological, mechanical, chemical, and biological characteristics. The nature of such an environment, including how its components engage and influence one another, is the focus of both applied and basic research in fields that range from engineering applications to geological studies of geomechanics and geophysics, and to research into biological systems in extreme environments. Much of this research can be carried out only in situ, since many of the important events occur on time and spatial scales that cannot be replicated through sampling and intermittent tests.

Subsurface engineering research includes work related to fairly traditional extractive activities that arise in petroleum drilling and mining and civil engineering issues associated with rock slopes, dam foundations, tunnels, rapid transit, and subsurface city infrastructure. However, those more traditional research fields are now joined by research in areas such as "enhanced" geothermal systems, unconventional sources of natural gas, and an ever-widening variety of applications of the subsurface for isolating materials such as nuclear waste and $\mathrm{CO}_{2}$.

For the geo- and biosciences, the nature of the environment itself and how it responds to disturbances offers a wide range of research opportunities. Many rock types have exceedingly low porosity and permeability, and at great depth, fractures ${ }^{18}$ are commonly the main conduits for fluid flow, the main determinant of rock strength, and the locus of seismicity. Although these fractures are critical to many aspects of rock behavior and are present over a wide range of scales they are exceedingly poorly understood for the simple reason that they are easily missed by conventional subsurface probes such as well bores. A subsurface environment at great depth may also enable the existence of microbiological communities. How those communities arise and survive is the result of a complex engagement among the chemical, hydrological, and thermal characteristics of the underground environment.

In this section of the report, the committee discusses the experiments that have been proposed for incorporation into the initial suite of research to take place at

\footnotetext{
${ }^{18}$ A "fracture" is a break in a rock caused by brittle failure. A "fault" is a fracture whose opposite sides have been offset parallel to the fracture surface (i.e., shearing offset). A fault is a fracture, but not every fracture is a fault. In the literature and in casual conversation "fracture" frequently refers to fractures that display no fracture-parallel offset-that is, those that are not faults. It would be better to call these features "opening-mode fractures" (or extension fractures or cracks or joints). The only displacement accommodated by these fractures is parting of opposing walls (widening of the aperture).
} 
DUSEL, ${ }^{19}$ but possible future experiments are many and are briefly touched upon at the end of this section.

\section{Subsurface Engineering Challenges}

Rock at depth has mechanical characteristics that set it apart from other materials. Rock is preloaded by vertical (gravitational) and lateral (tectonic) forces. It is common for rock to be extensively fractured and folded owing to deformation processes occurring over many millions of years. The rock is a combination of a solid "skeletal" (matrix) component and a system of interconnected fluid-filled pores within the skeleton. The forces are transmitted in part by the solid rock, which sustains different forces (or stresses) in different directions, and in part by the fluids, which develop a pressure in the pores. It is a dynamic system with the tectonic forces increasing continuously, albeit at a very slow rate, until some part of the system-commonly a fracture or a fault-is overloaded and slip occurs, until the system reaches a new equilibrium. Depending on the force-deformation characteristics of the fault and those of the surrounding rock mass, this slip may occur violently, producing an earthquake and seismic waves, or slowly, as a gradual process.

Figure 3.9 illustrates some of these factors. When subjected to a change in load, the rock deforms (as indicated in the lower diagram), eventually reaching a limit when the internal structure starts to disintegrate. In practice, these load changes are usually a result of disturbing the preexisting equilibrium by, for example, the introduction of an excavation (borehole, tunnel, or mine) or the injection or removal of fluid. An excavation changes loads on rock in its vicinity, as does localized fluid, for which the excavation serves as a "sink." The intensity of the load decreases with distance from the "disturbance." However, elastic energy in the outer region is available to "feed" the disintegration. Depending on the type of rock and its unloading characteristics, the disintegration is sometimes violent, as in the case of earthquakes. The deformation characteristics of the rock will change as the number of fractures increases (Figure 3.9, upper left diagram), and as the duration (Figure 3.9, upper right) of the load increases. ${ }^{20}$

\footnotetext{
${ }^{19} \mathrm{~A}$ science review of six of the proposed geosciences/engineering experiments was recently conducted by a subcommittee of the National Science Foundation's (NSF's) Deep Underground Science and Engineering Laboratory (NSF. 2011. AC-GEO Subcommittee, Science Review Panel Report, April 1).

${ }^{20}$ The diagram indicates that the rock reaches a peak load beyond which it disintegrates progressively. This is the case when the lateral confining pressure is reduced (e.g., by the excavation process). In the interior of a rock mass, the rock remains "elastic" but may still undergo long-term strength changes owing to the thermochemical effects of the fluids circulating through the rock. As the depth and temperature increase, the rock will change progressively from a brittle to a ductile material.
} 
“We don't know the rock mass strength. That is why we need an international society” $\quad$ Muller, May 24, 1962

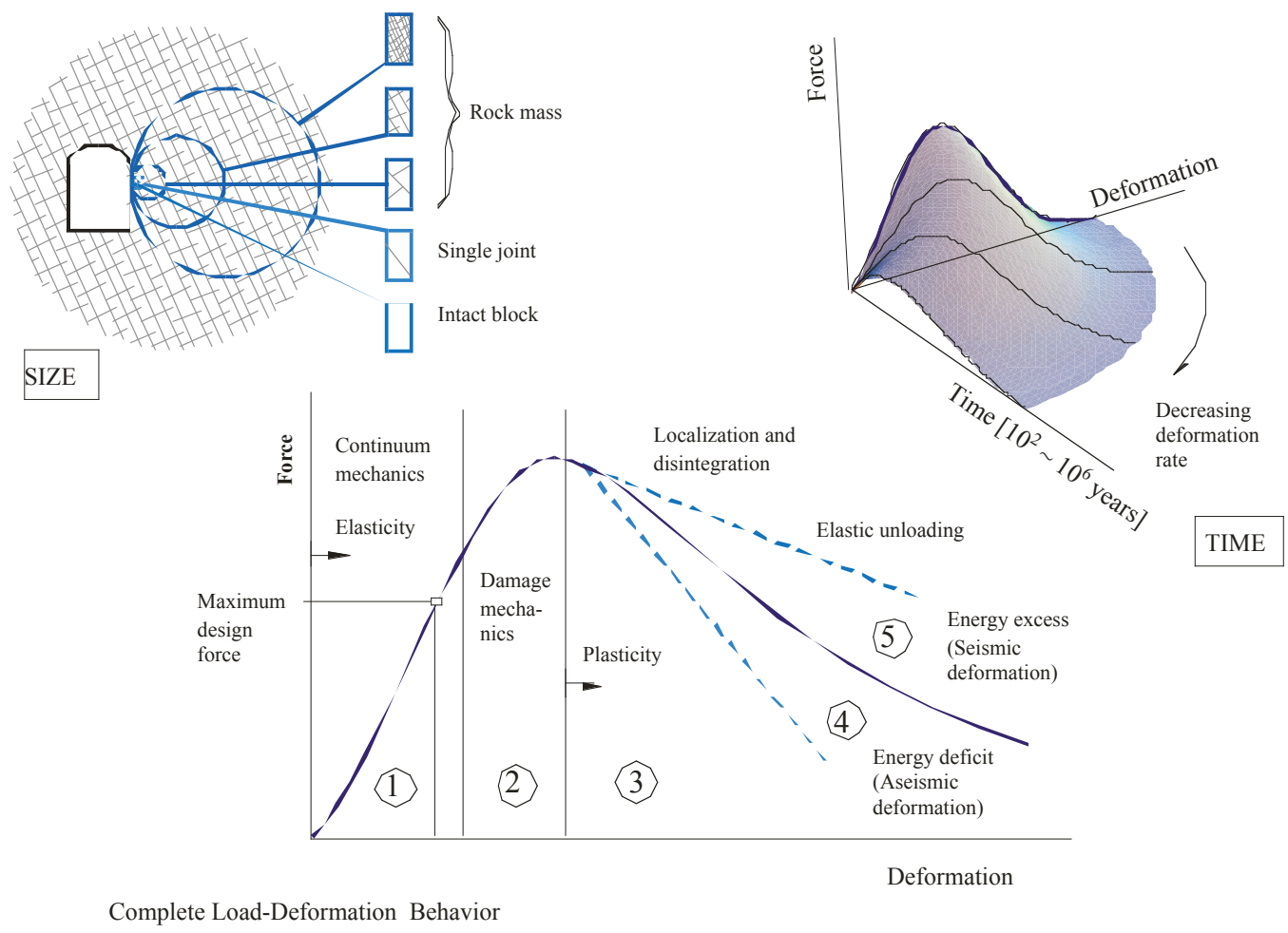

FIGURE 3.9 Effects of size and timescales on the mechanical response of rock to applied loads. The quotation, from a pioneer and professor of subsurface engineering, Leopold Müller, referred to the central challenge of rock mechanics. Numerical models now allow rock mass strength to be predicted. In situ tests such as those proposed for DUSEL will allow these predictions to be evaluated and will be a major step forward in a 50-year-long quest. SOURCE: Courtesy of Charles Fairhurst, Itasca Consulting Group, Inc.; from Fairhurst Müller Lecture, 2003. 10th International Society for Rock Mechanics Congress, Johannesburg.

Engineering projects can be kilometers in linear extent. In some cases, such as nuclear waste isolation and carbon sequestration, the performance of the engineered system must be assessed over very long periods (tens to thousands of years or longer). Projects in petroleum engineering now extend to depths on the order of $10 \mathrm{~km}$, where rock temperatures may exceed $350^{\circ} \mathrm{C}$ and in situ rock stresses are on the order of $250 \mathrm{MPa}$. Proposed geothermal energy projects involve comparable depths. Part of the rock pressure is supported by fluids circulating through connected pores in the rock. Chemical reactions between the fluids and the rock are complex and not well understood but very important in designing effective longterm heat-exchange systems for geothermal energy production. 
The advent of high-capacity computers now allows large-scale features to be modeled and deformation behavior predicted. However, testing the validity of computer predictions requires experiments to be conducted in situ. Attempts have been made to obtain some insights into in situ behavior through the study of rock exposed during events such as mining operations and the construction of dam foundations. However, because the primary purpose of these excavations is not research, these sites are far from optimal and typically do not allow for the careful design and instrumentation of experiments or for the conduct of experiments over extended periods of time. Having extensive underground space available for regulated long-term experiments, such as those proposed for DUSEL, would help to address these shortfalls.

\section{Geoscience Challenges}

It may seem surprising that basic questions of subsurface geomechanics, geohydrology, geochemistry, and geophysics remain given that subsurface studies have been central to geology since its inception. Using coring techniques, geoscientists acquire samples $^{21}$ from great depths, and they regularly deploy sophisticated geophysical well-logging tools to document a wide range of rock and fluid properties at depth. These measurements are augmented by powerful geophysical seismic methods for imaging undrilled areas. Despite the inherent inaccessibility and complexity of subsurface environments, such tools are adequate for characterizing many rock properties. Thanks to the advent of geophysical logging tools that image or detect fractures at or near the well bore and coring procedures that succeed in fractured rock, log- and core-based methods usually provide some information on fracture attributes. For example, well bores that target large faults can provide rock samples to support physical and chemical investigations of earthquake zones. ${ }^{22}$

Nevertheless, for assessing other important attributes of rocks in these settings - fractures and faults and their relations to fluids, in situ stress, chemical reactions and microbiology — wellbore-based studies have important limitations. Data are commonly incomplete because meaningful samples of subsurface fracture networks are inherently difficult if not impossible to obtain. The actual process of core drilling into the rock produces stress changes that may change the core and render it unrepresentative of the rock from which it was sampled.

Fractures are commonly too small and widely spaced to be effectively sampled by well bores, ${ }^{23}$ and, owing to their small size, opening-mode fractures and many

\footnotetext{
${ }^{21}$ Typical cores are cylinders $\sim 10 \mathrm{~cm}$ or less in diameter and of arbitrary length but usually $<100 \mathrm{~m}$.

${ }^{22}$ For example the deep drilling component of EarthScope in the San Andreas fault near Parkfield, California. See http://www.earthscope.org/observatories/safod. Last accessed on September 26, 2011.

${ }^{23}$ W. Narr, D. Schechter, and L.B. Thompson. 2006. Naturally Fractured Reservoir Characterization. Richardson, Tex.: Society of Petroleum Engineers.
} 
faults are invisible to indirect geophysical investigation. ${ }^{24}$ The widespread distribution of fracture arrays and the small size of individual fractures mean that vital characteristics $^{25}$ of most subsurface fractures are little known. Well bores that do intersect fractures or faults may not be optimally located within the structure to provide insight into important processes. Because the geoscience data needed for breakthrough insights is inherently three-dimensional over a wide range of scales, small samples at a single point are bound to be inadequate, and they may provide no meaningful data or even misleading data.

Operating mines provide access to the underground but do not usually allow for long-term studies, impeding our understanding of fluid flow and its associated physical and chemical processes. An alternative to subsurface studies is the investigation of rocks that have been buried and then uplifted to the surface. These rocks may preserve evidence of faults and fracture arrays and the by-products of chemical reactions that existed at depth, but key features may be obscured or overprinted during uplift. Moreover, these fossilized records lack the essential dynamic context of tectonic, burial, and thermal loading, fluid flow, and chemical reactions.

Faults are important features that cross a wide spectrum of the geosciences and have important societal impacts beyond earthquakes. Understanding the nucleation and rupture of earthquakes on faults is a central theme of seismology and rock mechanics, and unraveling the history of slip is a central research area for structural geologists. The dynamic aspects of rock at depth have profound implications for engineering operations that perturb the subsurface, including drilling, hydraulic fracturing, and fluid storage. Further, mass transport and mineral deposition along faults is an important source of metal ores, and understanding these processes is a significant challenge to geochemists and economic geologists. Faults affect preferential pathways for fluids at a wide range of lengths and timescales. An important fraction of Earth's heat flow is carried by hydrothermal circulation through faults, and the circulation of cooler water through faults is a hydrogeologic process. Faults can also be a locus for microbial life.

Despite their significance, the study of stresses and strain deep in Earth's subsurface and their interaction with preexisting or growing fractures; moving or static fluids; and chemical or biochemical reactions is necessarily restricted to sparse point measurements in deep boreholes and deep mines ${ }^{26}$ that rarely include measurements over time ${ }^{27}$ and are seldom located in the most informative places

\footnotetext{
${ }^{24}$ The Leading Edge, v. 26, no. 9, September 2007.

${ }^{25}$ Such as length, height, and aperture distributions, connectivity, orientations, and patterns of mineral deposits, and variation of these attributes with position and rock type.

${ }^{26}$ T. Engelder. 1993. Stress Regimes in the Lithosphere. Princeton, N.J.: Princeton University Press, at 451 .

${ }^{27}$ NRC. 1996. Rock Fractures and Fluid Flow: Contemporary Understanding and Applications. Washington, D.C.: National Academy Press.
} 
or collected at the most interesting times. An example of a potentially interesting data set that is lacking and that illustrates the last two points would be measurements of all key parameters near the nucleation zone of an earthquake prior to, during, and after the event. The most desirable subsurface experimental setting would therefore enable observations over large volumes (hundreds of cubic meters to cubic kilometers) and for long periods of time (years to decades), providing researchers with the opportunity to target and perhaps even deliberately perturb ${ }^{28}$ specific key, instrumented areas within a given volume. Such a setting would allow systematic investigations of important interactions and the feedback on them that are suspected to exist among loading, fracture growth, closure or sealing, altered permeability and porosity and structure of the rock and fractures, altered composition of fluids, altered stress, and pressures, directions, and rates of fluid movement. For example, fluid pressure changes can alter a rock's elastic response to deforming forces, which could influence earthquake frequency and magnitude. As with permeability, variation in rock strain and stress as a function of measurement scale and sample position and size is not well understood because sufficiently large volumes of rock at depth have not been adequately measured or characterized.

Many fracture and fault attributes and their behavior with respect to processes covered by the disciplines of geomechanics, geohydrology, geochemistry, and geophysics could be addressed effectively in an underground laboratory. Such facilities would permit measurement of rock structure, fracture attributes, and their variability with size, depth, and distance across the excavation. The scale of observation has to be large enough to allow for the collection of meaningful evidence for coupled mechanical, geochemical, and microbiological processes occurring within the subsurface environment. These processes can play a vital role in how effectively fluids are stored in or transmitted through rock and how faults and opening-mode fractures behave over time spans of hours to decades to millennia and, thus, how they may respond to human intervention. The ability to investigate the rock volume after tracer tests or imaging may lead to improved techniques that can be applied elsewhere.

Access to the large rock volume would permit testing the hypothesis that Earth's crust is critically stressed and that some part of Earth is always close to failure by fracture. Significant rock permeability at depth may occur along critically stressed fractures. Mapping fractures, stress, and fluid flow within the subsurface will help geoscientists to confirm or extend theories about the mechanics of Earth deformation.

Any disturbance of the subsurface, be it "natural" - for instance by volcanic or seismic activity_or as a result of engineering, will change the preexisting

\footnotetext{
${ }^{28}$ Active experiments, such as placing heaters in the rock mass, might improve our understanding of how coupled mechanical, chemical, and fluid-flow behavior responds to environmental changes.
} 
equilibrium, sometimes dramatically, as in the case of surface tremors induced by fluid injection at depth.

The process of coring to obtain rock specimens from these subsurface environments can change their properties to an unknown extent. In some cases, the behavior of cores, the primary basis for much of university laboratory rock mechanics research to date, may not be representative of rock's behavior in situ.

\section{Bioscience Challenges}

Microorganisms have inhabited Earth for 3.5 billion years and hence have had a much longer time for adapting to life in a mineral world than some more recent microorganisms have had to adapt to life with higher organisms. During that long time some evolved mechanisms to capture energy from virtually every energy-yielding chemical redox couple. The more common inorganic reductants supporting microbial growth are $\mathrm{Fe}(\mathrm{II}), \mathrm{S}^{-2}, \mathrm{H}_{2}$, and $\mathrm{NH}_{4}^{+}$, while $\mathrm{Fe}(\mathrm{III}), \mathrm{NO}_{3}{ }^{-}$, $\mathrm{SO}_{4}^{-2}$ as well as $\mathrm{O}_{2}$ are common oxidants. Other minerals that are involved include but are not limited to Se, As, P, Mn, Cr, Co, U, and Zn. These minerals can also serve as electron donors and/or acceptors, supporting some microbial growth. Also, because of their long history, microbes are widely dispersed and serve as inocula in fissures within rocky materials, becoming available as life-sustaining niches. Besides their diversity in capturing energy, these microbes have also evolved adaptations to extreme conditions, such as long-term starvation, high and low temperatures, acidity and alkalinity, high pressures, and desiccation, to name the more relevant. In summary, most mineral environments with moisture and temperatures below $120^{\circ} \mathrm{C}$ can be expected to contain some microbial life.

A number of recent high-profile studies from deep ocean drilling programs have expanded our knowledge of the physiological types, extent, activities, and diversity of the bacteria and Archaea that growth at depth. ${ }^{29}$ This has enhanced our knowledge of their biogeochemical role and the extent of the biosphere. Some information on the terrestrial microbes at depth has come from microbial studies in deep mines and oil drilling wells. The former have confirmed microbes living at depths; in one case, the genome of a novel bacterium from a $2.8 \mathrm{~km}$ deep rock fracture was sequenced. ${ }^{30}$ The studies of microbes in oil wells have focused on the microbial role in well corrosion and oil field "souring." All such studies establish substantial and diverse microbial life at depth, but detailed information on the

\footnotetext{
${ }^{29}$ B.B. Jorgensen and S. D'Hondt. 2006. A starving majority deep beneath the seafloor. Science 314 : 932-934; J.S. Lipp, Y. Morono, F. Inagaki, and K.U. Hinrichs. 2008. Significant contributions of Archaea to extant biomass in marine subsurface sediments. Nature 454: 991-994.

${ }^{30}$ D. Chivian, E.L. Brodie, E. J. Alm, et al. 2008. Environmental genomics reveals a single-species ecosystem deep within Earth. Science 322: 275-278.
} 
indigenous microbes and their biogeochemical roles in rock environments are comparatively sparse.

One consistently high-profile area for biological advance is the discovery of new microbes that expand our knowledge of the strategies and limits of life, ${ }^{31}$ such as microbes that harvest new sources of energy, live at even higher temperatures or pressures, or exhibit new biochemical reactions, some of which may have biotechnological or pharmaceutical value. The discovery of these organisms often occurs in samples from unusual habitats where unique biology may have evolved. A facility for the described physics experiments would necessarily access subsurface material that could reasonably harbor unique biology, and the samples made available to the biological research community should be free from external chemical and microbial contamination. Important questions about the energy sources and energy efficiency of these organisms and about the evolution of small populations and horizontal gene exchange as well as mechanisms of mineral weathering could be addressed using the access enabled by the DUSEL physics facility. Other subsurface research facilities being put to use for the studies of microbes are the Ice Core Lab (http://nicl.usgs. gov) and the Integrated Ocean Drilling Program (http://www.iodp.org).

Sites dedicated to cross-disciplinary research in the biological and geosciences would be valuable. For example, phenomena where faults play a role are closely interconnected, but the disciplines that address them are in many cases not closely interconnected nor do they enjoy much professional interaction. Fluid transport and chemical reactions contribute to microbial life, and the microbes probably facilitate chemical reactions. Chemical reactions alter permeability and affect fluid pressures, which in turn may influence fluid flow and mechanical stability. Damage and flow conduits formed during an earthquake rupture can be healed, and resulting changes in permeability can be sealed by chemical reactions, thereby influencing subsequent fault slip. The rupture process itself may release hydrogen, carbon, or other compounds that go on to take part in chemical and biochemical reactions.

\section{Limitations}

All existing and proposed underground facilities have important limitations, especially for subsurface engineering and geoscience research. Many of the most interesting processes occur at depths and temperatures deeper and hotter than any of the proposed underground facilities. Moreover, all of the processes and interactions described earlier are sensitive to characteristics such as rock type, tectonic and structural setting, and rock history. DUSEL is in a specific geological setting,

\footnotetext{
${ }^{31}$ See H.N. Schulz, T. Brinkhoff, T.G. Ferdelman, H. Hernandez-Marine, A. Teske, and B.B. Jorgensen. 1999. Dense populations of a giant sulfur bacterium in Namibian shelf sediments. Science 284: 493-495.
} 
principally metamorphic rock in a low tectonic environment. It is, however, sedimentary rock (carbonates, sandstones, shales, etc.) that is the focus of a great deal of research because of its importance to oil and gas discovery and extraction, as well as the potential benefits associated with $\mathrm{CO}_{2}$ sequestration. Moreover, although many generic experiments can be conducted at Homestake, engineering applications may need to be demonstrated in specific rock formations. Yet, developing the tools to overcome scale and sampling challenges at an underground facility would have widespread impact.

This limitation applies to any single underground research site. Thus, research in subsurface engineering, geosciences, and biosciences (EGB) would benefit from international cooperation and a strategy of several subsurface sites. Owing to important variations in rock types, the investigation of loading conditions, temperature, and fluid regime at many sites is likely to yield the most valuable insights. Some of these sites need not be extensive long-term underground laboratories, since much information can be gained from targeted drilling.

\section{Experimental Details}

Several broad classes of EGB experiments have been described to date. All of these are intended to be accomplished over the first decade of DUSEL operations (i.e., 2014-2024):

1. Scale effects and coupled thermohydromechanical processes;

2. Subsurface imaging ("transparent Earth");

3. Modeling the mechanics of induced fracturing and fault slip; and

4. Biosciences

\section{Scale Effects and Coupled Processes}

Much of the research intended in this category was stimulated by the proposal to construct the large water Cherenkov cavity ( $\sim 60 \mathrm{~m}$ span) at a depth of $1.5 \mathrm{~km}$ $(4,850 \mathrm{ft})$. Such a cavity at this depth is unprecedented and would provide a unique opportunity for engineering research on the effects of (1) scale (both size and time) on rock deformation and (2) the preconditioning of rock mass (by blasting) to facilitate excavation and minimize damage to the final rock periphery. This experiment will require "halo" tunnels around the large cavity for instrumentation and monitoring. The dynamic response of various support systems installed in the halo tunnels could also be monitored during blasts as part of excavating the large cavern. Observation and characterization of fracture systems in the rock mass will be carried out in drifts (including in the halo tunnels) developed in preparation for the large cavern excavation. 
The complex coupled nature of thermal-hydraulic-mechanical-chemical (THMC) effects in subsurface systems is illustrated in Figure 3.10. The DUSEL experiment proposes to study the role of biological effects in such coupled processes, which may be significant in certain underground environments. Among the tests under consideration is a heated block test for studying THMC plus biological processes. The study proposes to heat a $50 \mathrm{~m} \times 40 \mathrm{~m} \times 40 \mathrm{~m}$ block of rock by an array of electrical heaters to a maximum temperature of between $150^{\circ} \mathrm{C}$ and $300^{\circ} \mathrm{C}$. The block will be delineated by two parallel drifts approximately 45 $\mathrm{m}$ (center to center) and a cross drift. Instrumentation will be deployed along the three drifts. Researchers will then study links between microbial activities, nutrient supply, biochemical reactions, and temperature. It is anticipated that this project will require approximately a decade to complete the heating and cooling phases.

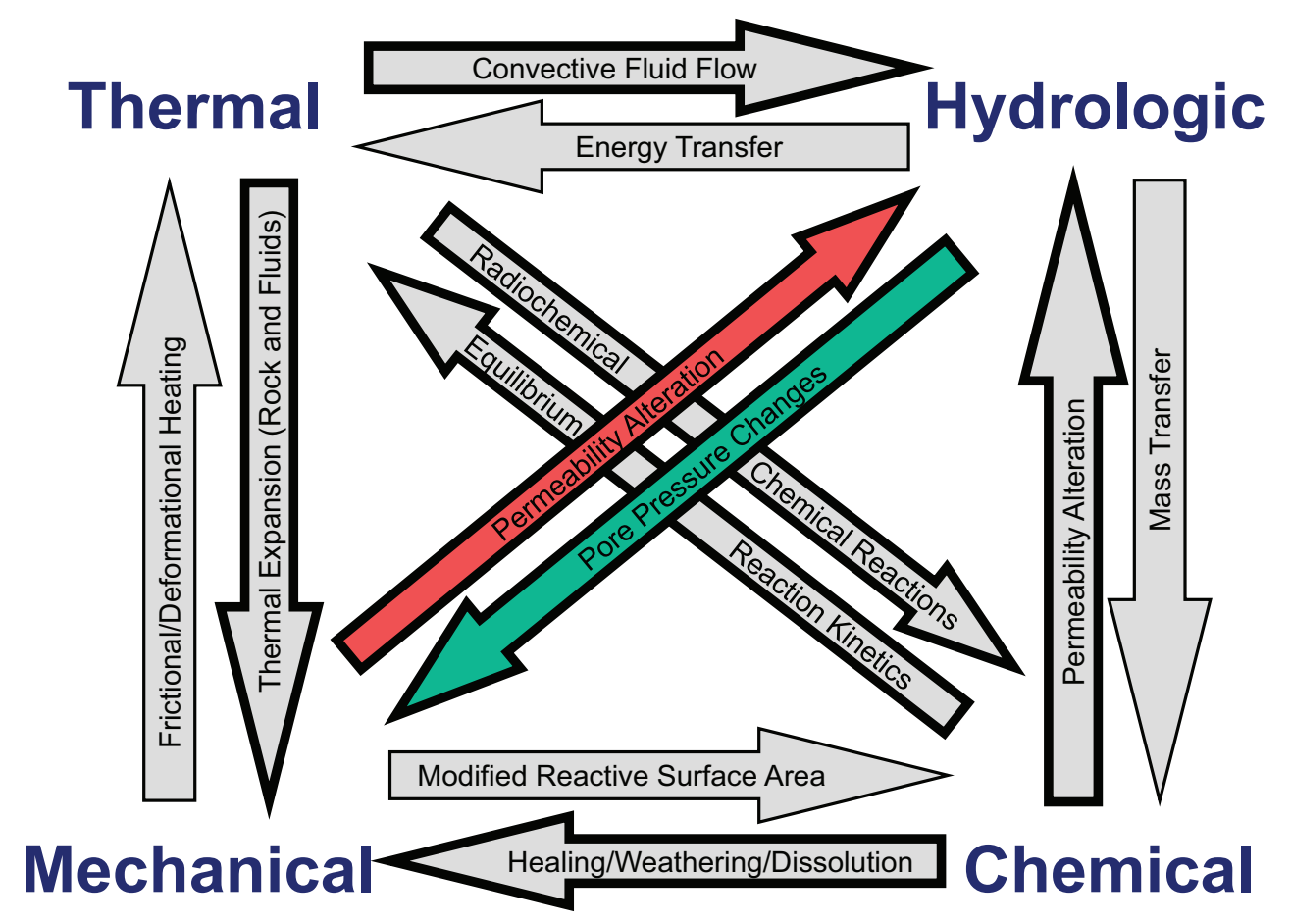

FIGURE 3.10 Coupled THMC (thermal-hydraulic-mechanical-chemical) effects in subsurface fluid flow systems. SOURCE: Adapted and reprinted from J.L. Yow and J.R. Hunt, Coupled processes in rock mass performance with emphasis on nuclear waste isolation, International Journal of Rock Mechanics and Mining Sciences, 39 (2): 143-150, copyright 2002, with permission from Elsevier. 


\section{Subsurface Imaging (Transparent Earth)}

The opacity of rock is a major impediment in subsurface engineering. The problems range from the inability to "see" a few tens of meters ahead of a tunnel boring machine to the precise location of "producing horizons" at depths of several kilometers and occur in petroleum extraction and in the search for ore deposits in mineral exploration. Experiments to explore the potential of a variety of geophysical techniques to make the rock more "transparent" are planned at DUSEL. Faculty from several universities are involved as a collaborative team, led by Steve Glaser at the University of California at Berkeley. Experiments include broadband and long-wavelength seismic arrays, passive electrical arrays, and electromechanical passive imaging. A rock block between two drifts 50 to $75 \mathrm{~m}$ apart is envisaged.

One problem limiting the wider applicability of imaging tests in hard-rock underground sites such as Homestake is that the geology is either highly complex (folded and faulted metamorphic rocks at Homestake) or markedly different from that in areas generally of interest to geoscientists (homogeneous granitic rocks versus sedimentary rocks). This might lessen the usefulness of imaging experiment results in these facilities for clarifying questions of widespread interest in the geosciences. Verification test results could be ambiguous or techniques developed at Homestake might not work elsewhere.

\section{Mechanics of Induced Fracturing and Fault-Slip Modeling}

Induced fracturing is a major element of much of subsurface engineering. Perhaps the most common example is massive hydraulic fracturing that is used extensively in the oil and gas industry. Recent applications in the United States to stimulate the extraction of geothermal energy and natural gas by fracturing have led, in some instances, to seismic tremors and proposed legislation to prohibit the use of fracturing. Other important methods of inducing fracturing include use of explosives and rock-cutting tools in tunnel boring machines, all in an effort to increase drilling rates in deep borehole drilling. ${ }^{32}$ A study has been proposed to conduct hydraulic fracturing tests in a rock block similar in dimension to the heated block test discussed in the preceding section. Instrumentation would be installed to detect microseismic activity and velocity changes during fracture propagation.

When a fault can no longer sustain the forces applied to it, dynamic slip may take place, resulting in earthquakes. A slip can occur from an increase in tectonic

\footnotetext{
${ }^{32}$ The rate of drilling (including rock removal) and the time spent in reaching the producing horizon directly affect the economics of offshore drilling. Energy costs for drilling are a small component of the overall costs of maintaining an offshore rig.
} 
loading, a decrease in fault slip resistance owing to hydrological, chemical, thermal, or other changes along the fault surface and in nearby rock, or to some combination of all of these factors. The spatial and temporal distribution of rock deformation leading to fault slip (and earthquakes) is inadequately known as are the processes that lead to and accompany progressive rupture. Many preexisting faults are believed to be active in today's stress field (critically stressed faults). ${ }^{33}$ If forces on faults are in a state of critical equilibrium the implications for engineering operations that disturb this equilibrium are profound. ${ }^{34}$ A deep underground laboratory could allow measurements of rock strain as a function of time and position near faults and in the rock mass. These data would help explain the influence of geology and human activity on strain and stress distribution in rock, allow observation of how deformation accumulates near faults and fractures, and provide insights into how laboratory and underground laboratory measurements of fault slip processes can be scaled to larger events. The understanding gained from this research could be a step toward reliable understanding of earthquake rupture processes and precursory phenomena.

At least two potential issues with these fault slip experiments should be noted. First, if experiments succeed in activating an instrumented fault, the ramifications for nearby physics experiments (and physicists) would need to be considered. ${ }^{35}$ This possibility might necessitate conducting the geoscience experiments during site construction, although this would result in the experiments operating on shorter than optimal timescales. However, since all of the proposed sites are in relatively tectonically quiescent areas, ${ }^{36}$ the second potential problem is that the experimental perturbations will be insufficient to cause an interesting response (i.e., there will be no earthquake). Selection of a site in a more seismically prone area or the application of an unfeasibly large perturbation might be needed before sufficient slippage will take place.

\section{Biosciences}

The proposed biology experiments fall into two categories: (1) those that seek to define and quantify the microbiological role in the rock weathering processes,

\footnotetext{
${ }^{33}$ C.A. Barton, M.D. Zoback, and D. Moos. 1995. Fluid flow along potentially active faults in crystalline rock. Geology 23(8): 683.

${ }^{34}$ For example, large-scale fluid injection for $\mathrm{CO}_{2}$ sequestration or hydraulic fracture water disposal could lead to widespread seismicity in otherwise tectonically quiescent areas.

${ }^{35}$ Efforts to prepare the Homestake mine for the physics experiments have included testing the structural capability of the surrounding rock and stabilizing and rehabilitating the space where needed. K.T. Lesko, Lawrence Berkeley National Laboratory, "Deep Underground Science and Engineering Laboratory (DUSEL) Project Overview," Presentation to the committee on December 14, 2010, p.11.

${ }^{36}$ However, in an otherwise tectonically quiescent area, numerous earthquakes occur near deep South African mines. Available at http://earthquakes.ou.edu/. Last accessed on September 29, 2011.
} 
including their contribution to the coupled THMC, and (2) those of a discovery nature that explore unknown aspects of biology provided by access to a unique habitat.

While the general capacities of microbes in rock weathering are known, their activities under field conditions - such as their natural rates, environmental controllers of those rates, biochemical mechanisms, and often the types of microbes themselves-are unknown. This information is important in quantifying the processes, their accurate modeling, scaling, and their integration into coupled processes. This gap in information is due largely to the lack of field laboratories at depth that would allow in situ studies under natural or nearly natural conditions. The reproduction of these natural conditions in a distant laboratory is currently impossible. While it may be possible to obtain the rock material, it is not possible to reproduce the natural water chemistry, including its natural redox state and flow conditions, or the indigenous microbial populations. Furthermore, the contamination of samples with external microorganisms during drilling and sample processing becomes a much greater problem in off-site studies. An additional advantage of field laboratory studies is that the site hydrology and geochemistry information can be directly integrated with the biological information. Defining and quantifying the microbial role in the coupled processes is the science area where important new biological knowledge should be reliably obtained in a DUSEL-like facility. The experiments planned for this area are well integrated with the nonbiological components and would greatly benefit from the data synergy that would occur.

Only one other underground microbiology laboratory exists in the world, the ASPO lab in Sweden. While it has proven the feasibility and value of such a lab at depth, it is small, fully used, only $400 \mathrm{~m}$ deep, and embedded in homogeneous granite, which offers only limited conditions for microbial study.

The second category of experiments would expand our knowledge of biology by (1) defining the depth of the biosphere and (2) determining whether some unique biology exists in terms of energy sources, physiology, and evolutionary outcomes, including life as we do not know it. In the first effort, the proposal is to drill deeper into the crust to determine where life ceases to exist-perhaps at the $120^{\circ} \mathrm{C}$ isotherm? The drilling cost would be reduced substantially since drilling could start from the existing excavations at 7,400 ft, the deepest directly accessible level in North America. This experiment would better define Earth's biosphere and biogeochemical inventory. However, while it would help fill gaps in our knowledge of the terrestrial biosphere, it would be costly relative to its potential science value. It is, after all, limited to a single location and the microbial densities are likely to be low, both of which are limitations compared to the proven value of ocean sediment studies.

The experiments for detecting novel biology are both intriguing and risky. The environment should select for novel energy specialists— "dark life," different 
evolutionary outcomes, and isolation from horizontal gene exchange with surface organisms - to name a few potential high-profile outcomes. But, the study is risky because the microbial density would probably be very low in this geologic material, conditions for microbial isolation might be difficult to determine, and the microbes might not have been isolated from the surface life for long enough to exhibit population or genetic differences. In evaluating the merits of this experiment, one must examine the extra cost for the biological objective in relation to the probable value of its results. The committee judges that undertaken alone, the experiment seems too costly. However, if the field lab and the THMC experiments are undertaken as well, then the extra cost of obtaining some biological samples is significantly decreased and at least some sample collecting would be warranted for these risky but potentially high-payoff experiments.

\section{Potential Future Lines of Inquiry}

The experiments proposed in the DUSEL program are only a fraction of the possible nonphysics studies that might take advantage of the existence of an underground research facility. Here, the committee presents a sampling of other promising lines of experimental inquiry. However, as noted in the preceding section, the value of the underground space for these experiments might depend on the types of rock present.

\section{Fracture Network Engineering}

The development and control of fracture networks at depth by remote stimulation of a rock mass is central to many aspects of subsurface engineering. Currently, although hydraulic fracturing is a major component of oil and natural resource development, it is still in some respects more art than science. It is a technology that is being applied increasingly to the development of other resources. In enhanced geothermal systems, for example, a fracture network is created at depth on the order of $6 \mathrm{~km}$ or more, where the rock temperature is approximately $300^{\circ} \mathrm{C}$ or higher. Water is circulated through the fracture system to extract heat. Cooling causes the rock to contract; fracture apertures change and hence also the pattern of circulation. Downhole-microseismic networks can monitor fracture development. It has been proposed to develop a model of the preexisting fracture network and develop a model of a stimulation plan, including predicted seismicity. The predicted activity can be compared with that observed and the stimulation procedure modified to improve the overall "heat exchange system." Such ambitious schemes will need to be tested, modified, and made robust before they can be applied successfully. FNE research experiments could be an excellent development of or supplement to the THMCB experiment proposed above. 


\section{Other Potential Future Experiments}

- Large-scale rock mechanics experiments, including induced brittle failure on new or preexisting natural faults through controlled stress relaxation (e.g., with slow release of hydraulic support structures) or other means.

- Seismic experiments to detect and monitor hydraulic fracture propagation and fault rupture with closely spaced monitoring devices and subsequent intense sampling or mining. An advantage of a dedicated site for such tests is that the dedicated site would not have the noisy active mining operations or nearby tunnels that are in use (traffic, water flow).

- Hydrogeologic experiments, including effects of microbes on flow properties. Such tests could include controlled flooding of deeper mine sections.

- Experiments relevant to nuclear and chemical waste disposal-for example, radioactive tracer studies.

- The underground access provided by DUSEL is an opportunity for determining some "ground truths" and improving three-dimensional seismic and other surface-based geophysical exploration techniques by comparing the geophysical predictions with actual observations at depth. Finally, the increasing variety of engineering applications of the underground-for example, for nuclear and hazardous waste isolation, including $\mathrm{CO}_{2}$ sequestration for the development of domestic natural gas resources, and for geothermal energy ${ }^{37}$ — will stimulate a variety of engineering studies for which DUSEL will be well suited.

Conclusion: The ability to perform long-term experiments in the regulated environment of an underground research facility could enable a paradigm shift in research in subsurface engineering and would allow other valuable experiments in the geosciences and biosciences.

\footnotetext{
${ }^{37}$ The events in Japan resulting from the devastating earthquake in 2011 have reopened discussion of underground location of nuclear reactors to avoid the possibility of releases of dangerous concentrations of radionuclides into the atmosphere.
} 


\section{4}

\section{Impacts of a National Underground Facility}

An underground research facility could be as simple as two or more colocated experiments that share infrastructure or as complete, wide-ranging, and integrated as the proposed DUSEL facility or anything in between. The committee's assessment of the impact of such a facility on the relevant research communities has so far been limited to a discussion of the importance of individual science questions and the scope and ability of the experiments in the program to address those questions. In any case, however, a facility's impact on these communities could extend beyond the particular experiments that have been discussed and even beyond these particular communities. For instance, a centralized and integrated infrastructure would not only support the experiments under consideration but also provide opportunities to pursue future research and support for the communities pursuing that research. A highly integrated facility such as the one proposed by the DUSEL program would also allow for educating the general public and benefiting nearby communities. This chapter discusses some of the impacts of such a facility.

\section{SHARED INFRASTRUCTURE AND INTEGRATED OVERSIGHT}

Conducting experiments underground requires substantial infrastructure and technical support. Co-location of underground experiments at a single site would 
enable researchers to efficiently share that infrastructure and support. ${ }^{1}$ The requirements include access to adequate space, power, ventilation, and ready ingress and egress. Safety is of utmost concern, because most of the proposed experiments have measurable safety risks, especially given that they may be conducted a mile or more underground. Because it is expensive to excavate and support underground space, almost all underground experiments would need laboratory space on the surface for assembling and maintaining apparatus as well as developing future work. There is typically also a need to set aside underground laboratory space for low radioactivity studies to reduce background signals. In addition, technical personnel are needed to maintain, operate, and manage the infrastructure. Much of this infrastructure and its associated staff could be shared by co-located experiments. The economies of scale could reduce construction and operating costs, although the extent of savings would need to be quantified by comparing alternate sites. The existence of a central location for underground physics research would also allow carrying out other science experiments.

Integration of infrastructure for co-located experiments would need to be accompanied by at least limited coordination. For example, an integrated safety program would be needed to ensure that all experimenters are safe from risks of their own and other experiments. The location of any given experiment at a site would also need to consider possible interference of other experiments during construction or operations.

While infrastructure could also be shared for some experiments by locating them here or abroad, this section considers the impact of a national facility. The mere colocation of experiments would give to the research communities some, but not all, of the advantages of a more integrated program, as described in the next section. Co-location alone would also not have such a broad impact on education and the public as would a national research facility similar to the proposed DUSEL program. ${ }^{2}$

\footnotetext{
${ }^{1}$ The term co-location is used to refer to two or more experiments located at a single site. For instance, the three main physics experiments could be co-located in the Homestake mine, or a dark matter experiment could be co-located with existing experiments at an existing laboratory. The proposed DUSEL program co-locates all of the experiments and foresees a more integrated program that includes additional aspects of a national laboratory, including a surface campus, a large user community, mechanisms for developing a future program, and an education/outreach facility.

${ }^{2}$ The committee notes that while the DUSEL program was developed with the expectation that the proposed experiments would be placed at the Homestake mine in Lead, South Dakota, the committee's conclusions on the advantages of co-locating experiments are not limited to that site and should still prevail, regardless of the site chosen. The balance of advantages and disadvantages of a site, including safety issues, will depend on the specifics of the site and experiments proposed to be installed at that site.
} 
The benefits of shared infrastructure and integrated oversight provided by co-location of experiments, as well as some of the benefits discussed in the next sections, lead to the following conclusions:

Conclusion: The co-location of the three main underground physics experiments at a single site would be a means of efficiently sharing infrastructure and personnel and of fostering synergy among the scientific communities. The infrastructure at the site would also facilitate future underground research, either as extensions of the initial research program or as new research initiatives. These additional benefits, along with the increase in visibility for U.S. leadership in the growing field of underground science, would be important considerations when choosing a site for the three main physics experiments.

Conclusion: If co-located with one or more of the main underground physics experiments in the United States, a small underground accelerator facility to enable measurements of low-energy nuclear cross sections important to nuclear astrophysics would benefit from shared infrastructure, personnel, and expertise.

Conclusion: In light of the potential for valuable experiments in subsurface engineering, the geosciences, and the biosciences that could be offered by an underground research facility, if such facility is constructed in the United States for physics experiments, scientists in other fields would greatly benefit by having a mechanism in place that would allow them to perform research there.

\section{STEWARDSHIP FOR THE RESEARCH COMMUNITIES}

One of the committee's tasks is to assess "the impact of the proposed program on the stewardship of the research communities involved." 3 Stewardship is a broad concept and can take many forms, although an essential aspect is that it provides a scientific community the opportunity to pursue its research. The degree to which a program provides stewardship for a community is reflected in the types of questions it allows the community to address, including how closely those questions are aligned with that community's strategic plans. That impact is enhanced by qualitative factors such as the intellectual atmosphere the program fosters; the ease with which the research can be pursued, including the proximity of the research site to researchers; the degree to which the program is able to meet future as well as

\footnotetext{
${ }^{3}$ Statement of Task, Appendix A.
} 
current research needs; and the opportunities and intellectual excitement that the program provides that allows it to attract and train not only the current generation of scientists but also the next one.

As described in Chapter 3, the physics program proposed for the DUSEL facility would address questions of tremendous scientific importance and so would have a strong, positive impact on the stewardship of the communities engaged in underground physics research. Further, those physics experiments play a prominent role in the long-range plans of the nuclear and particle physics communities and, hence, in the stewardship of these communities. The strategic plan prepared in 2008 by the Particle Physics Project Prioritization Panel (P5) of the High Energy Physics Advisory Panel (HEPAP) for the Department of Energy (DOE) and the National Science Foundation (NSF) emphasized the need for "a strong, integrated research program at the three frontiers of the field: the Energy Frontier, the Intensity Frontier and the Cosmic Frontier" 4 in order to answer the important questions in particle physics. The proposed DUSEL program would serve as a key element in this plan for both the Intensity Frontier and the Cosmic Frontier. The long-baseline neutrino oscillation experiment, coupled with a new high-intensity proton source at Fermilab, is a critical component of the Intensity Frontier program, along with neutrinoless double-beta decay experiment and the search for proton decay. Experiments aimed at the direct detection of dark matter are a critical component of the Cosmic Frontier program, which also includes the study of neutrinos from space, such as neutrinos arising from supernovas.

The most recent long-range plan of the Nuclear Science Advisory Committee (NSAC) for DOE and NSF identified the construction of DUSEL as "vital to U.S. leadership in core aspects" of the initiative to investigate neutrino properties and fundamental symmetries. ${ }^{5}$ This strategic plan for the nuclear physics field identified neutrinoless double-beta decay experiments and the discovery of neutrino properties through neutrino oscillation studies as core aspects of the field. The plan built on similar support for construction of DUSEL in the 2002 NSAC longrange plan, as well as recommendations in the NRC decadal study for elementary particle physics. ${ }^{6}$ The scientific programs foreseen in these strategic plans represent the prioritization of the science opportunities within each field, under budget assumptions provided by the agencies. Clearly, the realization of DUSEL is of high priority to both the nuclear and the particle physics communities and would have great positive impacts on the stewardship of those communities.

\footnotetext{
${ }^{4}$ DOE/NSF. 2008. US Particle Physics: Scientific Opportunities: A Strategic Plan for the Next Ten Years, Report of the Particle Physics Project Prioritization Panel, p. 2.

${ }^{5}$ DOE/NSF. 2007. The Frontiers of Nuclear Science: A Long Range Plan. Report of the Nuclear Science Advisory Committee, p. 7.

${ }^{6}$ NRC. 2006. Revealing the Hidden Nature of Space and Time. Washington, D.C.: The National Academies Press.
} 
The research communities involved in the proposed DUSEL program would include not only those engaged in particle and nuclear physics, but also segments of the biological, geological, and engineering science communities. The DUSEL program would give communities outside of physics the opportunity to perform valuable, long-term experiments in a regulated environment, thus advancing research potentials for those fields. Although important to research communities of all fields, an environment that offers robust research opportunity will be of great consequence for the subsurface engineering research community. As noted in a recent DOE report,

Chronic underinvestment in federal R\&D in ... [the engineering and geosciences] disciplines has eroded the nation's capacity to educate and train the next generation workforce necessary for industry, academia, and government.

As a result, the U.S. faces the prospect of ceding its historic leadership role in these disciplines, and thereby undermining its resource security. ${ }^{7}$

For this community, the opportunity to undertake scientific and engineering research experiments in situ in an underground facility such as the proposed DUSEL site would be critical in the effort to address these shortfalls. It would help revitalize programs in subsurface engineering at universities, allowing researchers to at long last move beyond small-scale tests on rock specimens and numerical modeling of rock mass behavior to the testing of theoretical concepts in the field. This, in turn, would give U.S. engineers the knowledge and skills to lead the sustainable development of subsurface resources.

Facilitating the development of future research opportunities is a one of the significant ways that the DUSEL program would provide ongoing stewardship of the research communities. As the proposed DUSEL evolves, it will implement a process for evaluating the merit of proposed future experiments. It can be expected that important underground experiments beyond the proposed initial DUSEL suite will emerge. For instance, the initial suite does not include all the experiments proposed in the course of developing the DUSEL design. These include efforts specialized to detect certain proton decay modes, solar neutrinos, or geoneutrinos, the last of which would constitute particularly interesting cross-disciplinary research. Nor does the initial DUSEL experimental suite include the next-generation physics experiments that can be expected to follow some of the initial ones. The committee was briefed on a future antineutrino oscillation experiment to measure the matterantimatter asymmetry in neutrinos and on future underground gravitational wave experiments. Moreover, for dark matter and/or double-beta decay studies, larger,

\footnotetext{
${ }^{7}$ DOE. 2009. Energy Research and Development (Document END09278): Strengthening Education and Training in the Subsurface Geosciences and Engineering for Energy Development, Section 33, Subtitle C, p. 3.
} 
more sensitive experiments or experiments that use different technologies may be required to either follow up on the observations of initial experiments or take fresh steps in case those experiments fail to reach the sensitivities needed to observe the desired events. DUSEL's planning process is intended to integrate selected experiments with the current suite of experiments, either by incorporating them into existing experimental space or by developing and implementing plans for excavating new space and expanding support services.

The biological, geological, and engineering science experiments described in the proposed program are simply representative of the interesting, and relatively modest, experiments that could also be staged at DUSEL in coming years. One may anticipate that many new concepts for experiments will emerge as the potential of DUSEL for underground research in these fields is realized and appreciated. Indeed, it is likely that, as in the past, the advantages afforded by the existence of the facility will suggest scientific opportunities far beyond those anticipated in the initial DUSEL program. As an example, the Amundson-Scott South Pole Scientific Station, originally developed for geophysics studies, created unique opportunities for astrophysics and cosmology.

The proposed DUSEL program, in bringing together particle and nuclear physicists at a single experimental site, will also provide a venue and program for interactions among scientists and an enriched, synergistic intellectual atmosphere that enhances their research. Having researchers from the biological, geological, and engineering sciences conduct experiments alongside physicists may result in the application of experimental techniques to new fields just as, in the past, the rapid development of particle astrophysics and dark matter experiments relied on techniques and facilities developed to support accelerator- and space-based experiments.

In practice, it is more convenient, easier, and less costly in terms of travel expenses for U.S. research communities to perform experiments at a single location such as the proposed DUSEL site. Such a site would also make it easier to attract students and postdoctoral researchers to a research program, to allow faculty to more efficiently split their time between teaching and research commitments, and to give all researchers the chance to optimize their time at their experimental site. Indeed, the siting of at least some research facilities on U.S. soil is essential to a vital and healthy scientific program.

By providing underground research space and a planning process for realizing meritorious underground science, the proposed DUSEL program will afford individual U.S. scientists and research groups a context in which they can pursue their concepts. This context, together with the exceptional scientific opportunities planned, will help ensure that they attain their full intellectual and research potential. It may also smooth the way for U.S. scientists to maintain the intellectual leadership roles that they have played in underground science, particularly in 
the face of the growing scale of experiments, increased demand for underground laboratory space, and shrinkage in the numbers of experiments tackling certain critical science questions.

On the larger international stage where the U.S. nuclear and particle physics communities play their part, the proposed DUSEL program, coupled with the U.S. Intensity Frontier program based on the Fermilab accelerator complex, would give the United States the leadership role in a field of great scientific interest over at least the next two decades, when research at the Energy Frontier will be led by the European Large Hadron Collider. Such a leadership role would contribute significantly to the sense of scientific opportunity needed to steward these communities. Indeed, the proposed DUSEL program, being world class, would be able to attract considerable international interest and participation, which, in turn, would enhance the quality of research at the facility and the intellectual environment.

The research environment provided by DUSEL would offer an ideal venue for training students and postdoctoral scholars in research, with the international context contributing to the value of that training. The importance of the science, the vibrant research environment, and the intellectual opportunity on frontier science would all help attract talented young students into the science and technology workforce.

The committee recognizes that the proposed DUSEL program would not be the only way to provide stewardship to these research communities. For instance, as discussed more fully in the following section, other underground laboratory space is available and new underground research space can be created in other contexts. Research training and interdisciplinary interactions could also be provided in other contexts. Nevertheless, as outlined above, the proposed DUSEL program would provide strong stewardship to the research communities in several different ways. It would be much more extensive than the simple co-location of experiments and would provide many advantages to the research communities that are not available in a limited facility where experiments are simply co-located. Moreover, the proposed DUSEL program is intended to complement, rather than duplicate, existing facilities or their experiments. While the final decision on whether a national underground facility should be built should take into account many other factors, including the programmatic goals of the funding agencies and the financial costs of different options, significant advantages would accrue to the U.S. research communities involved in these research areas were such a facility be sited in the United States.

Conclusion: A facility for underground research would have a significant positive impact on the stewardship of the research communities involved. Such a facility would offer the particle and nuclear physics communities access to the underground research space they need to undertake a range of 
scientifically critical experiments, and it would allow the bioscience, geoscience, and subsurface engineering communities to perform valuable longterm experiments in a regulated environment.

\section{NEED TO DEVELOP SUCH A PROGRAM IN THE UNITED STATES}

In addition to providing stewardship of research communities, other factors are relevant for assessing whether to develop the proposed DUSEL program, or components of that program, in the United States. Some of these factors are specific to a given experiment and others are of a more general nature. Those factors are discussed in this section.

\section{General Considerations}

While the United States is uniquely well positioned for mounting the longbaseline neutrino oscillation experiment, its ability to mount the other physics experiments in the proposed DUSEL program is not unique. These experiments, which do not need a beam from an external accelerator, could, in principle, be mounted in an underground research facility anywhere in the world. Nonetheless, there are good reasons to mount these experiments in the United States. General considerations pertaining to mounting experiments in the United States rather than abroad include the availability of suitable laboratory space, efficient approval procedures, cost savings, partner commitment, and recognition of the U.S. role.

Laboratories in other countries, most notably at Gran Sasso in Italy and at the Sudbury Neutrino Observatory Laboratory (SNOLAB) in Canada, and possibly at the proposed Chinese Underground Laboratory at some future date, appear to have some space where experiments could be sited. However, in order to be placed there, the experiments proposed for the DUSEL program would need to compete for space with experiments from the host nations. Competition would include experiments that are part of the worldwide program for tackling some of the same research questions - for instance, from the dark matter and neutrinoless doublebeta decay programs, both of which call for multiple experiments. Unfortunately, it is difficult to foresee how much space might be available abroad for future U.S.-led experiments, because future programs or expansions of overseas laboratories are not yet well defined.

A U.S.-led experiment at a foreign laboratory would need to be approved by the host laboratory, with some associated uncertainties. In order to gain approval, U.S. scientists would need to submit a proposal to the laboratory that would then be reviewed by its program or scientific advisory committee, with approval dependent not only on the scientific merits of the proposal but also on how the proposed project fits within the overall objectives of the laboratory. If no space is available 
abroad for a U.S.-led experiment, the United States could pay for the excavation of a new space in a foreign underground laboratory. However, obtaining the necessary approval for such excavation is usually very difficult, and the excavation could be quite costly.

Some foreign underground research facilities that might be considered candidates for a U.S.-led experiment such as SNOLAB are in operating mines, which presents a separate set of issues. There are trade-offs to be considered in choosing between an experimental site at a dedicated research facility and one in an operating mine. On the one hand, ongoing mining operations usually absorb some of the rather large costs of access for underground research. On the other hand, research in an operating mine is subject to the interests of the mine operator and to its continued agreement to support research in the mine. Considering that some of the lines of research for the proposed DUSEL program, such as dark matter and double-beta decay, will probably be carried on for decades, and that some of the experiments require many years to mount, the need for the continued agreement of the mine owner and the risk that economic conditions might cause the owner to cease operating the mine, contribute uncertainty to constructing and conducting an experiment in an operating mine. Issues of liability and decision making in the event of an accident present additional complications.

These insecurities counterbalance at least some of the achievable cost savings associated with such a facility, so that the cost savings by funding an experiment at a foreign site must be determined on a case-by-case basis. Although enhancing a foreign underground laboratory might be less costly in the short term, the uncertainties surrounding space and approvals might favor a U.S. facility, which could ensure or at least facilitate the participation of U.S. scientists, who have historically been leaders in underground science. Furthermore, if significant discoveries are made by a U.S.-led experiment abroad, much of the recognition would go to the host country despite the U.S investment.

To summarize, in order for a foreign site to be deemed appropriate for a U.S.funded experiment, a number of conditions should be satisfied. In addition to adequate space, infrastructure, and other technical criteria, there should be clear, well-established cost savings advantages over a U.S. site. Furthermore, it would have to be understood that the important scientific programs-for instance, in dark matter and double-beta decay-would probably take decades. A foreign site would therefore have to guarantee continuing access, the long-term sharing of operating costs, and the ability and permission to expand laboratory space in the future. Such assurances will probably call for high-level agreements between governments and private corporations if operating mine sites are used. Furthermore, because it is important that the U.S. science achievements be recognized, it must be clear that a U.S.-funded experiment is part of the U.S. science program, despite the participation of international partners. 


\section{Specific Considerations}

In addition to the general considerations, the experiments in the proposed DUSEL program have specific considerations that should be taken into account in determining whether to install those experiments at a U.S.-based facility or abroad.

\section{Neutrino Oscillation Experiments}

Although design studies for a very large neutrino detector are currently under way in Japan (Hyper-Kamiokande), in Europe (LAGUNA), and in China, a large neutrino oscillation experiment in the United States could be coupled with the present and future capabilities of the Fermilab accelerator complex to provide an intense neutrino beam at a suitably long baseline. No other region can currently offer a fully competitive combination of an intense neutrino source and an appropriate underground laboratory site for a very large neutrino detector. No existing underground laboratory has space for such an enormous detector, so a new large underground cavern would have to be excavated wherever the experiment is built. Furthermore, the huge cost of such an ambitious detector and cavern makes it fairly unlikely that more than one such experiment would be built. Thus, mounting the long-baseline neutrino experiment here would allow the United States to lead the world in neutrino physics, as well as serve the world neutrino physics community. Moreover, if a second large neutrino detector were someday built elsewhere in the world, the programs here and abroad undoubtedly would be designed to be complementary and not duplicative. Different experimental techniques would allow important cross-checks of delicate, sensitive measurements. Different detector technologies-for example, water Cherenkov and liquid argon-might be used, and the combination of neutrino energy and baseline would probably be different. In addition to affording cross-checks, different baselines would have different "matter effects," which would help untangle contradictions between the mass hierarchy and the charge-parity (CP) angle, $\delta$. In addition, results from two experiments could be combined to improve sensitivity to small effects in these delicate experiments.

\section{Dark Matter and Double-Beta Decay Experiments}

The considerations for mounting the experiments in direct detection of dark matter and neutrinoless double-beta decay are similar. They include the need for multiple experiments and for deep sites and/or large caverns, as well as an acknowledgement of the global nature of these programs.

There is general recognition everywhere that at the present stage of these programs and probably also at the next stage, multiple, complementary experimental efforts using diverse techniques are needed. Complementary experiments 
are needed because a particular technique may prove most effective-for instance, for background suppression. Moreover, multiple techniques will provide essential cross-checks if a signal is detected (see Chapter 3). Competition and diversity will increase the likelihood of success for these extremely important efforts. When the results of two or more experiments are combined, the overall sensitivity to these exceptionally rare occurrences will be increased. Even if discovery claims are made in the next few years, independent confirmation will be needed using a wide variety of techniques, including different target nuclei in the case of the dark matter experiments or different isotopes for the neutrinoless double-beta decay experiments. In the case of double-beta decay, if and when a signal is detected measurements must be made with multiple isotopes to differentiate between the quantitative effects of nuclear matrix elements and neutrino mass. As these international programs evolve, it becomes reasonable to expect that hosting and supporting future large experiments would be shared by underground laboratories in different countries. U.S.-based dark matter and double-beta decay experiments will be part of the required complement of experiments needed in both fields.

Relatively few underground sites would at present be able to host a large thirdgeneration dark matter or double-beta decay experiment. These experiments call for deep sites, although background mitigation mechanisms (large water shields, neutron vetoing) may be possible. In that case, the shielding structures will necessarily be thicker and require correspondingly larger experimental halls (say, about $20 \mathrm{~m}$ ). These space needs mean fewer underground sites would be available for hosting these experiments, making a case for excavating new caverns.

The size and complexity of future dark matter and double-beta decay experiments, the potential scarcity of specific target materials for dark matter experiments and of specific nuclear isotopes for double-beta decay experiments, and the substantial costs of the experiments will probably necessitate global collaborations to amass the effort, material, and financing required. As stated previously, at least two dark matter experiments and at least two double-beta decay experiments are needed worldwide to successfully address these two key scientific questions. Realization of the required experiments depends upon the existence of appropriate underground sites to host them. If it fails to provide such a site to host some of these experiments, the United States will be abdicating its share in these critical discovery programs, as well as missing an opportunity to provide stewardship of them, ensuring opportunities for U.S. scientists to become involved in these programs in a major way, and ensuring continued U.S. leadership.

\section{Proton Decay and Supernova Neutrinos}

Searches for proton decay and for neutrinos from supernovae would also benefit from multiple experiments. For instance, in proton decay, sensitivity depends 
on detector mass. Therefore, multiple experiments would be complementary simply by combining their results. Furthermore, multiple experiments using different technologies could enable greater advances on many fronts. For instance, different detector technologies (i.e., water Cherenkov and liquid argon) have different sensitivities to different proton decay modes. Adopting variations in technique, such as gadolinium doping or different segmentation or photodetector coverage, might increase sensitivity to different ranges of neutrino energy, which would provide further complementarity. To the extent that the search for proton decay is background limited, multiple techniques might enable greater sensitivity as well as provide cross-checks that would be critical if a signal is detected. Finally, if supernova neutrinos are detected, multiple observations would be needed as cross-checks for this very rare event.

\section{Nuclear Astrophysics Experiment}

While the cross-section measurements to be made by the nuclear astrophysics accelerator experiment are delicate and challenging, they are not as scientifically uncertain as the searches for dark matter and neutrinoless double-beta decay. Nevertheless, having more than one nuclear astrophysics facility in the world would be of scientific value, particularly because the number of cross-section measurements to be made in order to determine astrophysical processes is more than the existing LUNA facility at the Gran Sasso laboratory can accomplish on its own. The proposed DIANA facility will have much to measure; moreover, it has reduced backgrounds with respect to LUNA, making it capable of producing measurements more quickly and with improved results. The two facilities together will more rapidly complete the set of fundamental measurements needed to elucidate important astrophysical processes.

\section{Experiments in Subsurface Engineering and the Geosciences and Biosciences}

For subsurface engineering, the geosciences, and the biosciences, special considerations include characteristics of the proposed DUSEL program as well as the need for multiple research environments. Existing underground research laboratories around the world for subsurface engineering were all developed for studies related to radioactive waste isolation. They are all relatively shallow (ca. $500 \mathrm{~m}$ ) and none are comparable to DUSEL in size or in scope. DUSEL would provide the opportunity for a broad range of experiments directed at a general understanding of the subsurface and enabling validation of computer modeling. In situ rock is a complex system. It is affected by tectonic forces and unstable slip events, including earthquakes, and contains networks of fractures that have a profound influence on rock deformability and strength and that serve as pathways for groundwater 
flow. Sophisticated numerical models are now available to describe this system over a wide range of temporal and spatial scales, but further advances depend on experimental verification of the model predictions. The regulated environment of DUSEL would offer this opportunity. For the first time, it would be possible to define the engineering characteristics of the subsurface. This ability would have profound and far-reaching implications for the wide variety of engineering activities involving the subsurface, and for this reason DUSEL is attracting considerable international interest.

However, an individual underground research facility basically provides only a single geological and biological environment for study. The development of multiple underground facilities around the world for geological and biological observation and experimentation would overcome this limitation, enable complementary experiments, and provide the ability to compare and contrast observations in different environments. Furthermore, the site proposed for the DUSEL program offers some special features that would be attractive for particular subsurface engineering experiments. Considerations related to revitalization of the U.S. academic subsurface engineering community (as discussed previously) are of particular relevance to the proposed DUSEL program.

\section{Summary of the Need}

As suggested above, a national underground research facility in the United States would provide the world with much-needed laboratory space for experiments in the burgeoning international field of underground science. A growing, widespread appreciation of the importance of the scientific questions that can be addressed by underground experiments and the need for large underground laboratories for the next-generation experiments with the sensitivities needed by the science drive the demand for increased underground laboratory space. Moreover, in order to address the experimental challenges of this important science, multiple experiments are needed worldwide to address each major science subject. The United States has a role to play in this flourishing field by not only providing leadership in particular areas or in the field as a whole but also hosting a portion of the ambitious worldwide program that is required to address these challenging science questions. Finally, a U.S.-based underground research facility would reinforce stewardship of the U.S. research communities by providing a research site that does not involve distant or international travel and that would facilitate graduate student training.

In summary, a number of reasons exist for developing an underground facility in the United States. As discussed in the preceding section, some of these reasons relate to the stewardship of research communities. In addition, a long-baseline neutrino experiment located in the United States would benefit from the combination 
of an intense Fermilab neutrino source and a suitably long baseline between source and detector site. For other experiments, there are general considerations related to the global availability of appropriate laboratory space for underground experiments and to access to foreign laboratory space for U.S. experiments. There are also a number of reasons specific to certain experiments. For instance, the global research programs in direct detection of dark matter and neutrinoless double-beta decay each require at least two experiments somewhere in the world. There are also reasons relating to co-location of experiments, discussed in a preceding section. An underground facility would provide a venue for future underground experiments as well as the initial set. The final reason is the benefits of a major U.S. role as a partner in the expanding field of underground research.

Conclusion: Development of an underground research facility in the United States would supplement and complement underground laboratories around the world. A U.S. facility could build upon the unique position of the United States that would allow it to develop a long-baseline neutrino experiment using intense beams from Fermilab. It could accommodate one of the large direct detection dark matter experiments and one of the large neutrinoless double-beta decay experiments that are needed by the international effort to delve into these critical scientific issues, while sharing infrastructure among the three experiments, which are of comparable import. It could also host and share infrastructure with other underground physics experiments, such as an accelerator to study nuclear astrophysics, and with underground experiments in other fields. An underground research facility would benefit the U.S. research communities, and would guarantee the United States a leadership role in the expanding global field of underground science.

\section{BROADER IMPACTS}

In addition to providing stewardship of the involved communities, other factors pertinent to the assessing the value of the DUSEL program include its positive effect on raising the visibility of scientific accomplishments of U.S. scientific communities and the opportunities it offers to provide education and outreach to the general public.

\section{Visibility of the U.S. Scientific Accomplishment}

A national underground research facility such as the proposed DUSEL would also advance more general national interests. It is well recognized that a vigorous and visible national scientific research program will significantly contribute to the 
future health of the U.S. economy. The 2007 NRC study Rising Above the Gathering Storm: Energizing and Employing America for a Brighter Economic Future ${ }^{8}$ argues persuasively that the long-term economic health of the United Sates depends critically on maintaining a strong scientific and technical base. The study presented four recommendations: increasing America's talent pool by vastly improving K-12 science and mathematics education; sustaining and strengthening the nation's commitment to long-term basic research that has the potential to be transformational; making the United States the most attractive setting in which to study and perform research; and ensuring that the United States is the premier place in the world to innovate. A strong program of deep underground science with a number of cutting-edge experiments at a single location would be a powerful focus for activities that address these recommendations. The excitement of the scientific program and the potential for discoveries that would fundamentally change our description of the world would attract world-class scientists and help maintain the preeminent role the United States plays in scientific research. Further, the technical challenges associated with an underground facility would lead to the development of innovative engineering techniques and provide a focus for engineering research.

\section{Educational and Outreach Opportunities}

The science to be explored by the proposed DUSEL program is world-class and transformative, highlighting experiments that aim at understanding the basic nature of the Universe. Any such program will typically attract and excite students at all levels and the public at large and would provide excellent opportunities for science education. The current and proposed education and outreach program at Sanford Laboratory is an excellent model of what the educational component of an underground research facility could provide. The program has two main goals: enhancing the understanding of science at all levels and bringing scientific education to historically underrepresented groups. In particular, the geographical location of the proposed DUSEL facility would bring science to Native Americans. The compelling nature of the science and the unique opportunities of an underground research facility would inspire students and prepare them for careers in technical and scientific fields. Summer research programs would bring high school teachers and students to the site and provide a hands-on chance to do research. Collaborations with local universities would help those universities recruit and retain faculty and expand their programs in physics and engineering. Components of such a program might include the following:

\footnotetext{
${ }^{8} \mathrm{NAS}, \mathrm{NAE}$, and IOM. 2007. Rising Above the Gathering Storm: Energizing and Employing America for a Brighter Economic Future. Washington, D.C.: The National Academies Press.
} 
- Internships for high school and college teachers to allow them to contribute to the scientific output of the experiments and to help them craft innovative educational programs for their students.

- Summer programs for high school and college students to encourage them to consider careers in science.

- Outreach programs where top-notch scientists travel out to the community to work with K-12 school groups. These programs could have especially important effects on underrepresented communities.

- Collaborations with faculty at local colleges and universities

An underground research facility such as the proposed DUSEL laboratory would take advantage of the public's curiosity about what lies below Earth's surface to attract them to the facility, where they can be exposed to frontier science and state-of-the-art technology. The Soudan Underground Laboratory has been successful in attracting the public. Of course, safety is important, so visitors would have to be well protected if outreach programs take them underground. 
Copyright (C) National Academy of Sciences. All rights reserved. 


\section{Appendixes}


Copyright (C) National Academy of Sciences. All rights reserved. 


\section{A}

\section{Statement of Task}

The committee will undertake an assessment of the proposed DUSEL program, including:

- An assessment of the major physics questions that could be addressed with the proposed DUSEL and associated physics experiments,

- An assessment of the impact of the DUSEL infrastructure on research in fields other than physics,

- An assessment of the impact of the proposed program on the stewardship of the research communities involved,

- An assessment of the need to develop such a program in the United States, in the context of similar science programs in other regions of the world, and

- An assessment of broader impacts of such an activity, including but not limited to education and outreach to the public. 


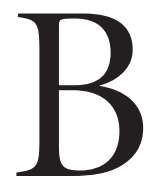

\title{
Meeting Agendas
}

\author{
FIRST MEETING \\ WASHINGTON, D.C. \\ DECEMBER 14-15, 2010
}

Tuesday, December 14, 2010

9:00 a.m. Welcome

Perspectives from the National Science

Foundation (NSF)/Division of Physics

9:30 Break

9:45 Perspectives from the NSF/Division of

Mathematics and Physical Sciences (MPS)

10:15 Perspectives from the Department of Energy

(DOE)/Office of High-Energy Physics (HEP)

10:45 Discussion with support agencies

11:00 DUSEL project overview
Andy Lankford, Chair

Joe Dehmer, NSF

Ed Seidel, NSF

Dennis Kovar, DOE
Kevin Lesko, University of
California at Berkeley

(UCB) 
12:15 p.m. Lunch break

1:00 Science Presentations, long-baseline neutrinos

Bill Marciano,

Brookhaven

National Laboratory

1:40 Science Presentations, PDK and other physics Bob Svoboda,

University of

California at Davis

2:20 Science Presentations, dark matter

Bernard Sadoulet,

UCB (via audio/

video)

3:05 Break

3:20 Science Presentations, biology

T.C. Onstott,

Princeton University

3:50 Science Presentations, geoscience and Derek Elsworth, engineering

Penn State

University

4:40 Science Presentations, nuclear astrophysics

Michael Wiescher, University of Notre

Dame

5:10 Science Presentations, double-beta decay

Steve Elliott, Los

Alamos National

Laboratory

5:55 Meeting closes

Wednesday, December 15, 2010

8:00 a.m. Welcome

8:00 Perspectives from the DUSEL Program Advisory Committee (PAC), physics

8:30 Perspectives from PAC, biosciences, geosciences, and engineering
Andy Lankford, Chair

Mike Witherell, University of

California at Santa

Barbara

Mark Zoback, Stanford University 
9:00 Perspectives from Fermilab

9:45 Break

10:00 Perspectives from the National Science Board
Pier Oddone, Fermilab

Barry Barish, California Institute of Technology

\section{SECOND MEETING}

IRVINE, CALIFORNIA

FEBRUARY 3-4, 2011

\section{Thursday, February 3, 2011}

Closed Session

Open Session

9:00 a.m. Welcome

Andy Lankford,

Chair

9:00 International aspects

Eugenio Coccia, University of Rome

Tor Vergata

10:00 Break

10:15 Neutrino target, beam-line issues

Vaia Papadimitriou, Fermilab

11:00 LBNE technical challenges

Jim Strait, Fermilab

11:30 Questions and answers on LBNE

Noon Lunch break

12:45 p.m. Science Presentations, geoscience/ geoengineering, dewatering, and DUSEL Research Association (DuRA)

1:30 Science Presentations, geoscience/ geoengineering, faulting studies

Larry Murdoch, Clemson University

Leonid

Germanovich, Georgia Tech University 
2:15 Questions and answers on geoscience/ geoengineering

2:45 Break

Closed Session

Friday, February 4, 2011

Closed Session

\author{
THIRD MEETING \\ IRVINE, CALIFORNIA \\ MARCH 25-27, 2011
}

Friday, March 25, 2011, through Sunday, March 27, 2011

Closed Session 


\section{C \\ Biographies of Committee Members}

Andrew J. Lankford, Chair, is professor in the Department of Physics and Astronomy at the University of California at Irvine. He received both his undergraduate and graduate education at Yale University, receiving his Ph.D. in 1978. After serving as staff scientist at the Lawrence Berkeley Laboratory and the Stanford Linear Accelerator, Dr. Lankford joined the faculty at the University of California at Irvine in 1990 and served as department chair from 2002 to 2007 . He is a fellow of the American Physical Society. His current research activities are in elementary particle physics and include a leading role in the ATLAS collaboration at CERN. Dr. Lankford is the deputy spokesperson for ATLAS and is on its executive board. ATLAS is an experiment being performed at the Large Hadron Collider by a collaboration of more than 3,000 physicists and engineers participating from 174 universities and laboratories in 38 countries. Dr. Lankford has served on numerous advisory and assessment committees and was on the NRC Committee on NASA's Beyond Einstein Program: An Architecture for Implementation.

Yoram Alhassid is professor of physics at the Center for Theoretical Physics, Yale University. He is an accomplished theoretical physicist who has made numerous contributions and has broad expertise in the fields of many-body nuclear theory, mesoscopic physics, and nanoscience. In particular, he has developed novel methods for understanding the statistical properties of quantum many-body systems, including atomic nuclei and quantum dots. Dr. Alhassid received the Aharon Katzir prize, awarded to one doctoral recipient for excellence in natural sciences in Israel. 
He was a Chaim Weizmann fellow at the California Institute of Technology and was awarded an Alfred P. Sloan Foundation fellowship in physics. He is a fellow of the American Physical Society and the recipient of an Alexander von Humboldt Senior Scientist Award, given for his contributions to many-body theory in nuclear and mesoscopic physics. Dr. Alhassid was a scientific director of two programs at the Max Planck Institute, Dresden, Germany: "Dynamics of Complex Systems" and "Electrons in Zero-Dimensional Conductors: Beyond the Single-Particle Picture." He served as the lead organizer of two interdisciplinary summer programs at the intersection of nuclear physics and condensed matter physics at the Institute of Nuclear Theory, University of Washington, Seattle: "Chaos and Interactions: From Nuclei to Quantum Dots" and "From Femtoscience to Nanoscience: Nuclei, Quantum Dots and Nanostructures.” Most recently he served on a review panel for the DOE Innovative and Novel Computational Impact on Theory and Experiment (INCITE) program, reviewing proposals in nuclear, particle, and cold atom physics. Dr. Alhassid has published over 200 papers.

Eugenio Coccia is professor of astronomy and astrophysics at the University of Rome, "Tor Vergata." He is a distinguished experimental physicist with expertise in astroparticle physics, with a focus on the detection of gravitational waves, as well as interests in cryogenic experiments for neutrinoless double-beta decay and in the development of detectors of cosmic rays. For 6 years Dr. Coccia directed the Gran Sasso Laboratory of the Italian National Institute of Nuclear Physics (INFN). He has been chair of the Astroparticle Physics Scientific Committee of INFN and president of the Italian Society of General Relativity and Gravitational Physics. Dr. Coccia is a member of the International GRG Society, the Italian Physical Society, the IUPAP-affiliated Particle and Nuclear Astrophysics and Gravitation International Committee (PANAGIC), and the Gravitational Wave International Committee (GWIC). He has been chair of the Coordination Management of European Underground Laboratories (CoMag), and a member of the European Committee for Future Accelerators (ECFA), of the CERN Strategy Group on Particle Physics, and of the OECD Astroparticle Physics working group of the Global Science Forum.

Charles Fairhurst, Itasca Consulting Group, Inc., (NAE) is professor emeritus in the Department of Civil Engineering at the University of Minnesota. Dr. Fairhurst received his education at the University of Sheffield, England. His current research specialties are the mechanics of rock fracture and rock masses, the determination of in situ stresses in rock masses, and rock mechanics and related technical issues in the geological isolation of high-level nuclear waste. Dr. Fairhurst served as president of the International Society for Rock Mechanics (1991-1995) and as a member of the board of governors for the Geo-Institute of the ASCE (19982001). Among his many awards are membership in the Royal Swedish Academy of 
Engineering Sciences; the AIME Outstanding Achievement Award, Rock Mechanics; the Pergamon Medal from the American Underground Space Association; and honorary degrees from the National Institute of Lorraine, France, and the St. Petersburg Mining Academy and Technical University, Russia. Dr. Fairhurst has served on a number of National Academies committees and studies and as the NAE Section 11 Liaison until October 5, 2010.

Bradley W. Filippone is professor of physics at the California Institute of Technology. His research focus is experimental nuclear physics and has included nuclear astrophysics and high-energy electron scattering from nucleons and nuclei. Recently he has focused on physics beyond the Standard Model in free neutron beta decay and on the search for the neutron's electric dipole moment. He is a fellow of the American Physical Society and has served on the NRC Committee on Theory and Laboratory Astrophysics (1989-1990) and the NRC Committee on Nuclear Physics (1996-1998), as well as on numerous review panels for the National Science Foundation and the Office of Science of the Department of Energy.

Peter Fisher is professor of physics and division head of the Massachusetts Institute of Technology's Particle and Nuclear Experimental Physics Division. He received a B.S. in engineering physics from the University of California at Berkeley in 1983 and a Ph.D. in physics from the California Institute of Technology in 1988. His research focuses on the experimental detection of dark matter using a new kind of detector with directional sensitivity. His other projects include neutrino physics, wireless power transfer, pedagogical work on electromagnetic radiation and development of new kinds of particle detectors.

Takaaki Kajita is director of the Institute for Cosmic Ray Research at the University of Tokyo and a principal investigator at the Institute for the Physics and Mathematics of the Universe. His research mainly involves participation in the Kamiokande and Super-Kamiokande experiments studying atmospheric neutrinos and neutrino oscillations. Among his many awards and honors are the Asahi Prize (as a member of the Super-Kamiokande collaboration) for the study of the neutrino mass, the Nishina Memorial Prize for the discovery of the atmospheric neutrino anomaly, and the American Physical Society's W.K.H. Panofsky Prize in Experimental Particle Physics.

Stephen E. Laubach is a senior research scientist and the Jackson Research Excellence Fellow at the Bureau of Economic Geology, Jackson School of Geosciences, University of Texas at Austin. He is a geologist with expertise in structure, fractures, diagenesis, fluid flow, and rock mechanics, as well as chemical and mechanical interactions in the deep subsurface. Dr. Laubach was awarded the Jules Braunstein 
Memorial Award from the American Association of Petroleum Geologists in 1999 for research on novel methods to assess fractures in the subsurface. He served as a member of the NRC Committee on Advanced Drilling Technologies from 1992 to 1994. He co-chaired the first North American Rock Mechanics Symposium in 1994. Dr. Laubach was a distinguished lecturer for the Society of Petroleum Engineers in 2004 and a member of the Geological Society of America Panel on Energy and Mineral Resources Policy in 2007 and 2008. He is the elected editor of the American Association of Petroleum Geologists and a member of its executive committee.

Ann Nelson is a professor of physics at the University of Washington studying elementary particle theory. Her current research interests include the theory and phenomenology of physics beyond the Standard Model. Some of the principal consequences of such a theory would be explored in several of the experiments being considered at DUSEL, including neutrino oscillation, dark matter, and neutrinoless double-beta decay studies. Among the theories on which Dr. Nelson is working are spontaneous violation of CP (charge conjugation and parity symmetry), which may explain the origin of the asymmetry observed between matter and antimatter; the Little Higgs theory, which may explain why the Higgs boson must be relatively light; and the theory of "accelerons," which relates neutrino masses to the cosmological dark energy responsible for the relatively recent acceleration of the expansion of the Universe. Dr. Nelson received a Guggenheim Fellowship in 2004.

Rene A. Ong is a professor of physics and astronomy at the University of California at Los Angeles. He is an experimental and observational astrophysicist working in gamma-ray astronomy and cosmic ray physics, using both ground-based and spaceborne instruments. He is also involved in the search for dark matter using indirect detection methods. Dr. Ong is currently the spokesperson for the Veritas gamma-ray telescope array. He was an A.P. Sloan Foundation Fellow and is a Fellow of the American Physical Society. He participated in two panels of the 2000 NRC Decadal Survey in Astronomy and Astrophysics and served as a member of the NRC Neutrino Facilities Assessment Committee and the recently completed Particle Astrophysics and Gravitation Panel for the Astronomy 2010 survey. Dr. Ong has been a member of numerous advisory committees for funding agencies and national laboratories, including the Astronomy and Astrophysics Advisory Committee, the High-Energy Physics Advisory Panel (HEPAP), the Experimental Program Advisory Committee for SLAC, and the Conseil Scientifique for the Laboratoire Leprince Ringuet of the Ecole Polytechnique. He chaired the Scientific Assessment Group for Experiments in Non-Accelerator Physics, a HEPAP subpanel, in 2004. Dr. Ong serves as a member of the Scientific Advisory Committee of the European AStroParticle ERAnet (ASPERA) group. 
Frank J. Sciulli (NAS) is the Pupin Professor of Physics Emeritus at Columbia University. He is an experimental physicist with a broad career encompassing measurements of K-mesons corroborating quark selection rules, leading collaborations in measurements of neutrino interactions with nucleons that demonstrated the existence of neutral weak currents and the quark-gluon constituency of nucleons; and leading the U.S. effort on the ZEUS program involving colliding beam experiments with high-energy electrons and protons. He is a member of the National Academy of Sciences, a fellow of the American Physical Society, and a fellow of the American Association for the Advancement of Science. He was the recipient of the Humboldt Foundation Research Award and of the American Physical Society W.K.H. Panofsky Prize. He has served as chair of the Columbia Physics Department, has chaired two subpanels of the High Energy Physics Advisory Panel and served on a third, chaired the Science Policy Committee at SLAC National Accelerator Laboratory, and served on the Extended Science Council at the Deutsches Elektronen-Synchrotron. He has also served on two NRC committees: Neutrinos and Beyond and Connecting Quarks with the Cosmos. He was recently co-chair of the Sanford Laboratory Program Advisory Committee and is presently on the board of directors for the Fermi Research Alliance, the management organization for Fermilab, and chairs its Physics Committee.

Marjorie Shapiro is a senior faculty member in the Physics Division at the Lawrence Berkeley National Laboratory and a professor of physics at the University of California at Berkeley. She is an experimental physicist with broad expertise in particle physics. She is a member of the ATLAS collaboration at CERN and is a former collaborator on the Collider Detector at Fermilab. Dr. Shapiro served as a member of the P5 Panel for the Prioritrization of Particle Physics Projects for DOE and NSF in 2003 and 2004 and in 2008, the HEPAP Subpanel on Planning for the Future of U.S. High Energy Physics in 1997 and 1998, the NSF Special Emphasis Panel on Experimental Particle Physics in 1995, the NSF Review Panel on Particle Physics in 1996, and the NRC Committee on Experimental Particle Physics from 1995 to 1997. She served on the CESR Program Advisory Committee (1994-1996), the SLAC Scientific Policy Committee (1995-1998), HEPAP (1997-2000), and the Fermilab Research Associates Visiting Committee for Fermilab Scientific Programs (2009-2011). She has served on numerous visiting committees to physics departments, including Harvard, Toronto, Carnegie-Mellon, University of California at Santa Barbara, UCLA, and the University of California at Riverside and was a member of the external review committee for the Harvard University Planning Committee on Science and Engineering Report on the Future of Science at Harvard in 2006. Dr. Shapiro is a fellow of the American Physical Society. She served as chair of the Berkeley Physics Department from June 2004 through June 2007. 
James M. Tiedje (NAS) is University Distinguished Professor of Microbiology and Molecular Genetics and of Crop and Soil Sciences at Michigan State University and is director of the Center for Microbial Ecology, one of the original NSF-funded Science and Technology Centers. He is a distinguished microbial ecologist with important contributions to denitrification, bioremediation, and molecular ecology. Recently he made notable contributions using genomics and metagenomics to the understanding of ecological functions, speciation, and niche adaptation. He has served as editor in chief of Applied and Environmental Microbiology and editor of Microbial and Molecular Biology Reviews. He served on the NRC Board on Life Sciences, chaired EPA's Science Advisory Panel, and serves on DOE's Biological and Environmental Research Advisory Committee. He was president of the American Society for Microbiology and of the International Society of Microbial Ecology. He shared the 1992 Finley Prize from UNESCO for internationally significant research contributions in microbiology and was recently awarded an Einstein Professorship by the Chinese Academy of Sciences. He is a fellow of the AAAS, the American Academy of Microbiology, and the Soil Science Society of America.

David Wark is a professor of physics at Imperial College London and a senior laboratory fellow at the STFC Rutherford Appleton Laboratory. He is an internationally renowned authority on neutrino physics, recognized for his work on a series of groundbreaking neutrino experiments, including the SAGE solar neutrino experiment, which first showed that there was a deficit of neutrinos from the protonproton solar cycle, and, more recently, on the Sudbury Neutrino Observatory in Canada, where he served as U.K. co-spokesman. Currently, Dr. Wark is one of the principal investigators and international spokesmen for the neutrino oscillation experiment being constructed in Japan. He is a member of the Scientific Policy Committee at CERN and was formerly a member of the CERN SPSC committee, which supervised the CNGS long-baseline neutrino oscillation program, and was a founding member of the European Committee for Coordination of Astroparticle Physics. Dr. Wark was the first chair of the U.K.'s Astroparticle Physics Advisory Panel, a former member of the European Committee for Future Accelerators, was the Chair of the European Physical Society High Energy Particle Physics Division, and has served on the advisory committees for SNOLAB and the CanFranc underground laboratory. He was awarded the Institute of Physics Rutherford Prize in 2004 for contributions to astroparticle physics, is a former President of the Physics Section of the British Association for the Advancement of Science, and is a Fellow of the Royal Society and of the Institute of Physics. 


\section{$\mathrm{D}$ Survey of the Principal Underground Laboratories}

Existing underground facilities are scattered throughout the world. The principal ones are discussed in this section in geographical order, from west to east, starting from Europe. In developing this material, the committee drew from the results of two recent comprehensive surveys of underground laboratories. ${ }^{1}$

\section{EUROPE}

\section{Boulby Underground Laboratory (U.K.)}

http://www.hep.shef.ac.uk/research/dm/boulby/boulby.php

Boulby Underground Laboratory was developed as an underground laboratory in 1988 in an active potash mine on the northeast coast of England.

- Its principal research space is at a depth of $1,000 \mathrm{~m}$.

- Access is through a vertical shaft, and the salt environment of the surrounding rock limits the cavities' width and height to about $5 \mathrm{~m}$. A clean area of

\footnotetext{
${ }^{1}$ A. Bettini. 2011. Underground laboratories. Nuclear Instruments and Methods in Physics Research Section A: Accelerators, Spectrometers, Detectors and Associated Equipment 626: S64-S68. E. Coccia. 2010. Underground laboratories: Cosmic silence, loud science. Journal of Physics Conference Series 203: 012023.
} 
approximately $1,500 \mathrm{~m}^{2}$ is available for experiments. There is potential for expansion.

- The flux of neutrons with energies greater than $0.5 \mathrm{MeV}$ is $1.7 \times 10^{-2}$ $\mathrm{m}^{-2} \mathrm{~s}^{-1}$; the muon flux is $4.5 \times 10^{-2} \mathrm{~m}^{-2} \mathrm{~s}^{-1}$.

- A building on the surface $\left(200 \mathrm{~m}^{2}\right)$ hosts laboratories for computing, electronics, and chemistry, offices, a conference room, changing rooms, mess rooms, a mechanical workshop, and storage and construction rooms. About 30 scientists work at the laboratory.

- The scientific program is focused on dark matter search: ZEPLIN II and ZEPLIN III, both based on two phases of Xe, and DRIFT II, which is in the research and development phase. In addition to the physics program, there are low radioactivity measurements and geophysics research.

\section{Laboratorio Subterráneo de Canfranc (Spain) \\ http://www.lsc-canfranc.es/}

The underground laboratory at Canfranc, Spain, was created beneath the Pyrenees mountains in the 1980s by the Nuclear and High-Energy Physics Department of Saragossa University and expanded in 2005, after the excavation of a road tunnel. It is managed by a consortium of the Ministry for Education and Science, the Government of Aragon, and the University of Saragossa.

- The maximum rock coverage is $850 \mathrm{~m}$.

- The access is horizontal, via a road tunnel.

- The available underground space consists of Hall A, measuring $40 \times 15 \times$ $12(\mathrm{~h}) \mathrm{m}^{3}$, Hall B, measuring $15 \times 10 \times 8(\mathrm{~h}) \mathrm{m}^{3}$, a clean room of $45 \mathrm{~m}^{2}$, and service facilities in a $215 \mathrm{~m}^{2}$ space.

- The muon flux is between $2 \times 10^{-3}$ and $4 \times 10^{-3} \mathrm{~m}^{-2} \mathrm{~s}^{-1}$, depending upon the location, while the neutron flux is $2 \times 10^{-2} \mathrm{~m}^{-2} \mathrm{~s}^{-1}$. The Rn activity in the air is $50-80 \mathrm{~Bq} / \mathrm{m}^{3}$ with a ventilation of $11,000 \mathrm{~m}^{3} / \mathrm{h}$ - that is, one lab volume in $40 \mathrm{~min}$.

- The surface building contains headquarters, administration, a library, a meeting room, offices, laboratories, storage and a mechanical workshop, safety structures, and management, for a total of approximately $1,500 \mathrm{~m}^{2}$.

- The scientific program, developed with the advice of an international scientific committee, includes the following experiments: on dark matter searches, ANAIS (looking for an annual modulation with NaI crystals) and ROSEBUD (developing scintillation bolometers in the frame of Eureka), and on double-beta decay, NEXT (with $100 \mathrm{~kg}$ of enriched Xe). Two other projects are ancillary to experiments in other laboratories: BiPo for the 
neutrinoless double-beta decay project SuperNEMO and SUPERKGD (SK) for material screening in view of a possible addition of Gd into the SK water. A new hall to host an underground accelerator facility dedicated to nuclear astrophysics is under design. A special facility is dedicated to other low-radioactivity measurements.

\section{Laboratoire Souterrain de Modane (France) \\ http://www-lsm.in2p3.fr/}

The Laboratoire Souterrain de Modane (LSM) is operated jointly by the Institut National de Physique Nucléaire et de Physique des Particules (CNRS/IN2P3) and the Commissariat à l'Energie Atomique/Direction des Sciences de la Matière (CEA/DSM) of the Centre National de la Recherche Scientifique. The excavation of the Laboratory started in 1979 and was completed in 1982.

- The vertical direction of the rock measures $1,700 \mathrm{~m}$.

- Horizontal access is provided through a connection to the Fréjus roadway tunnel.

- The main hall volume is $30 \times 10 \times 11(\mathrm{~h}) \mathrm{m}^{3}$, the gamma hall has an area of 70 $\mathrm{m}^{2}$, and two smaller halls have $18 \mathrm{~m}^{2}$ and $21 \mathrm{~m}^{2}$ areas, for a total of $400 \mathrm{~m}^{2}$.

- The muon flux is $4.7 \times 10^{-5} \mathrm{~m}^{-2} \mathrm{~s}^{-1}$. The neutron flux is $5.6 \times 10^{-2} \mathrm{~m}^{-2} \mathrm{~s}^{-1}$. Low radon activity in the air, $15 \mathrm{~Bq} / \mathrm{m}^{3}$, is obtained by intaking fresh air at the rate of 1.5 lab volumes per hour. An "antiradon factory" produces 150 $\mathrm{m}^{3}$ per hour of air with $10 \mathrm{mBq} / \mathrm{m}^{3}$.

- A surface building hosts outreach facilities, offices, technical laboratories, and sleeping rooms. The user community is about 200, with scientists from 31 institutions in seven countries. On-site support personnel typically consist of eight technicians and engineers and one postdoc.

- The scientific program, developed with the advice of an international scientific committee, includes the following experiments: NEMO 3 (doublebeta decay), EDELWEISS (dark matter), and a low-radioactivity counting facility.

- A $60,000 \mathrm{~m}^{3}$ extension of the lab has been proposed (Ulisse project) to take advantage of the opportunity presented by the construction of a new tunnel approved by the French and Italian governments to increase the safety conditions of traffic in the tunnel. Two large halls are foreseen: hall A, $24 \times$ $100 \mathrm{~m}^{2}$, and hall B, $18 \times 50 \mathrm{~m}^{2}$. An extremely low background environment will be obtained in hall $\mathrm{B}$ by surrounding its central volume with a water shield and by artificially producing an atmosphere with very low $\mathrm{Rn}$ content $\left(0.1 \mathrm{mBq} / \mathrm{m}^{3}\right)$. 


\section{Laboratori Nazionali del Gran Sasso (Italy) \\ http://www.lngs.infn.it/}

The Laboratori Nazionali del Gran Sasso (LNGS) is a national laboratory of Italy's Istituto Nazionale di Fisica Nucleare (INFN). The construction started in 1982 and was completed by 1987.

- The vertical rock overburden is $1,400 \mathrm{~m}$.

- Access is horizontal, through a freeway.

- The underground laboratory consists of three main halls, A, B and C, each with dimensions of about $100 \times 20 \times 18(\mathrm{~h}) \mathrm{m}^{3}$, plus ancillary tunnels that provide space for services and small-scale experiments. The total area is $17,300 \mathrm{~m}^{2}$, and the total volume $180,000 \mathrm{~m}^{3}$.

- Muon flux is $3 \times 10^{-4} \mathrm{~m}^{-2} \mathrm{~s}^{-1}$; neutron flux is $3.78 \times 10^{-2} \mathrm{~m}^{-2} \mathrm{~s}^{-1}$, and measured radon in the air is $50-120 \mathrm{~Bq} / \mathrm{m}^{3}$. The ventilation system provides one lab volume of fresh air every $3.5 \mathrm{~h}$.

- Services hosted on the surface campus include offices, a mechanical workshop, storage facilities, a chemical laboratory, an electronic workshop, an assembly hall, computer and networking facilities, a library, a canteen, sleeping quarters, conference rooms, and headquarters for administration.

- The scientific user community consists of 752 scientists from 26 countries. Personnel (physicists, engineers, technicians, administration) include a permanent staff of 76 and about 20 nonpermanent positions.

- LNGS is operated as an international laboratory. An international scientific committee, appointed by INFN, advises the director. The rich experimental program includes the CERN to Gran Sasso neutrino beam experiments OPERA and ICARUS; dark matter search, with LIBRA, CRESST2, XENON, and WARP; neutrinoless double-beta decay, with COBRA, CUORE, and GERDA; solar neutrinos (and geoneutrinos) experiments with BOREXINO; supernova neutrinos studies with LVD; and nuclear astrophysics experiments with LUNA2. A special facility is dedicated to low-radioactivity measurements. The laboratory also supports several experiments on geology, biology, and environmental issues. Two 90-m-long tunnels were built for two Michelson interferometers for geology studies.

\section{Centre for Underground Physics in Pyhäsalmi (Finland) http://cupp.oulu.fi/}

- Hosted in a working mine. Access is both via shaft and via an inclined tunnel. 
- Several cavities that are no longer being mined are available for use as laboratory space at different depths down to $980 \mathrm{~m}$, for a total area of more than $1,000 \mathrm{~m}^{2}$. Presently the mine works at depths between $1,000 \mathrm{~m}$ and $1,400 \mathrm{~m}$.

- The principal experiment on-site is the EMMA experiment, an array of cosmic ray detectors currently being installed $75 \mathrm{~m}$ underground and designed to study energetic cosmic rays. Small lab and office space is available in a surface building and a guesthouse is also available.

- The personnel consist of about three people on-site and three in Oulu University.

\section{Solotvina Underground Laboratory (Ukraine) http://lpd.kinr.kiev.ua/LPD_SUL.htm}

This laboratory was constructed in a salt mine in 1984 by the Lepton Physics Department (LPD) of the Institute for Nuclear Research, under the Ukrainian National Academy of Sciences.

- The lab is $430 \mathrm{~m}$ deep in salt $(\approx 1,000 \mathrm{~m}$.w.e. $)$.

- Access is vertical by the mine cage and depends on the timetable of the mine.

- The laboratory space is divided into a main hall, $25 \times 18 \times 8(\mathrm{~h}) \mathrm{m}^{3}$, and four chambers $6 \times 6 \times 3(\mathrm{~h}) \mathrm{m}^{3}$. The total area is approximately $1,000 \mathrm{~m}^{2}$.

- The muon flux is $1.7 \times 10^{-2} \mathrm{~m}^{-2} \mathrm{~s}^{-1}$. The neutron flux is $2.7 \times 10^{-2} \mathrm{~m}^{-2} \mathrm{~s}^{-1}$. Radon concentration in air is $33 \mathrm{~Bq} / \mathrm{m}^{3}$.

- On the surface, three living rooms are available for visiting researchers. Staff consists of 14 technicians and engineers. Typically, about 11 researchers and Ph.D. students from LPD work in the laboratory.

- The main subject of the scientific program is on double-beta decay, preparing a new ${ }^{116} \mathrm{Cd}$ experiment using $1-2 \mathrm{~kg}{ }^{116} \mathrm{CdWO}_{4}$ higher quality crystal scintillators and developing R\&D projects on scintillators and for the SuperNEMO project.

\section{Baksan Neutrino Observatory (Russia) http://www.inr.ac.ru/INR/}

The Baksan Neutrino Observatory laboratory is operated by the Institute for Nuclear Research of the Russian Academy of Sciences. It is the oldest underground facility in the world built specifically for scientific research. It is placed under Mount Andyrchi in the Caucasus. 
- The access is horizontal via two dedicated tunnels, with train transportation.

- In this lab the muon flux is $3 \times 10^{-5} \mathrm{~m}^{-2} \mathrm{~s}^{-1}$. The neutron flux (with energies greater than $1 \mathrm{MeV}$ ) is $1.4 \times 10^{-3} \mathrm{~m}^{-2} \mathrm{~s}^{-1}$. The $\mathrm{Rn}$ activity is $40 \mathrm{~Bq} / \mathrm{m}^{3}$ with a fresh air input of $60,000 \mathrm{~m}^{3} / \mathrm{h}$.

- Personnel providing all necessary services (heating station, water supply system, first aid medical help, transportation, safety and so on) live in a new village, called Neutrino. The staff who are directly engaged in research number 50 to 60 .

- A large hall, $24 \times 24 \times 16 \mathrm{~m}^{3}$ in volume, $300 \mathrm{~m}$ deep, hosts the Baksan Underground Scintillation Telescope. The telescope has been ready to observe neutrinos from galactic supernovas since 1978. Another hall, 60 $\times 10 \times 12 \mathrm{~m}^{3}$ at a vertical depth of 2,100 m, hosts the Soviet-American Gallium Experiment's gallium germanium neutrino telescope. Low-background chambers with volumes from $100 \mathrm{~m}^{3}$ to $300 \mathrm{~m}^{3}$ are used for R\&D on dark matter and neutrinoless double-beta decay search as well as for gravitational wave search and for some geophysics measurements.

\section{ASIA}

\section{India-Based Neutrino Observatory (India)}

http://www.imsc.res.in/ ino/

The India-based Neutrino Observatory (INO) is a project under development to create an underground laboratory in southern India.

- Two main underground cavities are foreseen: Lab 1 with a volume of $26 \times$ $135 \times 25(\mathrm{~h}) \mathrm{m}^{3}$ and Lab 2 with $53.4 \times 12.5 \times 8.6(\mathrm{~h}) \mathrm{m}^{3}$ plus connection tunnels and services. Access will be horizontal through a dedicated 2-km tunnel. On the surface the facility is planned to have a $1,400 \mathrm{~m}^{2}$ building for administration, offices, shops, and the like; a $2,750 \mathrm{~m}^{2}$ building with lecture hall and guesthouse; and a residential complex with 20 units. A support staff of 50 to 100 is expected.

- The main experiment foreseen for INO is ICAL, a 50-kT magnetized iron tracking calorimeter for atmospheric and very-long-baseline accelerator neutrinos. It will occupy only a fraction of Lab 1 .

\section{Kamioka Observatory (Japan) \\ http://www-sk.icrr.u-tokyo.ac.jp/index-e.html}

The Kamioka Observatory is operated by the Institute for Cosmic Ray Research, University of Tokyo. It was established in 1983. 
- The vertical coverage is $1,000 \mathrm{~m}$.

- Access is horizontal by car, with no interference from mining activity.

- The measured muon flux is $3 \times 10^{-3} \mathrm{~m}^{-2} \mathrm{~s}^{-1}$; thermal neutron flux is $8 \times 10^{-2}$ $\mathrm{m}^{-2} \mathrm{~s}^{1}$, and nonthermal neutron flux is $11 \times 10^{-2} \mathrm{~m}^{-2} \mathrm{~s}^{-1}$. The ventilation is $3,000 \mathrm{~m}^{3} / \mathrm{h}$. Buildings for offices and computer facilities are available on the surface. On-site staff consists of 19 scientists, 3 technical support staff, and 4 administrators. The average number of scientific users is more than 200.

- The underground structures and related scientific activities are as follows:

— Hall SK (50 m diameter) hosting Super-Kamiokande, the largest experiment underground. It is the target of the T2K experiment and is a thirdgeneration neutrino oscillation experiment on an intense off-axis beam of muon neutrinos $\left(v_{\mu}\right)$ produced at the J-PARC accelerator facility 295 $\mathrm{km}$ from the Super-K detector.

- Clean room $\left(10 \times 5 \mathrm{~m}^{2}\right)$ with XMASS prototype;

- Hall 40 (L-shape, $40 \times 4 \mathrm{~m}$ arms) hosting the purification tower for XMASS and the NEWAGE experiment on dark matter;

- Hall 100 (L-shape, $100 \times 4 \mathrm{~m}$ arms) with CLIO, a prototype gravitational antenna (to be terminated in 2013);

— The new Hall A $\left(15 \times 21 \mathrm{~m}^{2}\right)$ hosting XMASS $800 \mathrm{~kg}$;

- The new Hall B $\left(6 \times 11 \mathrm{~m}^{2}\right)$ hosting CANDLE on double-beta decay;

- In the same mountain, the Kamioka liquid scintillator antineutrino detector (KamLAND experiment) is operated by the Neutrino Centre, Tohoku University. It studies neutrino oscillation by measuring antineutrinos from the commercial power reactors surrounding the site; and

- The underground large cryogenic gravitational antenna LCGT, which has baseline lengths of $3 \times 3 \mathrm{~km}$, has been recently approved.

Further enlargements are under development to accommodate more experiments.

\section{China Deep Underground Science and Engineering Laboratory/ China JinPing Deep Underground Laboratory}

Recently, the China Deep Underground Science and Engineering Laboratory (CDUSEL), designed to be the world's deepest, and possibly its largest, underground laboratory, was launched in China. ${ }^{2}$ The facility plans to take advantage of infrastructure being developed by the Ertan Hydropower Development Company (EHDC), which is installing a series of 21 hydroelectric power stations on

\footnotetext{
${ }^{2}$ Qian, Yue. 2010. "Status and prospects of China JinPing Deep Underground Laboratory (CJPL) and China Dark Matter Experiment (CDEX)." Presentation at the TeV Particle Astrophysics 2010 Conference, Paris, France, July.
} 
the Yalong River in central China. A system of tunnels $17.5 \mathrm{~km}$ long will cut a big U-turn in the river, under 4,193-m-tall JinPing mountain.

- The future underground laboratory will have a maximum vertical rock overburden of 2,500 $\mathrm{m}$ and a greater than $1,500 \mathrm{~m}$ overburden in 70 percent of the directions.

- The access will be horizontal, from two sides.

- Two small experimental halls $5 \times 5 \times 30 \mathrm{~m}^{3}$ are under construction. The final size of the laboratory has not been made public, although it has been reported that the laboratory will be designed as an international facility, open to the world community.

- Measurements of the muon flux (expected to be very low, on the order of $20 \mathrm{~m}^{-2} \mathrm{y}^{-1}$ ), the neutron flux and radon concentration in the air will be performed shortly. A working group, including scientists and engineers from major Chinese institutions and universities, as well as EHDC, has been established to further develop plans for this facility.

\section{NORTH AMERICA}

\section{Sudbury Neutrino Observatory Laboratory (Canada)}

http://www.snolab.ca/ also http://www.sno.phy.queensu.ca/

The Sudbury Neutrino Observatory was excavated in the 1990s in an operating nickel mine. The original SNO cavity, a $200 \mathrm{~m}^{2}$ area, is now being freed for further experimental activity. To this original space, new structures have been added to form a new laboratory, the SNOLAB, whose main features are reported here.

- The vertical coverage is 2,000 m under a flat surface.

- The access is vertical, through the shaft of the working mine, and is available daily.

- Space underground consists of a main hall $18 \times 15 \times 15-19.5(\mathrm{~h}) \mathrm{m}^{3}$, a service hall of about $180 \mathrm{~m}^{2}$, and a number of narrow volumes called "ladder labs." The volume for a further structure, called the "cryopit," has also been excavated. This hall is designed to cope with the safety issues surrounding large volumes of cryogenic fluids. The total area is $7,215 \mathrm{~m}^{2}$, of which 3,055 $\mathrm{m}^{2}$ is available for experiments. The total volume is $46,648 \mathrm{~m}^{3}$, of which $29,555 \mathrm{~m}^{3}$ is available for experiments.

- Measured muon flux is $3 \times 10^{-6} \mathrm{~m}^{-2} \mathrm{~s}^{-1}$, thermal neutron flux is $4.7 \times 10^{-2}$ $\mathrm{m}^{-2} \mathrm{~s}^{-1}$, and fast neutron flux is $4.6 \times 10^{-2} \mathrm{~m}^{-2} \mathrm{~s}^{-1}$. The air has a relatively high 
radon count, $120 \mathrm{~Bq} / \mathrm{m}^{3}$. The ventilation in the smaller lab spaces provides 10 air changes per hour, in the larger ones 5 air changes per hour.

- All of the laboratory will be clean, maintained at Class 1500.

- On the surface, a 3,159- $\mathrm{m}^{2}$ building will host a clean room, laboratories, staging and assembly areas, office space for 60 users, meeting rooms, control rooms, an IT server room, an emergency generator, a high-speed network link off-site, both surface and underground high-speed network links, safety structures, and management. Staff will consist of 30 full-time people.

- The scientific program includes the PICASSO experiment, searching for dark matter $(2 \mathrm{~kg})$ using the superheated bubbles technique, which is running. A new instrument for neutrino research, $\mathrm{SNO}+$, is to be hosted in the former SNO cavity and is based on liquid scintillator for low-energy solar neutrinos, geoneutrinos, and double-beta decay, by dissolving ${ }^{150} \mathrm{Nd}$ in the liquid. Dark matter searches include DEAP/CLEAN with noble liquids, which is operating with a prototype, and the installation of superCDMS with bolometers. More letters of intent for possible future experiments are expected to be reviewed by the facility's experimental advisory committee.

\section{Soudan Underground Laboratory (United States) http://www.soudan.umn.edu/}

The underground structure is hosted in the Soudan Underground Mine state park. The laboratory coexists with a historic state park, which offers mine tours to the public and to school groups. There is no active mining.

- The vertical overburden is $700 \mathrm{~m}$ of rock.

- The access is vertical via a two-compartment, slightly angled shaft. Diameters in excess of $1 \mathrm{~m}$ and lengths in excess of $10 \mathrm{~m}$ pose a problem. Access outside normal park operating hours is possible.

- The muon flux is $2 \times 10^{-3} \mathrm{~m}^{-2} \mathrm{~s}^{-1}$. The neutron interaction rates are approximately $10 \mathrm{~kg}^{-1} \mathrm{~d}^{-1}$ (from low-energy uranium and thorium) or $0.01 \mathrm{~kg}^{-1} \mathrm{~d}^{-1}$ (muons generated in the rock). The radon concentration is seasonal, varying from $300 \mathrm{~Bq} / \mathrm{m}^{3}$ in the winter to $700 \mathrm{~Bq} / \mathrm{m}^{3}$ in the summer. The mine has natural ventilation, about $550 \mathrm{~m}^{3} / \mathrm{h}$ at the laboratories' level. This results in a complete air change every 110 minutes.

- The main facility on the surface is a building of approximately $650 \mathrm{~m}^{2}$ with offices, kitchen, and sanitary facilities. The laboratory has a staff of nine, including secretarial and accounting assistance and network and computer maintenance personnel. There are currently 265 scientific users of the facility. 
- The scientific facility includes the Soudan lab $\left(20 \times 7 \times 10(\mathrm{~h}) \mathrm{m}^{3}\right)$, which hosts the dark matter experiment CDMSII and a low-background counting facility that currently occupies $5 \times 5 \times 3 \mathrm{~m}^{3}$ and will expand to $25 \times 14 \times$ 14(h) $\mathrm{m}^{3}$, if funded; and the MINOS lab, which occupies $35 \times 16 \times 14(\mathrm{~h})$ $\mathrm{m}^{3}$ and is expected to run a few years more with a 2-year decommissioning period at the conclusion, and a high-purity copper fabrication facility that occupies $4 \times 6 \times 3(\mathrm{~h}) \mathrm{m}^{3}$.

\section{Special-Purposes Laboratories (United States)}

Two special-purpose underground research laboratories have been developed in the United States. The Waste Isolation Pilot Plant (WIPP) in bedded salt, at Carlsbad, New Mexico, evolved from an underground laboratory to a full-scale facility and has been used since 1998 as a permanent disposal repository for intermediate (long-lived) radioactive waste. An underground laboratory at Yucca Mountain, Nevada, in volcanic tuff at a depth of $300 \mathrm{~m}$, was developed and used for research into high-level waste disposal until 2010, when a plan for the construction of a permanent repository at the site was submitted to the U.S. Nuclear Regulatory Commission. As with WIPP, the intent is to develop a permanent repository at the site. 
Copyright (C) National Academy of Sciences. All rights reserved. 